\title{
Sunrise integrals with two internal masses and pseudo-threshold kinematics in terms of elliptic polylogarithms
}

\author{
Lijsbeth G.J. Campert, ${ }^{a}$ Francesco Moriello ${ }^{a}$ and Anatoly Kotikov ${ }^{b}$ \\ ${ }^{a}$ ETH Zürich, Institut für theoretische Physik, \\ Wolfgang-Pauli str. 27, 8093, Zürich, Switzerland \\ ${ }^{b}$ Bogolyubov Laboratory for Theoretical Physics, JINR, \\ 141980 Dubna (Moscow region), Russia \\ E-mail: annacampert@gmail.com, francescomoriello@gmail.com, \\ kotikov@theor.jinr.ru
}

ABSTRACT: We consider a set of two-loop sunrise master integrals with two different internal masses at pseudo-threshold kinematics and we solve it in terms of elliptic polylogarithms to all orders of the dimensional regulator.

KEYwords: Perturbative QCD, Scattering Amplitudes

ArXiv EPRINT: 2011.01904 


\section{Contents}

1 Introduction 1

2 The sunrise integral 3

3 Integral representations 3

4 All orders result in terms of elliptic polylogarithms $\quad 6$

4.1 Elliptic polylogarithms 6

4.2 Regularisation 8

4.3 Elliptic polylogarithms and all orders result 9

$\begin{array}{llr}4.4 \text { Sunsets } & 12\end{array}$

$\begin{array}{lll}\text { 4.4.1 } & J_{1,2,2} & 12\end{array}$

$\begin{array}{lll}\text { 4.4.2 } & J_{1,1,1} \text { and } J_{1,1,2} & 13\end{array}$

$\begin{array}{lll}4.5 & \text { Example } & 15\end{array}$

$\begin{array}{llr}5 & \text { Conclusions } & 17\end{array}$

$\begin{array}{ll}\text { A Integral representations for the hypergeometric functions } & 17\end{array}$

B Relations between integrals $J_{2}^{(j)}(q)$ and $J^{(j)}(q)(j=1,3) \quad 23$

$\begin{array}{ll}\text { C Improved representations } & 26\end{array}$

D Leading terms of the $\varepsilon$-expansion and one-fold integrals 32

$\begin{array}{ll}\text { E Definitions for elliptic polylogarithms } & 34\end{array}$

\section{Introduction}

In the last decades much progress has been made in the understanding of the mathematical properties of Feynman integrals. Arguably many of the breakthroughs in this line of research originated from the identification of classes of special functions suited for the solution of Feynman integrals by means of various analytic methods. It is a well-known fact that while many Feynman integrals admit representations in terms of so-called multiple polylogarithms (MPLs) [1,2], this space of functions is not sufficient to express integrals when the number of physical scales is sufficiently large. More recently, the scientific community has centered its attention to the study of Feynman integrals whose geometric properties are defined by elliptic curves. Following early investigations of [3] and [4], many integrals involving elliptic curves have been computed in the literature [5-67].

In a parallel line of research, a class of functions, the so-called Elliptic Multiple Polylogarithms, describing all iterated integrals on the torus has been identified in the mathematics 
literature [68] (see also [69, 70]). While these functions formally solve the problem of generalising MPLs to more complicated geometries, their definition is not naturally suited for physical applications. Progress in this direction has been made in [35], where eMPLs are defined on the complex plane, and their structure naturally adapts to representations of Feynman integrals commonly used in the physics literature (e.g. Feynman parameters).

Special functions such as MPLs and eMPLs, frequently appear when computing Feynman integrals in dimensional regularisation. More specifically, Feynman integrals admit a Laurent expansion with respect to the dimensional regulator and the coefficients of this expansion can be often computed explicitly in terms of known special functions. In practice it is often possible to truncate the Laurent series, as the computation of physically relevant quantities requires only a few expansion orders. Nonetheless it is interesting to explore the analytic structure of these coefficients at higher orders or, more generally, to all orders of the dimensional regulator. In this context, all orders results for the equal- and different-mass sunrise integrals have been obtained in [16] and [55], respectively, in terms of iterated integrals of modular forms.

In this paper we consider a two-loop sunrise integral topology with two internal masses and pseudo-threshold kinematics [71] (see also $[6,56]$ ). More precisely, we consider two different internal masses, denoted by $m$ and $M$, and external kinematics $p^{2}=-m^{2}$. This integral family appears when considering non-relativistic limits of Quantum Chromodynamics (NRQCD) and Quantum Electrodynamics (NRQED) (see for example [6] and [56]). Examples of phenomenological applications of NRQCD are the study of heavy-quarkonium production and decay (see refs. [72-77] and references therein) and the near-threshold production of $t \bar{t}[40,41,78-94]$. Similarly, an important application of NRQED is the calculation of the parapositronium decay rate (see refs. [95-101]).

The analytic structure of the sunrise topology considered in this paper has been studied by means of differential equations in [102-106] and by using an effective-mass analysis in [107-110]. This integral family admits a closed-form solution in terms of ${ }_{4} F_{3}$ hypergeometric functions, as shown in [71] (the corresponding off-shell diagrams with equal masses are considerably more complicated and their explicit solution requires Appell's $F_{2}$ hypergeometric functions [111]). Moreover, similar results exist for three-point and fourpoint two-loop Feynman diagrams in NRQCD kinematics (see ref. [56] for $O\left(\varepsilon^{0}\right)$ results and [112] for results to every order in $\varepsilon$ in terms of ${ }_{s+1} F_{s}$-hypergeometric functions with $s \leq 7)$. In this paper we consider the two-loop sunrise integral family discussed above and derive results in terms of eMPLs valid to all orders of the dimensional regulator.

The paper is organised as follows. In section 2 we define the sunrise integral family and we present the ${ }_{4} F_{3}$-hypergeometric representation for the finite sunrise integral considered in this paper. In section 3 we present a double integral representation for the relevant ${ }_{4} F_{3^{-}}$ hypergeometric functions. In section 4 we review properties of eMPLs and we present our all orders result for the sunrise integral. In section 5 we draw our conclusions. In appendix A, $\mathrm{B}$ and $\mathrm{C}$ we present the detailed derivations of the double integral representations for the relevant ${ }_{4} F_{3}$-hypergeometric functions. In appendix $\mathrm{D}$ we provide a representation for the integral family in terms of one-dimensional integrals over polylogarithmic expressions, up to $O(\varepsilon)$. In appendix E we provide definitions relevant to the main results of the paper. 


\section{The sunrise integral}

Following ref. [71] we study the sunrise integral topology defined as,

$$
J_{i_{1}, i_{2}, i_{3}}\left(m^{2}, M^{2}\right)=\left.\iint \frac{d^{D} k_{1} d^{D} k_{2}\left(\mu^{2}\right)^{2 \varepsilon}}{\left[k_{2}^{2}-m^{2}\right]^{i_{1}}\left[k_{1}^{2}-M^{2}\right]^{i_{2}}\left[\left(k_{1}-k_{2}-q\right)^{2}-M^{2}\right]^{i_{3}}}\right|_{q^{2}=-m^{2}},
$$

with $D=4-2 \epsilon$. This integral family has three master integrals, which can be chosen to be $J_{1,1,1}, J_{1,1,2}, J_{1,2,2}$ and which can be solved in closed form in terms of hypergeometric functions [71] as,

$$
\begin{aligned}
& J_{1,2,2}\left(m^{2}, M^{2}\right)=-\frac{\hat{N}_{1}}{M^{2}} \frac{(1+\epsilon)}{\epsilon(1-\epsilon)} \times\left[\frac{1}{6}{ }_{4} F_{3}\left(\begin{array}{c}
1, \frac{3}{2}, 1+\frac{\epsilon}{2}, \frac{3}{2}+\frac{\epsilon}{2} \\
2-\epsilon, \frac{5}{4}, \frac{7}{4}
\end{array} \mid-\frac{m^{4}}{4 M^{4}}\right)\right. \\
& -\left(\frac{M^{2}}{m^{2}}\right)^{1-\epsilon} \frac{\epsilon}{(1+\epsilon)(1+2 \epsilon)}{ }^{4} F_{3}\left(\begin{array}{c}
1, \frac{1}{2}+\epsilon, 1+\frac{\epsilon}{2}, 1+\epsilon \\
\frac{3}{2}-\frac{\epsilon}{2}, \frac{3}{4}+\frac{\epsilon}{2}, \frac{5}{4}+\frac{\epsilon}{2}
\end{array} \mid-\frac{m^{4}}{4 M^{4}}\right) \\
& \left.-\left(\frac{M^{2}}{m^{2}}\right)^{-\epsilon} \frac{1-\epsilon}{(2-\epsilon)(3+2 \epsilon)}{ }_{4} F_{3}\left(\begin{array}{c}
1, \frac{3}{2}+\frac{\epsilon}{2}, 1+\epsilon, \frac{3}{2}+\epsilon \\
2-\frac{\epsilon}{2}, \frac{5}{4}+\frac{\epsilon}{2}, \frac{7}{4}+\frac{\epsilon}{2}
\end{array} \mid-\frac{m^{4}}{4 M^{4}}\right)\right] \text {, } \\
& J_{1,1,2}\left(m^{2}, M^{2}\right)=N_{1} \frac{1}{2 \epsilon(1-\epsilon)} \times\left[{ }_{4} F_{3}\left(\begin{array}{c}
1, \frac{1}{2}, 1+\frac{\epsilon}{2}, \frac{1}{2}+\frac{\epsilon}{2} \\
2-\epsilon, \frac{3}{4}, \frac{5}{4}
\end{array} \mid-\frac{m^{4}}{4 M^{4}}\right)\right. \\
& -\left(\frac{M^{2}}{m^{2}}\right)^{1-\epsilon} \frac{1}{\epsilon}{ }_{4} F_{3}\left(\begin{array}{c}
1, \frac{1}{2}+\epsilon, \frac{\epsilon}{2}, \epsilon \\
\frac{3}{2}-\frac{\epsilon}{2}, \frac{1}{4}+\frac{\epsilon}{2}, \frac{3}{4}+\frac{\epsilon}{2}
\end{array} \mid-\frac{m^{4}}{4 M^{4}}\right) \\
& \left.-2\left(\frac{M^{2}}{m^{2}}\right)^{-\epsilon} \frac{1-\epsilon}{(2-\epsilon)(1+2 \epsilon)}{ }_{4} F_{3}\left(\begin{array}{c}
1, \frac{1}{2}+\frac{\epsilon}{2}, 1+\epsilon, \frac{1}{2}+\epsilon \\
2-\frac{\epsilon}{2}, \frac{3}{4}+\frac{\epsilon}{2}, \frac{5}{4}+\frac{\epsilon}{2}
\end{array} \mid-\frac{m^{4}}{4 M^{4}}\right)\right], \\
& J_{1,1,1}\left(m^{2}, M^{2}\right)=-M^{2} \hat{N}_{1} \frac{1}{\epsilon^{2}(1-\epsilon)} \times\left[{ }_{4} F_{3}\left(\begin{array}{c}
1, \frac{1}{2}, \frac{\epsilon}{2}, \frac{1}{2}+\frac{\epsilon}{2} \\
2-\epsilon, \frac{3}{4}, \frac{5}{4}
\end{array} \mid-\frac{m^{4}}{4 M^{4}}\right)\right. \\
& +\left(\frac{M^{2}}{m^{2}}\right)^{1-\epsilon} \frac{1}{(1-2 \epsilon)}{ }_{4} F_{3}\left(\begin{array}{c}
1,-\frac{1}{2}+\epsilon, \frac{\epsilon}{2}, \epsilon \\
\frac{3}{2}-\frac{\epsilon}{2}, \frac{1}{4}+\frac{\epsilon}{2}, \frac{3}{4}+\frac{\epsilon}{2}
\end{array} \mid-\frac{m^{4}}{4 M^{4}}\right) \\
& \left.-\left(\frac{M^{2}}{m^{2}}\right)^{-\epsilon} \frac{1-\epsilon}{(2-\epsilon)(1+2 \epsilon)}{ }_{4} F_{3}\left(\begin{array}{c}
1, \frac{1}{2}+\frac{\epsilon}{2}, \epsilon, \frac{1}{2}+\epsilon \\
2-\frac{\epsilon}{2}, \frac{3}{4}+\frac{\epsilon}{2}, \frac{5}{4}+\frac{\epsilon}{2}
\end{array} \mid-\frac{m^{4}}{4 M^{4}}\right)\right] \text {, }
\end{aligned}
$$

where the normalization constant is,

$$
\hat{N}_{1}=\frac{\Gamma^{2}(1+\epsilon)\left(\mu^{2}\right)^{2 \epsilon}}{\left(m^{2} M^{2}\right)^{\epsilon}} \frac{m^{2}}{M^{2}}
$$

\section{Integral representations}

In this section we show that the hypergeometric functions of eqs. (2.2)-(2.4) admit a twofold integral representation. The derivation of this result relies on multiple identities for hypergeometric functions $[6,56]$ and is presented in appendix A. This representation will be used in the next sections to derive an explicit expression for the sunrise integrals considered in this paper valid to all orders of the dimensional regulator in terms of eMPLs. 
We introduce the dimensionless ratio,

$$
t=\frac{m^{2}}{2 M^{2}},
$$

and restrict ourselves to the Euclidean region $t \in[0,1]$. We find,

$$
\begin{aligned}
& J_{1,2,2}=\frac{\hat{N}_{1}}{M^{2}}\left[J_{1,2,2}^{(1)}(t)+(2 t)^{\varepsilon-1} J_{1,2,2}^{(2)}(t)+(2 t)^{\varepsilon} J_{1,2,2}^{(3)}(t)\right], \\
& J_{1,1,2}=\hat{N}_{1}\left[J_{1,1,2}^{(1)}(t)+(2 t)^{\varepsilon-1} J_{1,1,2}^{(2)}(t)+(2 t)^{\varepsilon} J_{1,1,2}^{(3)}(t)\right], \\
& J_{1,1,1}=M^{2} \hat{N}_{1}\left[J_{1,1,2}^{(1)}(t)+(2 t)^{\varepsilon-1} J_{1,1,2}^{(2)}(t)+(2 t)^{\varepsilon} J_{1,1,2}^{(3)}(t)\right],
\end{aligned}
$$

where,

$$
\begin{aligned}
& J_{1,2,2}^{(1)}(t)=-\frac{1+\varepsilon}{6 \varepsilon(1-\varepsilon)}{ }_{4} F_{3}\left(\begin{array}{c}
1, \frac{3}{2}, 1+\frac{\epsilon}{2}, \frac{3}{2}+\frac{\epsilon}{2} \\
2-\epsilon, \frac{5}{4}, \frac{7}{4}
\end{array} \mid-t^{2}\right)=\frac{\hat{K}}{2^{2+2 \epsilon} \varepsilon t^{2}} I_{1}^{(1)}(t), \\
& J_{1,1,2}^{(1)}(t)=\frac{1}{2 \varepsilon(1-\varepsilon)}{ }_{4} F_{3}\left(\begin{array}{c}
1, \frac{1}{2}, 1+\frac{\epsilon}{2}, \frac{1}{2}+\frac{\epsilon}{2} \\
2-\epsilon, \frac{3}{4}, \frac{5}{4}
\end{array} \mid-t^{2}\right)=\frac{\hat{K}}{2^{1+2 \epsilon}(1-2 \varepsilon) \varepsilon t^{2}} I_{2}^{(1)}(t), \\
& J_{1,1,1}^{(1)}(t)=-\frac{1}{\varepsilon^{2}(1-\varepsilon)}{ }_{4} F_{3}\left(\begin{array}{c}
1, \frac{1}{2}, \frac{\epsilon}{2}, \frac{1}{2}+\frac{\epsilon}{2} \\
2-\epsilon, \frac{3}{4}, \frac{5}{4}
\end{array} \mid-t^{2}\right)=-\frac{1}{\varepsilon^{2}(1-\varepsilon)} \\
& -\frac{\hat{K}}{2^{2 \epsilon-1} \varepsilon(1-2 \varepsilon)^{2} t^{2}} \tilde{I}_{3}^{(1)}(t) \text {, } \\
& J_{1,2,2}^{(2)}(t)=\frac{1}{(1+2 \varepsilon)(1-\varepsilon)}{ }_{4} F_{3}\left(\begin{array}{c}
1, \frac{1}{2}+\epsilon, 1+\frac{\epsilon}{2}, 1+\epsilon \\
\frac{3}{2}-\frac{\epsilon}{2}, \frac{3}{4}+\frac{\epsilon}{2}, \frac{5}{4}+\frac{\epsilon}{2}
\end{array} \mid-t^{2}\right)=\frac{\hat{K}}{2^{1+4 \epsilon} t^{1-\varepsilon}} I_{1}^{(2)}(t) \text {, } \\
& J_{1,1,2}^{(2)}(t)=-\frac{1}{2 \varepsilon^{2}(1-\varepsilon)}{ }_{4} F_{3}\left(\begin{array}{c}
1, \frac{1}{2}+\epsilon, \frac{\epsilon}{2}, \epsilon \\
\frac{3}{2}-\frac{\epsilon}{2}, \frac{1}{4}+\frac{\epsilon}{2}, \frac{3}{4}+\frac{\epsilon}{2}
\end{array} \mid-t^{2}\right)=-\frac{1}{2 \varepsilon^{2}(1-\varepsilon)} \\
& +\frac{1}{(1-2 \varepsilon)} \frac{\hat{K}}{2^{4 \epsilon} t^{1-\varepsilon}} \tilde{I}_{2}^{(2)}(t) \text {, } \\
& J_{1,1,1}^{(2)}(t)=-\frac{1}{\varepsilon^{2}(1-\varepsilon)(1-2 \varepsilon)}{ }_{4} F_{3}\left(\begin{array}{c}
1,-\frac{1}{2}+\epsilon, \frac{\epsilon}{2}, \epsilon \\
\frac{3}{2}-\frac{\epsilon}{2}, \frac{1}{4}+\frac{\epsilon}{2}, \frac{3}{4}+\frac{\epsilon}{2}
\end{array} \mid-t^{2}\right) \\
& =-\frac{1}{(1-\varepsilon)(1-2 \varepsilon) \varepsilon^{2}}-\frac{2^{2-4 \epsilon} \hat{K}}{(1-2 \varepsilon)^{2} t^{1-\varepsilon}} \tilde{I}_{3}^{(2)}(t), \\
& J_{1,2,2}^{(3)}(t)=\frac{1+\varepsilon}{\varepsilon(2-\varepsilon)(3+2 \varepsilon)}{ }_{4} F_{3}\left(\begin{array}{c}
1, \frac{3}{2}+\frac{\epsilon}{2}, 1+\epsilon, \frac{3}{2}+\epsilon \\
2-\frac{\epsilon}{2}, \frac{5}{4}+\frac{\epsilon}{2}, \frac{7}{4}+\frac{\epsilon}{2}
\end{array} \mid-t^{2}\right)=-\frac{\hat{K}}{2^{2+4 \epsilon} \varepsilon t^{2}} I_{1}^{(3)}(t), \\
& J_{1,1,2}^{(3)}(t)=-\frac{1}{\varepsilon(2-\varepsilon)(1+2 \varepsilon)}{ }_{4} F_{3}\left(\begin{array}{c}
1, \frac{1}{2}+\frac{\epsilon}{2}, 1+\epsilon, \frac{1}{2}+\epsilon \\
2-\frac{\epsilon}{2}, \frac{3}{4}+\frac{\epsilon}{2}, \frac{5}{4}+\frac{\epsilon}{2}
\end{array} \mid-t^{2}\right)=-\frac{\hat{K}}{2^{1+4 \epsilon}(1-2 \varepsilon) \varepsilon t^{2}} I_{2}^{(3)}(t), \\
& J_{1,1,1}^{(3)}(t)=-\frac{1}{\varepsilon^{2}(2-\varepsilon)(1+2 \varepsilon)}{ }_{4} F_{3}\left(\begin{array}{c}
1, \frac{1}{2}+\frac{\epsilon}{2}, \epsilon, \frac{1}{2}+\epsilon \\
2-\frac{\epsilon}{2}, \frac{3}{4}+\frac{\epsilon}{2}, \frac{5}{4}+\frac{\epsilon}{2}
\end{array} \mid-t^{2}\right)=\frac{1}{\varepsilon^{2}(2-\varepsilon)(1+2 \varepsilon)} \\
& +\frac{2^{1-4 \epsilon} \hat{K}}{(1-2 \varepsilon)^{2} \varepsilon t^{2}} \tilde{I}_{3}^{(3)}(t)
\end{aligned}
$$

where $\hat{K}$ is defined as,

$$
\hat{K}=\frac{\Gamma(1-\varepsilon)}{\Gamma(1-2 \varepsilon) \Gamma(1+\varepsilon)},
$$


while the factors $I_{j}^{(i)}(t)$ and $\tilde{I}_{3}^{(i)}(t)(j=1,2),(i=1,3)$ represent the relevant double integrals,

$$
I_{j}^{(i)}(t)=I_{j, 1}^{(i)}(t)-\frac{2 \epsilon}{1+i} I_{j, 2}^{(1)}(t), \tilde{I}_{3}^{(i)}(t)=I_{3,1}^{(i)}(t)-\frac{2 \epsilon}{1+i} I_{3,2}^{(i)}(t),
$$

with,

$$
\begin{aligned}
& I_{1,1}^{(1)}(t)=\int_{0}^{1} d p p^{\epsilon-1}(1-p)^{-\epsilon-\frac{1}{2}}\left(\left(p^{2} t^{2}+1\right)^{-\frac{1}{2}}-1\right), \\
& I_{1,2}^{(1)}(t)=\int_{0}^{1} d p p^{\epsilon-1}(1-p)^{-\epsilon-\frac{1}{2}}\left(p^{2} t^{2}+1\right)^{-\frac{1}{2}} J^{(1)}(q(p)), \\
& I_{2,1}^{(1)}(t)=\int_{0}^{1} d p p^{\epsilon-2}(1-p)^{-\epsilon-\frac{1}{2}}\left(\left(p^{2} t^{2}+1\right)^{\frac{1}{2}}-1\right), \\
& I_{2,2}^{(1)}(t)=\int_{0}^{1} d p p^{\epsilon-2}(1-p)^{-\epsilon-\frac{1}{2}}\left(p^{2} t^{2}+1\right)^{\frac{1}{2}} J^{(1)}(q(p)), \\
& \tilde{I}_{3,1}^{(1)}(t)=\int_{0}^{1} d p p^{\epsilon-3}(1-p)^{-\epsilon+\frac{1}{2}}\left(\left(p^{2} t^{2}+1\right)^{\frac{1}{2}}-1-\frac{(p t)^{2}}{2}\right), \\
& \tilde{I}_{3,2}^{(1)}(t)=\int_{0}^{1} d p p^{\epsilon-3}(1-p)^{-\epsilon+\frac{1}{2}}\left(p^{2} t^{2}+1\right)^{\frac{1}{2}} \tilde{J}^{(1)}(q(p)), \\
& I_{1}^{(2)}(t)=\int_{0}^{1} d p p^{3 \epsilon-1}(1-p)^{-\epsilon-\frac{1}{2}}\left(p^{2} t^{2}+1\right)^{-\epsilon-\frac{1}{2}} J^{(2)}(p t), \\
& I_{2}^{(2)}(t)=\int_{0}^{1} d p p^{3 \epsilon-2}(1-p)^{-\epsilon-\frac{1}{2}}\left(p^{2} t^{2}+1\right)^{-\epsilon+\frac{1}{2}} J^{(2)}(p t), \\
& \tilde{I}_{2}^{(2)}(t)=\int_{0}^{1} d p p^{3 \epsilon-2}(1-p)^{-\epsilon-\frac{1}{2}}\left(p^{2} t^{2}+1\right)^{-\epsilon+\frac{1}{2}} \tilde{J}^{(2)}(p t), \\
& \tilde{I}_{3}^{(2)}(t)=\int_{0}^{1} d p p^{3 \epsilon-3}(1-p)^{-\epsilon+\frac{1}{2}}\left(p^{2} t^{2}+1\right)^{-\epsilon+\frac{1}{2}} \tilde{J}^{(2)}(p t), \\
& I_{1,1}^{(3)}(t)=\int_{0}^{1} d p p^{2 \epsilon-1}(1-p)^{-\epsilon-\frac{1}{2}}\left(\left(p^{2} t^{2}+1\right)^{-\frac{\epsilon}{2}-\frac{1}{2}}-1\right), \\
& I_{1,2}^{(3)}(t)=\int_{0}^{1} d p p^{2 \epsilon-1}(1-p)^{-\epsilon-\frac{1}{2}}\left(p^{2} t^{2}+1\right)^{-\frac{\epsilon}{2}-\frac{1}{2}} J^{(3)}(q(p)), \\
& I_{2,1}^{(3)}(t)=\int_{0}^{1} d p p^{2 \epsilon-2}(1-p)^{-\epsilon-\frac{1}{2}}\left(\left(p^{2} t^{2}+1\right)^{\frac{1}{2}-\frac{\epsilon}{2}}-1\right), \\
& I_{2,2}^{(3)}(t)=\int_{0}^{1} d p p^{2 \epsilon-2}(1-p)^{-\epsilon-\frac{1}{2}}\left(p^{2} t^{2}+1\right)^{\frac{1}{2}-\frac{\epsilon}{2}} J^{(3)}(q(p)), \\
& \tilde{I}_{3,1}^{(3)}(t)=\int_{0}^{1} d p p^{2 \epsilon-3}(1-p)^{-\epsilon+\frac{1}{2}}\left(\left(p^{2} t^{2}+1\right)^{\frac{1}{2}-\frac{\epsilon}{2}}-1-(1-\varepsilon) \frac{(p t)^{2}}{2}\right), \\
& \tilde{I}_{3,2}^{(3)}(t)=\int_{0}^{1} d p p^{2 \epsilon-3}(1-p)^{-\epsilon+\frac{1}{2}}\left(p^{2} t^{2}+1\right)^{\frac{1}{2}-\frac{\epsilon}{2}} \tilde{J}^{(3)}(q(p)),
\end{aligned}
$$

where

$$
\begin{array}{rlrl}
J^{(1)}(q) & =q^{\epsilon} \int_{0}^{q} d z\left((1-z)^{-\frac{1}{2}}-1\right) z^{-\epsilon-1}, & J^{(2)}(p t)=\int_{0}^{p t} d z z^{-\epsilon}\left(z^{2}+1\right)^{\epsilon-\frac{1}{2}} \\
J^{(3)}(q)=q^{\frac{\epsilon}{2}} \int_{0}^{q} d z\left((1-z)^{-\frac{\epsilon}{2}-\frac{1}{2}}-1\right) z^{-\frac{\epsilon}{2}-1}, & \tilde{J}^{(1)}(q)=J^{(1)}(q)-\frac{(p t)^{2}}{2(1-\varepsilon)} \\
\tilde{J}^{(2)}(p t)=J^{(2)}(p t)-\frac{1}{\left(p^{2} t^{2}+1\right)^{\frac{1}{2}-\epsilon}} \frac{(p t)^{1-\varepsilon}}{1-\varepsilon}, & \tilde{J}^{(3)}(q)=J^{(3)}(q)-\frac{(1+\varepsilon)}{(2-\varepsilon)}(p t)^{2}
\end{array}
$$


and

$$
q(p)=\frac{p^{2} t^{2}}{p^{2} t^{2}+1} .
$$

Integrals with tildes are used when the corresponding integrals have singularities for small $p$ values in (3.6) and for small $z$ values in (3.7). They are constructed from the corresponding integrals by extracting the leading asymptotics of subintegral expressions for small $p$ and for small $z$, respectively, and, therefore, they are finite.

In appendix D we present results for the integrals discussed in this paper in terms of one-fold integral over polylogarithmic expressions, up to $\mathcal{O}(\varepsilon)$. In principle, results of this form can be extended to any order of $\varepsilon$ (see for example [110] and [64, 67]).

\section{All orders result in terms of elliptic polylogarithms}

In this section we derive eMPL representations for the sunrise integrals $J_{1,2,2}, J_{1,1,2}$ and $J_{1,1,1}$ valid to all orders of the dimensional regulator. Specifically, we start with a short review of eMPLs, discussing their definition and the basic analytic properties. We then discuss the general structure of the integral representations presented in the previous section and we show that, by defining a new integration variable, their dependence on the relevant elliptic curve can be made explicit. We conclude by discussing the general solution strategy used to express these integrals in terms of eMPLs to all orders of the dimensional regulator, and present our final results.

\subsection{Elliptic polylogarithms}

We are interested in the computation of iterated integrals of the form,

$$
\int_{0}^{x} d x_{1} R_{1}\left(x_{1}, y\left(x_{1}\right)\right) \int_{0}^{x_{1}} d x_{2} R_{2}\left(x_{2}, y\left(x_{2}\right)\right) \cdots \int_{0}^{x_{n-1}} d x_{n} R_{n}\left(x_{n}, y\left(x_{n}\right)\right),
$$

where $R_{i}$ are rational functions of their arguments and $y(x)$ is an elliptic curve,

$$
y(x)=\sqrt{\left(x-a_{1}\right)\left(x-a_{2}\right)\left(x-a_{3}\right)\left(x-a_{4}\right)} .
$$

All iterated integrals of the form (4.1) can be expressed in terms of eMPLs. In the complex plane, eMPLs are defined as

$$
E_{4}\left(\begin{array}{l}
n_{1}, \ldots, n_{k} \\
c_{1}, \ldots, c_{k}
\end{array} ; x\right)=\int_{0}^{x} d t \varphi_{n_{1}}\left(c_{1}, t\right) E_{4}\left(\begin{array}{c}
n_{2}, \ldots, n_{k} \\
c_{2}, \ldots, c_{k}
\end{array} ; t\right)
$$

with $n_{i} \in \mathbb{Z}$ and $c_{i} \in \mathbb{C} \cup\{\infty\}$. The recursion starts at $E_{4}(; x)=1$. By construction, the kernels $\varphi_{n}(c, x)$ have at most simple poles, and they are (see [35] for a detailed discussion), ${ }^{1}$

$$
\begin{aligned}
\varphi_{0}(0, x) & =\frac{c_{4}}{y(x)}, & & \\
\varphi_{1}(c, x) & =\frac{1}{x-c}, & \varphi_{-1}(c, x) & =\frac{y(c)}{(x-c) y(x)}-\left(\delta_{c 0}+\delta_{c 1}\right) \frac{1}{x-c}, \\
\varphi_{-1}(\infty, x) & =\frac{x}{y(x)}, & \varphi_{1}(\infty, x) & =\frac{c_{4}}{y(x)} Z_{4}(x),
\end{aligned}
$$

\footnotetext{
${ }^{1}$ In what follows we will encounter eMPLs evaluated at $x=0$ and $x=1$ and in order to make possible divergent behaviour at these points manifest we regulated kernel $\varphi_{-1}(c, x)$ in $x=0$ and $x=1$ when $c=0$ and $c=1$ respectively (see section 4.2 ).
} 


$$
\begin{aligned}
\varphi_{n}(\infty, x) & =\frac{c_{4}}{y(x)} Z_{4}^{(n)}(x), \quad \varphi_{-n}(\infty, x)=\frac{x}{y(x)} Z_{4}^{(n-1)}(x)-\frac{\delta_{n 2}}{c_{4}}, \\
\varphi_{n}(c, x) & =\frac{1}{x-c} Z_{4}^{(n-1)}(x)-\delta_{n 2} \Phi_{4}(x), \\
\varphi_{-n}(c, x) & =\frac{y(c)}{(x-c) y(x)} Z_{4}^{(n-1)}(x), \quad(n>1)
\end{aligned}
$$

where $y(c)$ and $c_{4}$ are independent of $x$ with,

$$
c_{4}=\frac{1}{2} \sqrt{a_{13} a_{24}} \quad \text { with } \quad a_{i j}=a_{i}-a_{j} .
$$

Moreover we define,

$$
E_{4}(\underset{\overrightarrow{0}}{\overrightarrow{1}} ; x) \equiv \frac{\log (x)^{n}}{n !},
$$

where $\overrightarrow{1}$ and $\overrightarrow{0}$ are vectors with entries equal to 1 and 0 respectively, and $n=\operatorname{length}(\overrightarrow{1})=$ length $(\overrightarrow{0})$. The function $Z_{4}(x)$ is defined by first introducing an auxiliary function $\Phi_{4}(x)$,

$$
\Phi_{4}(x) \equiv \widetilde{\Phi}_{4}(x)+4 c_{4} \frac{\eta_{1}}{\omega_{1}} \frac{1}{y}=\frac{1}{c_{4} y}\left(x^{2}-\frac{s_{1}}{2} x+\frac{s_{2}}{6}\right)+4 c_{4} \frac{\eta_{1}}{\omega_{1}} \frac{1}{y},
$$

where $\eta_{1}, \omega_{1}, s_{1}, s_{2}, c_{4}$ are independent of $x$ and they are defined in [35], and,

$$
\widetilde{\Phi}_{4}(x)=\frac{1}{c_{4} y}\left(x^{2}-\frac{s_{1} x}{2}+\frac{s_{2}}{6}\right)
$$

whose primitive is,

$$
Z_{4}(x)=\int_{a_{1}}^{x} d t \Phi_{4}(t)
$$

In the next sections we will see that, in our integral representations, the function $\Phi_{4}(x)$ appears only in the last (outer) integration, and only the case $Z_{4}^{(1)}(x)=Z_{4}(x)$ need to be considered.

Elliptic polylogarithms are a generalisation of ordinary multiple polylogarithms (MPLs), defined recursively as,

$$
G\left(a_{1}, a_{2}, \ldots, a_{n} ; x\right)=\int_{0}^{x} \frac{d t}{t-a_{1}} G\left(a_{2}, \ldots, a_{n}, t\right)
$$

with $G(; x) \equiv 1$ and,

$$
G(\overrightarrow{0}, x) \equiv \frac{\log (x)^{n}}{n !} .
$$

By definition we see that MPLs are a subset of eMPLs,

$$
E_{4}\left(\begin{array}{c}
1, \ldots, 1 \\
c_{1}, \ldots, c_{n}
\end{array} ; x\right)=G\left(c_{1}, c_{2}, \ldots, c_{n} ; x\right),
$$

where $c_{i} \neq \infty$.

The appearance of the function $Z_{4}(x)$ and its regularised powers $Z_{4}(x)^{(n)}$ is due to the requirement that, in analogy to MPLs, integration kernels have at most simple poles [35]. The integrals considered in this paper do not involve $Z_{4}(x)$ and its regularised powers. 
As for all iterated integrals, eMPLs satisfy a shuffle algebra, with the shuffle product defined as,

$$
E_{4}\left(\begin{array}{ccc}
a_{1} & \ldots & a_{n} \\
a_{1}^{\prime} & \ldots & a_{n}^{\prime}
\end{array} ; x\right) E_{4}\left(\begin{array}{ccc}
b_{1} & \ldots & b_{m} \\
b_{1}^{\prime} & \ldots & b_{m}^{\prime}
\end{array} ; x\right)=\sum_{\vec{c}=\vec{a} \uplus \vec{b}} E_{4}\left(\begin{array}{ccc}
c_{1} & \ldots & c_{n+m} \\
c_{1}^{\prime} & \ldots & c_{n+m}^{\prime}
\end{array} ; x\right) .
$$

The vector $\vec{c}$ is the vector obtained by performing all the shuffles of $\vec{a}$ and $\vec{b}$, preserving the ordering of the elements of $\vec{a}$ and $\vec{b}$ respectively.

\subsection{Regularisation}

As we will see in the next sections we are interested in computing definite integrals of the form,

$$
\int_{0}^{1} f(x) d x=F(1)-F(0), \quad \frac{\partial F(x)}{\partial x}=f(x) .
$$

In some cases individual functions inside the primitive develop divergences when evaluated at the integration bounds, and in order to compute the definite integral one needs to perform two limits,

$$
\int_{0}^{1} f(x) d x=\lim _{x \rightarrow 1} F(x)-\lim _{x \rightarrow 0} F(x) .
$$

We illustrate how the limits are performed for the case,

$$
F(x)=E_{4}\left(\begin{array}{l}
-1 \\
-1
\end{array} ; 1\right) E_{4}\left(\begin{array}{l}
1 \\
1
\end{array} ; x\right)+E_{4}\left(\begin{array}{c}
1-1 \\
0-1
\end{array}-x\right)-E_{4}\left(\begin{array}{c}
1-1 \\
1-1
\end{array} ; x\right) .
$$

From eq. (4.16) we see that $F(0)=0$, while two eMPLs, $E_{4}\left(\begin{array}{l}1 \\ 1\end{array} ; x\right)$ and $E_{4}\left(\begin{array}{l}1-1 \\ 1-1\end{array}-x\right)$, are divergent for $x=1$, therefore the computation of the integral (4.14) requires the computation of the following limit,

$$
\int_{0}^{1} f(x) d x=\lim _{x \rightarrow 1} F(x) .
$$

The limits can be computed by performing shuffle regularisation. Specifically, we first use shuffle identities to isolate the logarithmic divergences,

$$
E_{4}\left(\begin{array}{ll}
1 & -1 \\
1 & -1
\end{array} ; x\right)=E_{4}\left(\begin{array}{l}
-1 \\
-1
\end{array} ; x\right) E_{4}\left(\begin{array}{l}
1 \\
1
\end{array} ; x\right)-E_{4}\left(\begin{array}{cc}
-1 & 1 \\
-1 & 1
\end{array} ; x\right),
$$

and by inserting this result in (4.16) we find,

$$
F(x)=E_{4}\left({ }_{-1}^{-1} ; 1\right) E_{4}\left(\begin{array}{l}
1 \\
1
\end{array} ; x\right)-E_{4}\left({ }_{-1}^{-1} ; x\right) E_{4}\left(\begin{array}{l}
1 \\
1
\end{array} ; x\right)+E_{4}\left(\begin{array}{ll}
-1 & 1 \\
-1 & 1
\end{array} ; x\right)+E_{4}\left(\begin{array}{ll}
1 & -1 \\
0 & -1
\end{array} ; x\right) .
$$

The divergences in $x=1$ are now only carried by factors of $E_{4}\left(\frac{1}{1} ; x\right)=\log (1-x)$. However it is easy to see that the divergences explicitly cancel in the limit, and we obtain,

$$
\int_{0}^{1} f(x) d x=\lim _{x \rightarrow 1} F(x)=E_{4}\left(\begin{array}{cc}
-1 & 1 \\
-1 & 1
\end{array} 1\right)+E_{4}\left(\begin{array}{cc}
1 & -1 \\
0 & -1
\end{array} ; 1\right) .
$$

We remark that for the choice of the integration kernels (4.4), divergent eMPLs at the integration bounds considered in this work, i.e. 0 and 1 , are those of the form,

$$
E_{4}\left(\begin{array}{c}
1 \\
1 \\
1
\end{array} ; 1\right), \quad E_{4}\left(\begin{array}{ccc}
1 & \ldots & 1 \\
0 & \ldots & 0
\end{array} ; 0\right), \quad\left(\begin{array}{l}
\vec{m} \\
\vec{n}
\end{array}\right) \neq\left(\begin{array}{c}
1, \ldots, 1 \\
0, \ldots 0
\end{array}\right),
$$




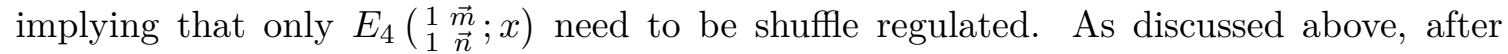
shuffle regularisation, the diverging terms are powers of $E_{4}\left(\begin{array}{l}1 \\ 1\end{array} ; x\right)$ and $E_{4}\left(\begin{array}{l}1 \\ 0\end{array} ; x\right)$, and a manifestly finite result is obtained by taking the limit $x \rightarrow 1$ and $x \rightarrow 0$ as described above. For a finite result all divergent factors are multiplied by a vanishing expression in the limits.

In what follows we use a more direct and equivalent procedure to compute definite integrals involving singular primitives. Specifically, we formally compute definite integrals as the difference of the primitive evaluated at the integration bounds. Finally, we shuffle regulate the result and set to zero all divergent logarithms.

\subsection{Elliptic polylogarithms and all orders result}

In this section we show that the double integrals of section 3 can be expressed as a $\mathbb{Q}$-linear combinations of eMPLs to all orders of the dimensional regulator. We start by observing that the integrals of eq. (3.6) have the following general form, ${ }^{2}$

$$
\int_{0}^{1} d p \sum_{i=1}^{n_{1}} f_{1, i}(p) g_{1, i}(p)^{\epsilon} \int_{0}^{h(p)} \sum_{j=1}^{n_{2}} f_{2, j}(z) g_{2, j}(z)^{\epsilon} d z, \quad n_{1}, n_{2} \in \mathbb{N},
$$

where $f_{1, i}(p), g_{1, j}(p)$ are algebraic functions of $p$, with algebraic factors,

$$
\sqrt{1-p}, \sqrt{1+t^{2} p^{2}}, \quad 0<p<1,0<t<1,
$$

$f_{2, j}(z), g_{2, j}(z)$ are algebraic functions with algebraic factors,

$$
\sqrt{1-z}, \sqrt{1+z^{2}}, \quad 0<z<1,
$$

while $h(p)$ is a rational function of $p$. The dependence on the relevant elliptic curve can be made explicit for all integrands by the variable change,

$$
p(x)=1-x^{2}, \quad 0<x<1 .
$$

After the variable change we can write,

$$
-\int_{0}^{1} d x \sum_{i=1}^{n_{1}} f_{1, i}^{\prime}(x, y(x)) g_{1, i}^{\prime}(x, y(x))^{\epsilon} \int_{0}^{h(p(x))} \sum_{j=1}^{n_{2}} f_{2, j}(z) g_{2, j}(z)^{\epsilon} d z,
$$

where the elliptic curve is,

$$
y(x)=\sqrt{\frac{1}{t^{2}}+\left(1-x^{2}\right)^{2}},
$$

and,

$$
f_{1, i}^{\prime}(x, y(x))=\frac{d p(x)}{d x} f_{1, i}(p(x)), \quad g_{1, i}^{\prime}(x, y(x))=g_{1, i}(p(x)) .
$$

For integral $J_{1,2,2}, f_{1, i}^{\prime}(x, y(x))$ is a rational function on the elliptic curve $y(x)$ with at most simple poles, implying that it can be expressed as a $\mathbb{Q}$-linear combination of kernels $\varphi_{0}(0, x)$,

\footnotetext{
${ }^{2}$ Integrals $I_{i, 1}^{(1)}$ and $I_{i, 1}^{(3)}(i=1,2,3)$ are in fact one-fold integrals, but for the general discussion of this section includes trivially the simpler case.
} 
$\varphi_{1}(c, x), \varphi_{-1}(c, x), \varphi_{-1}(\infty, x)$. For integrals $J_{1,1,2}$ and $J_{1,1,1}$ the function $f_{1, i}^{\prime}(x, y(x))$ depends also on $\Phi_{4}(x)$, as shown in appendix $\mathrm{C}$, and the solution of the integrals in terms of eMPLs introduces the additional kernels $\varphi_{1}(\infty, x), \varphi_{-2}(\infty, x), \varphi_{ \pm 2}(c, x)$, and it requires the application of integration by parts identities (see appendix E). In order to introduce the formalism used in this paper we continue the discussion of this section considering quantities related to integral $J_{1,2,2}$. The derivation of the results for all the integrals is presented in the next sections and the appendices. The function $g_{1, i}^{\prime}(x, y(x))$ takes the form,

$$
g_{1, i}^{\prime}(x, y(x))=y(x)^{\alpha} R_{1, i}(x), \quad \alpha \in \mathbb{N},
$$

where $R_{1, i}$ is a rational function, which implies that upon expanding in powers of $\epsilon$, the resulting logarithmic factor can be evaluated in terms of eMPLs of the form $E_{4}\left({ }_{c}^{1} ; x\right)$, i.e. ordinary MPLs. The $\epsilon$ expansion is performed according to,

$$
g_{1, i}^{\prime}(x, y(x))^{\epsilon}=\sum_{i=0}^{\infty} \frac{\log \left(g_{1, i}^{\prime}(x, y(x))\right)^{i} \epsilon^{i}}{i !} .
$$

The logarithm can be expressed as $\mathbb{Q}$-linear combinations of eMPLs, by using the general identity,

$$
\log (a(x, y(x)))=\log (a(0, y(0)))+\int_{0}^{x} d z \frac{d a(z, y(z))}{d z} \frac{1}{a(z, y(z))},
$$

for regular and non-vanishing $a(0, y(0))$. As anticipated, eq. (4.29) implies that the integrand on the right hand side can be expressed as a $\mathbb{Q}$-linear combination of $\varphi_{1}(c, x)$. For a subset of integrals we have that $a(x, y(x))=x^{\beta} r(x, y(x))$, with regular and non-vanishing $r(0, y(0)), \beta \neq 0$, and the identity above is ill-defined. Nonetheless in these cases we simply have,

$$
\log (a(x, y(x)))=\log (r(0, y(0)))+\beta \log (x)+\int_{0}^{x} d z \frac{d r(z, y(z))}{d z} \frac{1}{r(z, y(z))} .
$$

Results of the same form can be obtained for the inner integral. This is achieved by changing the upper integration bound to $x$, by using the fact that,

$$
\int_{0}^{a(x)} b(z) d z=\int_{0}^{a(1)} b(z)+\int_{1}^{x} d z \frac{\partial}{\partial x} \int_{0}^{a(x)} b(z) d z
$$

and

$$
\frac{\partial}{\partial x} \int_{0}^{a(x)} b(z) d z=b(a(z)) \frac{d b(z)}{d z}
$$

where the choice of unit lower integration bound is conventional at this point, and will be clarified later. For the case at hand, the identities above imply,

$$
\begin{aligned}
\int_{0}^{h(p(x))} \sum_{j=1}^{n_{2}} f_{2, j}(z) g_{2, j}(z)^{\epsilon} d z= & \int_{1}^{x} \sum_{j, k=1}^{n_{2}} f_{2, j}(h(p(z))) g_{2, j}(h(p(z)))^{\epsilon} \frac{d\left(f_{2, k}(z) g_{2, k}(z)^{\epsilon}\right)}{d z} d z \\
& +\int_{0}^{h(p(1))} \sum_{j=1}^{n_{2}} f_{2, j}(z) g_{2, j}(z)^{\epsilon} d z
\end{aligned}
$$


For the $h(p(x))$ considered in this work, $h(p(1))=0$, so that the last integral on the right hand side vanishes. Moreover, for the relevant $f_{2, j}(z)$ and $g_{2, j}(z)$, we can write,

$$
\int_{1}^{x} \sum_{j, k=1}^{n_{2}} f_{2, j}(h(p(z))) g_{2, j}(h(p(z)))^{\epsilon} \frac{d\left(f_{2, k}(z) g_{2, k}(z)^{\epsilon}\right)}{d z} d z=\int_{1}^{x} \sum_{i=1}^{n} f_{2, i}^{\prime}(z, y(z)) g_{2, i}^{\prime}(z, y(z))^{\epsilon} d z
$$

where $f_{2, i}^{\prime}(z, y(z))$ are rational functions on $y(z)$ with at most simple poles while $g_{2, i}^{\prime}(z, y(z))$ have the same form as eq. (4.29). Logarithms and prefactors may be expressed as $\mathbb{Q}$ linear combinations of eMPLs and integration kernels respectively as discussed in the previous paragraph.

This analysis implies that integrals of the form eq. (4.22) formally evaluate to,

$$
\sum_{l=1}^{n^{\prime}} C_{l} \sum_{i, j=0}^{\infty} \frac{\epsilon^{i+j}}{i ! j !} \int_{0}^{1} d x k_{1, l}(x) L_{1, l}(x)^{i} \int_{1}^{x} d z k_{2, l}(z) L_{2, l}(z)^{j}, \quad n^{\prime} \in \mathbb{N}, C_{l} \in \mathbb{Q},
$$

where $L_{i, j}(x)$ are $\mathbb{Q}$-linear combinations of eMPLs of depth one, while $k_{i, j}(x)$ are $\mathbb{Q}$-linear combinations of integration kernels. These integrals can be directly evaluated in terms of eMPLs by shuffle expanding products of eMPLs of the integrands, and by using recursively the definition of eMPLs.

Eq. (4.37) is not well defined in general, since double integrals might be individually divergent, while only the full linear combination corresponds to a finite result. Nonetheless a manifestly finite result can be obtained by performing shuffle regularisation as described in 4.2. More precisely, diverging powers of logarithms (in our case powers of $E_{4}\left(\begin{array}{l}1 \\ 0\end{array} ; 0\right)$ and $\left.E_{4}\left(\begin{array}{l}1 \\ 1\end{array} ; 1\right)\right)$ are isolated by using shuffle identities and are subsequently set to zero.

In order to make the notation more compact, and make properties of the result in terms of eMPLs manifest, we use the following notation for the double integrals of (4.37). By denoting a primitive of $k_{i, j}(x)$ as $K_{i, j}(x)$, and by defining the bilinear $*$-operator as,

$$
E_{4}\left(\begin{array}{l}
\vec{n} \\
\vec{c}
\end{array} ; x\right) * E_{4}\left(\begin{array}{l}
\vec{m} \\
\vec{d}
\end{array} ; x\right)=E_{4}\left(\begin{array}{l}
\vec{n} \\
\vec{c}
\end{array} \vec{d} ; x\right),
$$

we can write

$$
\underset{0,1}{\operatorname{Reg}} \sum_{l=1}^{n^{\prime}} C_{l} \sum_{i, j=0}^{\infty} \frac{\epsilon^{i+j}}{i ! j !} K_{1, l}(x) * L_{1, l}(x)^{i}\left[K_{2, l}(z) * L_{2, l}(z)^{j}\right]_{1}^{x},
$$

where all products of eMPLs are shuffle expanded before applying the $*$-operator, and these operations are carried out for the inner square brackets first. Finally, the lower and upper scripts applied to the square brackets denote the following operation,

$$
[F(x)]_{1}^{x}=F(x)-F(1),
$$

while the regularisation operator is,

$$
\operatorname{Reg}_{0,1} F(x)=\operatorname{Reg}_{x \rightarrow 1} F(x)-\operatorname{Reg}_{x \rightarrow 0} F(x),
$$

where $\operatorname{Reg}_{x \rightarrow x_{0}}, x_{0} \in\{0,1\}$ denotes the regularised limit operation described in section 4.2. 


\subsection{Sunsets}

By applying the procedure described above we obtain one of the main results of this paper, i.e. an explicit expression for the integrals of eq. (3.6) in terms of eMPLs valid to all orders of the dimensional regulator.

\subsection{1 $J_{1,2,2}$}

Combining eqs. (3.3), (E.1) and (E.2) to the right hand side of eq. (3.2), we obtain the following expression for $J_{1,2,2}$,

$$
\begin{aligned}
M^{2} J_{1,2,2} & =\hat{N}_{1} \frac{\hat{K}_{1}}{4 t^{2}} \hat{J}_{1,2,2}, \\
\hat{J}_{1,2,2} & =\left[\frac{1}{\varepsilon} I_{1}^{(1)}(t)+\left(\frac{t^{2}}{2}\right)^{\varepsilon} I_{1}^{(2)}(t)-\frac{1}{\varepsilon}\left(\frac{t}{2}\right)^{\varepsilon} I_{1}^{(3)}(t)\right] \\
& =\left[\frac{1}{\varepsilon} I_{1,1}^{(1)}(t)-I_{1,2}^{(1)}(t)+\left(\frac{t^{2}}{2}\right)^{\varepsilon} I_{1}^{(2)}(t)-\left(\frac{t}{2}\right)^{\varepsilon}\left(\frac{1}{\varepsilon} I_{1,1}^{(3)}(t)-\frac{1}{2} I_{1,2}^{(3)}(t)\right)\right],
\end{aligned}
$$

where,

$$
\begin{aligned}
& I_{1,1}^{(1)}(t)=\underset{0,1}{\operatorname{Reg}} \sum_{i=0}^{\infty} \frac{\epsilon^{i}}{i !} K_{1} * L_{1}^{i}, \quad, \\
& I_{1,2}^{(1)}(t)=\underset{0,1}{\operatorname{Reg}} \sum_{i, j=0}^{\infty} \frac{\epsilon^{i+j}}{i ! j !} K_{2} * L_{3}^{i}\left[K_{3} * L_{2}^{j}\right]_{1}^{x} \text {, } \\
& I_{1}^{(2)}(t)=\underset{0,1}{\operatorname{Reg}} \sum_{i, j=0}^{\infty} \frac{\epsilon^{i+j}}{i ! j !} K_{4} * L_{5}^{i}\left[K_{5} * L_{4}^{j}\right]_{1}^{x} \text {, } \\
& I_{1,1}^{(3)}(t)=\underset{0,1}{\operatorname{Reg}} \sum_{i=0}^{\infty} \frac{\epsilon^{i}}{i !} K_{6} * L_{6}^{i}+\underset{0,1}{\operatorname{Reg}} \sum_{i=0}^{\infty} \frac{\epsilon^{i}}{i !} K_{4} * L_{7}^{i}, \\
& I_{1,2}^{(3)}(t)=\underset{0,1}{\operatorname{Reg}} \sum_{i, j=0}^{\infty} \frac{\epsilon^{i+j}}{i ! j !} K_{7} * L_{8}^{i}\left[K_{9} * L_{4}^{j}\right]_{1}^{x}+\underset{0,1}{\operatorname{Reg}} \sum_{i, j=0}^{\infty} \frac{\epsilon^{i+j}}{i ! j !} K_{7} * L_{8}^{i}\left[K_{8} * L_{9}^{j}\right]_{1}^{x} \text {, }
\end{aligned}
$$

where $K_{i}$ and $L_{i}$ are depth one eMPLs and their definition is provided in appendix C.

Since the integrals $I_{1}^{(2)}(t)$ and $I_{1}^{(3)}(t)$ contribute to $\hat{J}_{1,2,2}$ with the corresponding factors, it is convenient to present also

$$
\begin{aligned}
& \left(\frac{t^{2}}{2}\right)^{\varepsilon} I_{1}^{(2)}(t)=\operatorname{Reg}_{0,1} \sum_{i, j=0}^{\infty} \frac{\epsilon^{i+j}}{i ! j !} K_{4} * \hat{L}_{5}^{i}\left[K_{5} * L_{4}^{j}\right]_{1}^{x}, \\
& \left(\frac{t}{2}\right)^{\varepsilon} I_{1,1}^{(3)}(t)=\operatorname{Reg}_{0,1}^{\infty} \sum_{i=0}^{\infty} \frac{\epsilon^{i}}{i !} K_{6} * \hat{L}_{6}^{i}+\underset{0,1}{\operatorname{Reg}} \sum_{i=0}^{\infty} \frac{\epsilon^{i}}{i !} K_{4} * \hat{L}_{7}^{i}, \\
& \left(\frac{t}{2}\right)^{\varepsilon} I_{1,2}^{(3)}(t)=\underset{0,1}{\operatorname{Reg}} \sum_{i, j=0}^{\infty} \frac{\epsilon^{i+j}}{i ! j !} K_{7} * \hat{L}_{8}^{i}\left[K_{9} * L_{4}^{j}\right]_{1}^{x}+\underset{0,1}{\operatorname{Reg}} \sum_{i, j=0}^{\infty} \frac{\epsilon^{i+j}}{i ! j !} K_{7} * \hat{L}_{8}^{i}\left[K_{8} * L_{9}^{j}\right]_{1}^{x},
\end{aligned}
$$

where,

$$
\hat{L}_{5}=l_{5}+2 \log t-\log 2, \quad \hat{L}_{k}=l_{k}+\log t-\log 2 \quad(k=6,7,8) .
$$


We see that the integral $J_{1,2,2}$ in the form of the elliptic integrals is finite since $K_{1}=$ $K_{4}+K_{6}$ (see appendix E) and,

$$
I_{1,1}^{(1)}(t)-\left(\frac{t}{2}\right)^{\varepsilon} I_{1,1}^{(3)}(t)=\operatorname{Reg}_{0,1} \sum_{i=1}^{\infty} \frac{\epsilon^{i}}{i !} K_{1} *\left(L_{1}^{i}-\hat{L}_{6}^{i}\right)+\underset{0,1}{\operatorname{Reg}} \sum_{i=1}^{\infty} \frac{\epsilon^{i}}{i !} K_{2} *\left(L_{1}^{i}-\hat{L}_{7}^{i}\right) \sim O(\varepsilon) .
$$

\subsection{2 $J_{1,1,1}$ and $J_{1,1,2}$}

Combining eqs. (3.2) and (3.3), we obtain the following expression for $J_{1,1,2}$ and $J_{1,1,1}$,

$$
\begin{aligned}
& J_{1,1, j}=J_{1,1, j}^{\text {sing }}+J_{1,1, j}^{r e g}(j=1,2), \\
& J_{1,1,2}^{\text {sing }}=-\frac{\hat{N}_{2}}{2(1-\varepsilon) \varepsilon^{2}}, \quad J_{1,1,2}^{r e g}=\frac{\hat{K}_{1} \hat{N}_{1}}{2(1-2 \varepsilon) t^{2}} \hat{J}_{1,1,2} \\
& \hat{J}_{1,1,2}^{\mathrm{reg}}=\left[\frac{1}{\varepsilon} I_{2}^{(1)}(t)+\left(\frac{t^{2}}{2}\right)^{\varepsilon} \tilde{I}_{2}^{(2)}(t)-\frac{1}{\varepsilon}\left(\frac{t}{2}\right)^{\varepsilon} I_{2}^{(3)}(t)\right] \text {, } \\
& J_{1,1,1}^{\text {sing }}=\left\{\frac{m^{2}}{\varepsilon^{2}}\left[\frac{1}{(2-\varepsilon)(1+2 \varepsilon)}-\frac{(2 t)^{-\varepsilon}}{(1-\varepsilon)}\right]-\frac{M^{2}}{(1-\varepsilon)(1-2 \varepsilon) \varepsilon^{2}}\right\} \hat{N}_{2}, \\
& J_{1,1,1}^{\mathrm{reg}}=-M^{2} \frac{2 \hat{K}_{1} \hat{N}_{1}}{(1-2 \varepsilon)^{2} t^{2}} \hat{J}_{1,1,1}, \quad \hat{J}_{1,1,1}=\left[\frac{1}{\varepsilon} \tilde{I}_{3}^{(1)}(t)+\left(\frac{t^{2}}{2}\right)^{\varepsilon} \tilde{I}_{3}^{(2)}(t)-\frac{1}{\varepsilon}\left(\frac{t}{2}\right)^{\varepsilon} \tilde{I}_{3}^{(3)}(t)\right] \text {, }
\end{aligned}
$$

where

$$
\hat{N}_{2}=(2 t)^{\varepsilon-1} \hat{N}_{1}=\frac{\Gamma^{2}(1+\varepsilon)\left(\mu^{2}\right)^{2 \varepsilon}}{\left(M^{2}\right)^{2 \varepsilon}} .
$$

Results for $I_{2}^{(i)}(t), \tilde{I}_{2}^{(2)}(t), \tilde{I}_{3}^{(2)}(t)$ and $\tilde{I}_{3}^{(i)}(t)(i=1,3)$ shown in (3.5) and (3.6) can be expressed in terms of eMPLs by using the strategy of the previous sections and by applying integration by parts identities (see [35]). However, upon variable change (4.25), one encounters kernels of the form $\frac{x^{a}}{y(x)}$ with $a>2$, which render the integration by parts identities quite cumbersome. We show in appendix $\mathrm{C}$ that one can reduce these integrals to the computation of integrals $I_{21}^{(j)}(t)$ and $I_{31}^{(j)}(t)(j=1,2,3)$ (defined below in eqs. (4.54)(4.57) and (4.60) and (4.61)), which require considering only kernels with at most simple poles, and $\frac{x^{2}}{y(x)}$ (see appendix C), which has a double pole at infinity. The results presented in appendix $\mathrm{C}$ take the form,

$$
\begin{aligned}
& I_{2}^{(i)}(t)=\frac{1}{2(1-3 \varepsilon)}\left[(1-4 \varepsilon) I_{1}^{(i)}(t)-t^{2} I_{21}^{(i)}(t)+2(1-2 \varepsilon) t^{2} I_{31}^{(i)}(t)\right] \\
& \tilde{I}_{3}^{(i)}(t)=\frac{(1-2 \varepsilon)}{2(2-3 \varepsilon)}\left[2 t^{2}\left(I_{1}^{(i)}(t)-I_{31}^{(i)}(t)\right)-I_{2}^{(i)}(t)+C_{3}^{(i)} t^{2}\right]
\end{aligned}
$$

where,

$$
C_{3}^{(1)}=\frac{5-6 \varepsilon}{1-\varepsilon} \frac{1}{\hat{K}_{1}}, \quad C_{3}^{(3)}=\frac{2(5-4 \varepsilon)}{(1+2 \varepsilon)(2-\varepsilon)} \frac{1}{\hat{K}_{2}}
$$


and,

$$
\begin{aligned}
& I_{21}^{(1)}(t)=\int_{0}^{1} \frac{d p p^{1+\varepsilon}(1-p)^{-1 / 2-\varepsilon}}{\left(1+t^{2} p^{2}\right)^{(1 / 2)}}\left\{1-\varepsilon J^{(1)}(q)\right\}, \\
& I_{31}^{(1)}(t)=\int_{0}^{1} \frac{d p p^{\varepsilon}(1-p)^{-1 / 2-\varepsilon}}{\left(1+t^{2} p^{2}\right)^{1 / 2}}\left\{1-\varepsilon J^{(1)}(y)\right\},
\end{aligned}
$$

and,

$$
\begin{aligned}
& I_{21}^{(3)}(t)=\int_{0}^{1} \frac{d p p^{1+2 \varepsilon}(1-p)^{-1 / 2-\varepsilon}}{\left(1+t^{2} p^{2}\right)^{(1+\varepsilon) / 2}}\left\{1-\frac{\varepsilon}{2} J^{(3)}(q)\right\}, \\
& I_{31}^{(3)}(t)=\int_{0}^{1} \frac{d p p^{2 \varepsilon}(1-p)^{-1 / 2-\varepsilon}}{\left(1+t^{2} p^{2}\right)^{(1+\varepsilon) / 2}}\left\{1-\frac{\varepsilon}{2} J^{(3)}(y)\right\} .
\end{aligned}
$$

The results for $I_{2}^{(2)}(t)$ and $I_{3}^{(2)}(t)$ are,

$$
\begin{aligned}
& \tilde{I}_{2}^{(2)}(t)=\frac{1}{2(1-3 \varepsilon)}\left[(1-4 \varepsilon) I_{1}^{(2)}(t)-t^{2} I_{21}^{(2)}(t)-\frac{2(1-4 \varepsilon) t^{1-\varepsilon}}{(1-\varepsilon)(1+2 \varepsilon)} \frac{1}{\hat{K}_{2}}+2(1-2 \varepsilon) t^{2} I_{31}^{(2)}(t)\right], \\
& \tilde{I}_{3}^{(2)}(t)=\frac{1-2 \varepsilon}{2(2-3 \varepsilon)}\left[2 t^{2}\left(I_{1}^{(2)}(t)-I_{31}^{(2)}(t)\right)-\tilde{I}_{2}^{(2)}(t)\right]
\end{aligned}
$$

where,

$$
\begin{aligned}
& I_{21}^{(2)}(t)=\int_{0}^{1} \frac{d p p^{1+3 \varepsilon}}{(1-p)^{1 / 2+\varepsilon}\left(1+t^{2} p^{2}\right)^{1 / 2+\varepsilon}} J^{(2)}(t p), \\
& I_{31}^{(2)}(t)=\int_{0}^{1} \frac{d p p^{3 \varepsilon}}{(1-p)^{1 / 2+\varepsilon}\left(1+t^{2} p^{2}\right)^{1 / 2+\varepsilon}} J^{(2)}(p) .
\end{aligned}
$$

For integrals $I_{j}^{(1)}(t)$ with $(j=21,31)$ and $(i=1,2,3)$ we have,

$$
\begin{aligned}
I_{j}^{(1)}(t)= & \operatorname{Reg}_{0,1} \sum_{i=0}^{\infty} \frac{\epsilon^{i}}{i !} K_{j} * L_{1}^{i}-\varepsilon \underset{0,1}{\operatorname{Reg}} \sum_{i, j=0}^{\infty} \frac{\epsilon^{i+j}}{i ! j !} K_{j} * L_{3}^{i}\left[K_{3} * L_{2}^{j}\right]_{1}^{x} \\
\left(\frac{t^{2}}{2}\right)^{\varepsilon} I_{1}^{(2)}(t)= & \operatorname{Reg}_{0,1} \sum_{i, j=0}^{\infty} \frac{\epsilon^{i+j}}{i ! j !} K_{j} * \hat{L}_{5}^{i}\left[K_{5} * L_{4}^{j}\right]_{1}^{x} \\
\left(\frac{t}{2}\right)^{\varepsilon} I_{j}^{(3)}(t)= & \operatorname{Reg}_{0,1}^{\infty} \sum_{i=0}^{\infty} \frac{\epsilon^{i}}{i !} K_{j} * \hat{L}_{6}^{i} \\
& -\frac{\varepsilon}{2}\left(\operatorname{Reg}_{0,1} \sum_{i, j=0}^{\infty} \frac{\epsilon^{i+j}}{i ! j !} K_{7} * \hat{L}_{8}^{i}\left[K_{9} * L_{4}^{j}\right]_{1}^{x}+\operatorname{Reg}_{0,1} \sum_{i, j=0}^{\infty} \frac{\epsilon^{i+j}}{i ! j !} K_{7} * \hat{L}_{8}^{i}\left[K_{8} * L_{9}^{j}\right]_{1}^{x}\right)
\end{aligned}
$$

where $K_{i}$ and $L_{i}$ are depth one eMPLs and their definition is provided in appendix E. 
We see that all singularities in $J_{1,1,2}^{r e g}(t)$ and $J_{1,1,1}^{r e g}(t)$, which can be represented as,

$$
\begin{aligned}
J_{1,1,2}^{r e g}(t)= & -\frac{(1-4 \varepsilon) N_{2}}{(1-3 \varepsilon)(1-\varepsilon)(1-2 \varepsilon)(1+2 \varepsilon)}+\bar{J}_{1,1,2}^{r e g}(t) \\
J_{1,1,1}^{r e g}(t)= & -\frac{M^{2} N_{1}}{(1-2 \varepsilon)(2-3 \varepsilon)}\left[\frac{(5-6 \varepsilon)}{\varepsilon(1-\varepsilon)}-\frac{2(5-4 \varepsilon)(2 t)^{\varepsilon}}{\varepsilon(2-\varepsilon)(1+2 \varepsilon)}+\frac{2(1-4 \varepsilon)(2 t)^{\varepsilon-1}}{(1-3 \varepsilon)(1-\varepsilon)(1+2 \varepsilon)}\right] \\
& +\bar{J}_{1,1,1}^{r e g}(t),
\end{aligned}
$$

are exactly cancelled. Indeed, we have,

$$
\bar{J}_{1,1,2}^{\mathrm{reg}}=\frac{\hat{K}_{1} \hat{N}_{1}}{2(1-2 \varepsilon) t^{2}} \tilde{J}_{1,1,2}, \quad \bar{J}_{1,1,1}^{\mathrm{reg}}=-M^{2} \frac{2 \hat{K}_{1} \hat{N}_{1}}{(1-2 \varepsilon)^{2} t^{2}} \tilde{J}_{1,1,1}
$$

and

$$
\begin{aligned}
& \left.\tilde{J}_{1,1,2}(t)=\frac{1}{2(1-3 \varepsilon)}\left\{(1-4 \varepsilon) \hat{J}_{1,2,2}(t)-t^{2} \hat{J}^{(21)}(t)\right)+2(1-2 \varepsilon) t^{2} \hat{J}^{(31)}(t)\right\} \\
& \tilde{J}_{1,1,1}(t)=\frac{(1-2 \varepsilon)}{2(2-3 \varepsilon)}\left\{2 t^{2}\left(\hat{J}_{1,2,2}(t)-\hat{J}^{(31)}(t)\right)-\hat{J}_{1,1,2}(t)\right\}
\end{aligned}
$$

where

$$
\hat{J}^{(j 1)}(t)=\left[\frac{1}{\varepsilon} I_{j 1}^{(1)}(t)+\left(\frac{t^{2}}{2}\right)^{\varepsilon} I_{j 1}^{(2)}(t)-\frac{1}{\varepsilon}\left(\frac{t}{2}\right)^{\varepsilon} I_{j 1}^{(3)}(t)\right] \quad(j=2,3) .
$$

The cancellation of the $1 / \varepsilon$ poles in $\hat{J}^{(j 1)}(t)$ can be shown in the same way as in eq. (4.46).

We conclude this section by remarking that the regularisation procedure described in section 4.2, and applied to the results of eqs. (4.43), (4.62), is meaningful only if the corresponding integral expressions are finite. In our derivations, all integrals $I_{i}^{(m)}(t), \tilde{I}_{j}^{(n)}(t)$ contributing to $J_{122}, J_{112}, J_{111}$, and for which we provide eMPLs expressions, are finite in the $\epsilon \rightarrow 0$ limit. Therefore, the coefficients of their $\epsilon$-expansion are well defined integrals where shuffle regularisation can be safely applied. In order to validate our argument we performed sample analytical and numerical checks. Specifically, we computed the first three $\epsilon$ orders $\left(\epsilon^{i}, i=\{0,1,2,3\}\right)$ of integrals $I_{1, k}^{(i)}, i=\{1,3\}, k=\{1,2\}$ and $I_{1}^{(2)}$ by using the expressions of eq. (4.43), and checked the cancellation of divergent eMPLs at $t=\{0,1\}$ analytically (when possible), and numerically by using the integral definition of eMPLs. Moreover, we compared numerically these results against a direct numerical evaluation of the corresponding integral expressions of eq. (3.6) finding agreement. Finally, by using the same numerical routines, we computed integral $J_{122}$ up to and including $\mathcal{O}\left(\epsilon^{2}\right)$, and found full agreement with a numerical evaluation performed with the computer program FIESTA [113].

\subsection{Example}

We show how the solution strategy of the previous section works in practice by considering integral $I_{1}^{(2)}(t)$. The dependence on the elliptic curve is made explicit by applying the 
variable change (4.25),

$$
I_{1}^{(2)}(t)=\int_{0}^{1} d x \frac{2}{t\left(1-x^{2}\right) y(x)}\left(\frac{\left(1-x^{2}\right)^{3}}{t^{2} x^{2} y(x)^{2}}\right)^{\epsilon} \int_{0}^{t\left(1-x^{2}\right)} d z \frac{1}{\sqrt{z^{2}+1}}\left(z+\frac{1}{z}\right)^{\epsilon}
$$

By applying eq. (4.35) the inner integral can be expressed as

$$
\int_{0}^{t-t x^{2}} d z \frac{1}{\sqrt{z^{2}+1}}\left(z+\frac{1}{z}\right)^{\epsilon}=-\int_{1}^{x} d z \frac{2 z}{y(z)}\left(\frac{t y^{2}(z)}{1-z^{2}}\right)^{\epsilon} .
$$

All the $\epsilon$-powers can be expanded in $\epsilon$ by eq. (4.30). For example we have

$$
\left(\frac{t y^{2}(x)}{1-x^{2}}\right)^{\epsilon}=\sum_{i=0}^{\infty} \frac{\epsilon^{i}}{i !} \log ^{i}\left(\frac{t y^{2}(x)}{1-x^{2}}\right)
$$

The resulting logarithm can be expressed in terms of eMPLs by eq. (4.31),

$$
\log \left(\frac{t y^{2}(x)}{1-x^{2}}\right)=\log \left(t^{2}+1\right)-\log (t)+\int_{0}^{x} d z \frac{2 z\left(t^{2}\left(z^{2}-1\right)^{2}-1\right)}{t^{2}\left(z^{2}-1\right) y(z)^{2}} .
$$

The integrand above can be written in terms of the integration kernels as,

$$
\frac{2 z\left(t^{2}\left(z^{2}-1\right)^{2}-1\right)}{t^{2}\left(z^{2}-1\right) y(z)^{2}}=\sum_{i=1}^{4} \varphi_{1}\left(a_{i}, z\right)-\varphi_{1}(-1, z)-\varphi_{1}(1, z),
$$

where we denoted with $a_{i}$ the four roots of the elliptic curve,

$$
a_{1}=-\frac{\sqrt{t-i}}{\sqrt{t}}, a_{2}=\frac{\sqrt{t-i}}{\sqrt{t}}, a_{3}=-\frac{\sqrt{t+i}}{\sqrt{t}}, a_{4}=\frac{\sqrt{t+i}}{\sqrt{t}} .
$$

Upon integration we find,

$$
L_{4} \equiv \log \left(\frac{t y^{2}(x)}{1-x^{2}}\right)=\sum_{i=1}^{4} E_{4}\left(\underset{a_{i}}{1} ; x\right)-E_{4}(\underset{-1}{1} ; x)-E_{4}(\underset{1}{1} ; x)+\log \left(t^{2}+1\right)-\log (t) .
$$

Finally, all prefactors can be expressed in terms of integration kernels, for example, referring to eq. (4.69),

$$
\frac{2}{t\left(1-x^{2}\right) y(x)}=\varphi_{-1}(-1, x)-\varphi_{-1}(1, x)-\varphi_{1}(1, x) .
$$

By taking the primitive of eq. (4.76) we obtain,

$$
K_{4} \equiv E_{4}\left({ }_{-1}^{-1} ; x\right)-E_{4}\left(\begin{array}{c}
-1 \\
1
\end{array} ; x\right)-E_{4}\left(\begin{array}{c}
1 \\
1
\end{array} ; x\right) .
$$

By applying these methods to all relevant logarithms and prefactors, we obtain a result in terms of integrals of the form (4.37), which are directly evaluated to eMPLs by, e.g., eq. (4.39),

$$
I_{1}^{(2)}(t)=\underset{0,1}{\operatorname{Reg}} \sum_{i, j=0}^{\infty} \frac{\epsilon^{i+j}}{i ! j !} K_{4} * L_{5}^{i}\left[K_{5} * L_{4}^{j}\right]_{1}^{x}
$$

where,

$$
\begin{aligned}
& L_{5}=-\sum_{i=1}^{4} E_{4}\left(\underset{a_{i}}{1} ; x\right)+3 E_{4}(\underset{-1}{1} ; x)-2 E_{4}(\underset{0}{1} ; x)+3 E_{4}(\underset{1}{1} ; x)-\log \left(t^{2}+1\right), \\
& K_{5}=-2 E_{4}(\underset{\infty}{-1} ; x) .
\end{aligned}
$$




\section{Conclusions}

In this paper we studied a family of sunrise integrals with two different internal masses and pseudo-threshold kinematics in dimensional regularisation. These integrals admit a closedform solution in terms of hypergeometric functions [71] and we use this representation as the starting point of our analysis. Specifically, we show that all relevant hypergeometric functions admit a representation in terms of double iterated integrals depending on one elliptic curve and no other algebraic functions. When expanding these integrals with respect to the (vanishing) dimensional regulator, the coefficients of the expansion are iterated integrals over rational functions on the relevant elliptic curve. We derive an expression for the relevant sunrise integrals valid to all orders of the dimensional regulator in terms of eMPLs.

\section{A Integral representations for the hypergeometric functions}

In this section we provide the derivation of the integral representations for the ${ }_{4} F_{3}$-hypergeometric functions of eq. (2.2), identities eq. (3.3). Similar techniques have been used in refs. $[6,56,114-118]$. In what follows we use the following definitions and identities,

$$
\hat{K}_{1}=\frac{\Gamma\left(\frac{1}{2}\right)}{\Gamma\left(\frac{1}{2}-\epsilon\right) \Gamma(\epsilon+1)}=\frac{\hat{K}}{2^{2 \varepsilon}}, \quad \hat{K}_{2}=\frac{\Gamma\left(\epsilon+\frac{1}{2}\right)}{\Gamma\left(\frac{1}{2}-\epsilon\right) \Gamma(2 \epsilon+1)}=\frac{\hat{K}_{1}}{2^{2 \varepsilon}}=\frac{\hat{K}}{2^{4 \varepsilon}},
$$

where $\hat{K}$ is defined in eq. (3.4). We present a detailed derivation only for diagram $J_{1,2,2}$. For diagrams $J_{1,1,2}$ and $J_{1,1,1}$ we present only the final results as their derivation follows steps similar to those of $J_{1,2,2}$.

The first type of hypergeometric functions. The first ${ }_{4} F_{3}$-hypergeometric function of $J_{1,2,2}$ admits the following series representation,

$$
\begin{aligned}
F_{1}^{(1)}(t) & \equiv{ }_{4} F_{3}\left(\begin{array}{c}
1, \frac{3}{2}, 1+\frac{\varepsilon}{2}, \frac{3}{2}+\frac{\varepsilon}{2} \\
2-\varepsilon, \frac{5}{4}, \frac{7}{4}
\end{array} \mid-t^{2}\right), \\
& =\sum_{m=0}^{\infty} \frac{\Gamma\left(m+\frac{3}{2}\right) \Gamma\left(m+1+\frac{\varepsilon}{2}\right) \Gamma\left(m+\frac{3}{2}+\frac{\varepsilon}{2}\right)}{\Gamma(m+2-\varepsilon) \Gamma\left(m+\frac{5}{4}\right) \Gamma\left(m+\frac{7}{4}\right)} \frac{\Gamma(2-\varepsilon) \Gamma\left(\frac{5}{4}\right) \Gamma\left(\frac{7}{4}\right)}{\Gamma\left(\frac{3}{2}\right) \Gamma\left(1+\frac{\varepsilon}{2}\right) \Gamma\left(\frac{3}{2}+\frac{\varepsilon}{2}\right)}\left(-t^{2}\right)^{m},
\end{aligned}
$$

where $t=m^{2} /\left(2 M^{2}\right)$ as defined in eq. (3.1) of the main text. The product $\Gamma(\alpha) \Gamma(1 / 2+\alpha)$ can be written as,

$$
\Gamma(\alpha) \Gamma(1 / 2+\alpha)=2^{1-2 \alpha} \sqrt{\pi} \Gamma(2 \alpha),
$$

which results in the following simplified expression for $F_{1}(t)$,

$$
\sum_{m=0}^{\infty} \frac{\Gamma\left(m+\frac{3}{2}\right) \Gamma(2 m+2+\varepsilon)}{\Gamma(m+2-\varepsilon) \Gamma\left(2 m+\frac{5}{2}\right)} \frac{\Gamma(2-\varepsilon) \Gamma\left(\frac{5}{2}\right)}{\Gamma\left(\frac{3}{2}\right) \Gamma(2+\varepsilon)}\left(-t^{2}\right)^{m} .
$$


It is convenient to use the following integral representations for the ratio of gamma functions,

$$
\frac{\Gamma(2 m+2+\varepsilon)}{\Gamma\left(2 m+\frac{5}{2}\right)}=\int_{0}^{1} d p \frac{p^{2 m+1+\varepsilon}(1-p)^{-1 / 2-\varepsilon}}{\Gamma\left(\frac{1}{2}-\varepsilon\right)} .
$$

We find,

$$
F_{1}(t)=\int_{0}^{1} d p \frac{p^{1+\varepsilon}(1-p)^{-1 / 2-\varepsilon}}{\Gamma\left(\frac{1}{2}-\varepsilon\right)} \sum_{m=0}^{\infty} \frac{\Gamma\left(m+\frac{3}{2}\right)}{\Gamma(m+2-\varepsilon)} \frac{\Gamma(2-\varepsilon) \Gamma\left(\frac{5}{2}\right)}{\Gamma\left(\frac{3}{2}\right) \Gamma(2+\varepsilon)}\left(-(t p)^{2}\right)^{m} .
$$

In order to proceed with our analysis it is convenient to consider first the series on the right hand side in the limit $\varepsilon=0$,

$$
\sum_{m=0}^{\infty} \frac{\Gamma\left(m+\frac{3}{2}\right)}{(m+1) !}\left(-(t p)^{2}\right)^{m}=\sum_{m=1}^{\infty} \frac{\Gamma\left(m+\frac{1}{2}\right)}{m !}\left(-(t p)^{2}\right)^{m-1}=-\frac{\Gamma\left(\frac{1}{2}\right)}{(t p)^{2}}\left[\frac{1}{\left(1+t^{2} p^{2}\right)^{1 / 2}}-1\right] .
$$

In the general case we have,

$$
\begin{aligned}
\sum_{m=0}^{\infty} \frac{\Gamma\left(m+\frac{3}{2}\right)}{\Gamma(m+2-\varepsilon)}\left(-(t p)^{2}\right)^{m} & =\sum_{m=1}^{\infty} \frac{\Gamma\left(m+\frac{1}{2}\right)}{\Gamma(m+1-\varepsilon)}\left(-(t p)^{2}\right)^{m-1} \\
& =-\frac{\Gamma\left(\frac{1}{2}\right)}{\Gamma(1-\varepsilon)(t p)^{2}}\left[{ }_{2} F_{1}\left(1, \frac{1}{2} ; 1-\varepsilon ;-p^{2} t^{2}\right)-1\right] .
\end{aligned}
$$

Using standard properties of the ${ }_{2} F_{1}$-function,

$$
{ }_{2} F_{1}(a, b ; c ; z)=(1-z)_{2}^{b} F_{1}\left(c-a, b ; c ; \frac{z-1}{z}\right),
$$

we obtain,

$$
{ }_{2} F_{1}\left(1, \frac{1}{2} ; 1-\varepsilon ;-p^{2} t^{2}\right)=\frac{1}{\left(1+t^{2} p^{2}\right)^{1 / 2}}{ }_{2} F_{1}\left(\frac{1}{2},-\varepsilon ; 1-\varepsilon ; q(p)\right),
$$

where $q$ was defined in eq. (3.8) of the main text, and in what follows we suppress the $p$-dependence of $q(p)$ for ease of notation. The last ${ }_{2} F_{1}$-function admits the following representation,

$$
{ }_{2} F_{1}\left(\frac{1}{2},-\varepsilon ; 1-\varepsilon ; q\right)=\sum_{m=0}^{\infty} \frac{\Gamma\left(m+\frac{1}{2}\right)}{m ! \Gamma\left(\frac{1}{2}\right)} \frac{-\varepsilon}{m-\varepsilon} q^{m}=1-\varepsilon \sum_{m=1}^{\infty} \frac{\Gamma\left(m+\frac{1}{2}\right)}{m ! \Gamma\left(\frac{1}{2}\right)} \frac{1}{m-\varepsilon} q^{m} .
$$

Using the integral representation for the factor $1 /(m-\varepsilon)=\int_{0}^{1} d z z^{m-1-\varepsilon}$, we have,

$$
{ }_{2} F_{1}\left(\frac{1}{2},-\varepsilon ; 1-\varepsilon ; q\right)=1-\varepsilon \int_{0}^{1} \frac{d z}{z^{1+\varepsilon}}\left[\frac{1}{\sqrt{1-z q}}-1\right]=1-\varepsilon \int_{0}^{q} \frac{d z_{1} q^{\varepsilon}}{z_{1}^{1+\varepsilon}}\left[\frac{1}{\sqrt{1-z_{1}}}-1\right] .
$$

Combining these results, eq. (A.8) can be written as,

$$
\sum_{m=0}^{\infty} \frac{\Gamma\left(m+\frac{3}{2}\right)}{\Gamma(m+2-\varepsilon)}\left(-(t p)^{2}\right)^{m}=-\frac{\Gamma\left(\frac{1}{2}\right)}{\Gamma(1-\varepsilon)(t p)^{2}} f_{1}^{(1)}(p t),
$$


where

$$
f_{1}^{(1)}(p t)=\frac{1}{\left(1+t^{2} p^{2}\right)^{1 / 2}}\left\{1-\varepsilon J^{(1)}(q)\right\}-1
$$

and $J^{(1)}(q)$ is defined in eq. (3.7).

The final result for $F_{1}^{(1)}(t)$ reads,

$$
F_{1}^{(1)}(t)=-\frac{3(1-\varepsilon)}{2(1+\varepsilon) t^{2}} \hat{K}_{1} I_{1}^{(1)}(t), \quad I_{1}^{(1)}(t)=\int_{0}^{1} d p p^{\varepsilon-1}(1-p)^{-1 / 2-\varepsilon} f_{1}^{(1)}(p t),
$$

where the normalization $\hat{K}_{1}$ was determined in eq. (A.1). We note that the elliptic structure of eq. (A.15) is carried by the product $(1-p)^{-1 / 2-\varepsilon}\left(1+t^{2} p^{2}\right)^{-1 / 2}$.

The first ${ }_{4} F_{3}$-hypergeometric functions of $J_{1,1,2}$ and $J_{1,1,1}, F_{2}^{(1)}(t)$ and $F_{3}^{(1)}(t)$, admit the following series representations,

$$
\begin{aligned}
F_{2}^{(1)}(t) & \equiv{ }_{4} F_{3}\left(\begin{array}{c}
1, \frac{1}{2}, 1+\frac{\varepsilon}{2}, \frac{1}{2}+\frac{\varepsilon}{2} \\
2-\varepsilon, \frac{3}{4}, \frac{5}{4}
\end{array} \mid-t^{2}\right) \\
& =\sum_{m=0}^{\infty} \frac{\Gamma\left(m+\frac{1}{2}\right) \Gamma\left(m+1+\frac{\varepsilon}{2}\right) \Gamma\left(m+\frac{1}{2}+\frac{\varepsilon}{2}\right)}{\Gamma(m+2-\varepsilon) \Gamma\left(m+\frac{3}{4}\right) \Gamma\left(m+\frac{5}{4}\right)} \frac{\Gamma(2-\varepsilon) \Gamma\left(\frac{3}{4}\right) \Gamma\left(\frac{5}{4}\right)}{\Gamma\left(\frac{1}{2}\right) \Gamma\left(1+\frac{\varepsilon}{2}\right) \Gamma\left(\frac{1}{2}+\frac{\varepsilon}{2}\right)}\left(-t^{2}\right)^{m}, \\
F_{3}^{(1)}(t) & \equiv{ }_{4} F_{3}\left(\begin{array}{c}
1, \frac{1}{2}, \frac{\varepsilon}{2}, \frac{1}{2}+\frac{\varepsilon}{2} \\
2-\varepsilon, \frac{3}{4}, \frac{5}{4}
\end{array} \mid-t^{2}\right) \\
& =\sum_{m=0}^{\infty} \frac{\Gamma\left(m+\frac{1}{2}\right) \Gamma\left(m+\frac{\varepsilon}{2}\right) \Gamma\left(m+\frac{1}{2}+\frac{\varepsilon}{2}\right)}{\Gamma(m+2-\varepsilon) \Gamma\left(m+\frac{3}{4}\right) \Gamma\left(m+\frac{5}{4}\right)} \frac{\Gamma(2-\varepsilon) \Gamma\left(\frac{3}{4}\right) \Gamma\left(\frac{5}{4}\right)}{\Gamma\left(\frac{1}{2}\right) \Gamma\left(\frac{\varepsilon}{2}\right) \Gamma\left(\frac{1}{2}+\frac{\varepsilon}{2}\right)}\left(-t^{2}\right)^{m} .
\end{aligned}
$$

In a similar way we obtain,

$$
\begin{aligned}
& F_{2}^{(1)}(t)=\frac{(1-\varepsilon)}{t^{2}} \hat{K}_{1} \int_{0}^{1} d p p^{\varepsilon-2}(1-p)^{-1 / 2-\varepsilon}\left[\left(1+t^{2} p^{2}\right)^{1 / 2}\left\{1-\varepsilon J_{2}^{(1)}(q)\right\}-1\right], \\
& F_{3}^{(1)}(t)=\frac{2 \varepsilon(1-\varepsilon)}{(1-2 \varepsilon) t^{2}} \hat{K}_{1} \int_{0}^{1} d p p^{\varepsilon-3}(1-p)^{1 / 2-\varepsilon}\left[\left(1+t^{2} p^{2}\right)^{1 / 2}\left\{1-\varepsilon J_{2}^{(1)}(q)\right\}-1\right],
\end{aligned}
$$

where

$$
J_{2}^{(1)}(q)=q^{\epsilon} \int_{0}^{q} d z\left((1-z)^{\frac{1}{2}}-1\right) z^{-\epsilon-1} .
$$

The second type of the hypergeometric functions. The second ${ }_{4} F_{3}$-hypergeometric function of $J_{1,2,2}$ admits the following series representation,

$$
\begin{aligned}
F_{1}^{(2)}(t) & \equiv{ }_{4} F_{3}\left(\begin{array}{c}
1, \frac{1}{2}+\varepsilon, 1+\frac{\varepsilon}{2}, 1+\varepsilon \\
\frac{3}{2}-\frac{\varepsilon}{2}, \frac{3}{4}+\frac{\varepsilon}{2}, \frac{5}{4}+\frac{\varepsilon}{2}
\end{array} \mid-t^{2}\right) \\
& =\sum_{m=0}^{\infty} \frac{\Gamma\left(m+\frac{1}{2}+\varepsilon\right) \Gamma\left(m+1+\frac{\varepsilon}{2}\right) \Gamma(m+1+\varepsilon)}{\Gamma\left(m+\frac{3}{2}-\frac{\varepsilon}{2}\right) \Gamma\left(m+\frac{3}{4}+\frac{\varepsilon}{2}\right) \Gamma\left(m+\frac{5}{4}+\frac{\varepsilon}{2}\right)} \frac{\Gamma\left(\frac{3}{2}-\frac{\varepsilon}{2}\right) \Gamma\left(\frac{3}{4}+\frac{\varepsilon}{2}\right) \Gamma\left(\frac{5}{4}+\frac{\varepsilon}{2}\right)}{\Gamma\left(\frac{1}{2}+\varepsilon\right) \Gamma\left(1+\frac{\varepsilon}{2}\right) \Gamma(1+\varepsilon)}\left(-t^{2}\right)^{m} .
\end{aligned}
$$

As in the previous section, we express $F_{2}(t)$ as,

$$
\sum_{m=0}^{\infty} \frac{\Gamma\left(m+1+\frac{\varepsilon}{2}\right) \Gamma(2 m+2+2 \varepsilon)}{\Gamma\left(m+\frac{3}{2}-\frac{\varepsilon}{2}\right) \Gamma\left(2 m+\frac{3}{2}+\varepsilon\right)} \frac{\Gamma\left(\frac{3}{2}-\frac{\varepsilon}{2}\right) \Gamma\left(\frac{1}{2}+\varepsilon\right)}{\Gamma\left(1+\frac{\varepsilon}{2}\right) \Gamma(1+2 \varepsilon)}\left(-t^{2}\right)^{m},
$$


where we used the standard identity,

$$
{ }_{2} F_{1}(a, b ; c ; z)=(1-z)^{c-b-a}{ }_{2} F_{1}(c-a, c-b ; c ; z) .
$$

By turning to integral representations for gamma functions,

$$
\frac{\Gamma(2 m+1+2 \varepsilon)}{\Gamma\left(2 m+\frac{3}{2}+\varepsilon\right)}=\int_{0}^{1} d p \frac{p^{2 m+2 \varepsilon}(1-p)^{-1 / 2-\varepsilon}}{\Gamma\left(\frac{1}{2}-\varepsilon\right)},
$$

we find,

$$
F_{1}^{(2)}(t)=\int_{0}^{1} d p \frac{p^{2 \varepsilon}(1-p)^{-1 / 2-\varepsilon}}{\Gamma\left(\frac{1}{2}-\varepsilon\right)} \sum_{m=0}^{\infty} \frac{\Gamma\left(m+1+\frac{\varepsilon}{2}\right)}{\Gamma\left(m+\frac{3}{2}-\frac{\varepsilon}{2}\right)} \frac{\Gamma\left(\frac{3}{2}-\frac{\varepsilon}{2}\right) \Gamma\left(\frac{3}{2}+\varepsilon\right)}{\Gamma\left(+\frac{\varepsilon}{2}\right) \Gamma(1+2 \varepsilon)}\left(-(t p)^{2}\right)^{m}
$$

As before, we consider first the $\varepsilon=0$ limit,

$$
\begin{gathered}
\sum_{m=0}^{\infty} \frac{\Gamma(m+1) \Gamma\left(\frac{1}{2}\right)}{\Gamma\left(m+\frac{3}{2}\right)}\left(-t^{2}\right)^{m}=2{ }_{2} F_{1}\left(1,1 ; \frac{3}{2} ;-t^{2}\right)=\frac{2}{\left(1+t^{2}\right)^{1 / 2}}{ }_{2} F_{1}\left(\frac{1}{2}, \frac{1}{2} ; \frac{1}{2} ;-t^{2}\right) \\
=\frac{1}{\left(1+t^{2}\right)^{1 / 2}} \sum_{m=0}^{\infty} \frac{\Gamma\left(m+\frac{1}{2}\right)}{m ! \Gamma\left(\frac{1}{2}\right)} \frac{1}{m+\frac{1}{2}}\left(-t^{2}\right)^{m}
\end{gathered}
$$

By taking $1 /(m+1 / 2)=\int_{0}^{1} d z z^{m-1 / 2}$, we find $\left(z=s^{2}\right)$,

$$
\begin{gathered}
\frac{1}{\left(1+t^{2}\right)^{1 / 2}} \sum_{m=0}^{\infty} \frac{\Gamma\left(m+\frac{1}{2}\right)}{m ! \Gamma\left(\frac{1}{2}\right)} \frac{1}{m+\frac{1}{2}}\left(-t^{2}\right)^{m}=\frac{1}{\left(1+t^{2}\right)^{1 / 2}} \int_{0}^{1} \frac{d z}{z} \frac{1}{\sqrt{1+z t^{2}}} \\
=\frac{2}{\left(1+t^{2}\right)^{1 / 2}} \int_{0}^{1} \frac{d s}{\sqrt{1+s^{2} t^{2}}}=\frac{1}{t \sqrt{1+t^{2}}} \log \frac{\sqrt{1+t^{2}}+1}{\sqrt{1+t^{2}}-1} .
\end{gathered}
$$

In the general case,

$$
\begin{aligned}
\sum_{m=0}^{\infty} & \frac{\Gamma\left(m+1+\frac{\varepsilon}{2}\right) \Gamma\left(\frac{3}{2}-\frac{\varepsilon}{2}\right)}{\Gamma\left(m+\frac{3}{2}-\frac{\varepsilon}{2}\right) \Gamma\left(1+\frac{\varepsilon}{2}\right)}\left(-t^{2}\right)^{m} \\
& ={ }_{2} F_{1}\left(1+\frac{\varepsilon}{2}, 1 ; \frac{3}{2}-\frac{\varepsilon}{2} ;-t^{2}\right)=\frac{1}{\left(1+t^{2}\right)^{1 / 2+\varepsilon}}{ }_{2} F_{1}\left(\frac{1}{2}-\varepsilon, \frac{1}{2}-\frac{\varepsilon}{2} ; \frac{3}{2}-\frac{\varepsilon}{2} ;-t^{2}\right) \\
& =\frac{1}{\left(1+t^{2}\right)^{1 / 2+\varepsilon}} \sum_{m=0}^{\infty} \frac{\Gamma\left(m+\frac{1}{2}-\varepsilon\right)}{m ! \Gamma\left(\frac{1}{2}-\varepsilon\right)} \frac{\left(\frac{1}{2}-\frac{\varepsilon}{2}\right)}{m+\frac{1}{2}-\frac{\varepsilon}{2}}\left(-t^{2}\right)^{m}
\end{aligned}
$$

and by taking $1 /(m+1 / 2-\varepsilon / 2)=\int_{0}^{1} d z z^{m-1 / 2-\varepsilon / 2}$, we have $\left(z=s^{2}\right)$,

$$
\begin{gathered}
\frac{1-\varepsilon}{2\left(1+t^{2}\right)^{1 / 2+\varepsilon}} \sum_{m=0}^{\infty} \frac{\Gamma\left(m+\frac{1}{2}-\varepsilon\right)}{m ! \Gamma\left(\frac{1}{2}-\varepsilon\right)} \frac{1}{m+\frac{1}{2}-\frac{\varepsilon}{2}}\left(-t^{2}\right)^{m}=\frac{1-\varepsilon}{2\left(1+t^{2}\right)^{1 / 2+\varepsilon}} \int_{0}^{1} \frac{d z}{z^{\frac{1}{2}+\frac{\varepsilon}{2}}} \frac{1}{\left(1+z t^{2}\right)^{\frac{1}{2}-\varepsilon}} \\
=\frac{1-\varepsilon}{\left(1+t^{2}\right)^{1 / 2+\varepsilon}} \int_{0}^{1} \frac{d s}{s^{\varepsilon}} \frac{1}{\left(1+s^{2} t^{2}\right)^{\frac{1}{2}-\varepsilon}} \equiv \frac{1-\varepsilon}{\left(1+t^{2}\right)^{1 / 2+\varepsilon} t^{1-\varepsilon}} J^{(2)}(t),
\end{gathered}
$$

where $J^{(2)}(t)$ is defined in eq. (3.7). 
The final results for $F_{1}^{(2)}(t)$ reads,

$$
F_{1}^{(2)}(t)=\frac{(1-\varepsilon)(1+2 \varepsilon)}{2^{2} t^{1-\varepsilon}} \hat{K}_{2} I_{1}^{(2)}(t), \quad I_{1}^{(2)}(t)=\int_{0}^{1} d p p^{3 \varepsilon-1}(1-p)^{-1 / 2-\varepsilon} f_{1}^{(2)}(p t),
$$

where

$$
f_{1}^{(2)}(p t)=\frac{1}{\left(1+t^{2} p^{2}\right)^{1 / 2+\varepsilon}} J^{(2)}(p t)
$$

and $J^{(2)}(y)$ is defined in (3.7). The normalization $\hat{K}_{2}$ is defined in eq. (A.1).

The second ${ }_{4} F_{3}$-hypergeometric functions of $J_{1,1,2}$ and $J_{1,1,2}$ admit the following series representation,

$$
\begin{aligned}
F_{2}^{(2)}(t) & \equiv{ }_{4} F_{3}\left(\begin{array}{c}
1, \frac{1}{2}+\varepsilon, \frac{\varepsilon}{2}, \varepsilon \\
\frac{3}{2}-\frac{\varepsilon}{2}, \frac{1}{4}+\frac{\varepsilon}{2}, \frac{3}{4}+\frac{\varepsilon}{2}
\end{array} \mid-t^{2}\right) \\
& =\sum_{m=0}^{\infty} \frac{\Gamma\left(m+\frac{1}{2}+\varepsilon\right) \Gamma\left(m+\frac{\varepsilon}{2}\right) \Gamma(m+\varepsilon)}{\Gamma\left(m+\frac{3}{2}-\frac{\varepsilon}{2}\right) \Gamma\left(m+\frac{1}{4}+\frac{\varepsilon}{2}\right) \Gamma\left(m+\frac{3}{4}+\frac{\varepsilon}{2}\right)} \frac{\Gamma\left(\frac{3}{2}-\frac{\varepsilon}{2}\right) \Gamma\left(\frac{1}{4}+\frac{\varepsilon}{2}\right) \Gamma\left(\frac{3}{4}+\frac{\varepsilon}{2}\right)}{\Gamma\left(\frac{1}{2}+\varepsilon\right) \Gamma\left(\frac{\varepsilon}{2}\right) \Gamma(\varepsilon)}\left(-t^{2}\right)^{m} \\
F_{3}^{(2)}(t) & \equiv{ }_{4} F_{3}\left(\begin{array}{c}
1,-\frac{1}{2}+\varepsilon, \frac{\varepsilon}{2}, \varepsilon \\
\frac{3}{2}-\frac{\varepsilon}{2}, \frac{1}{4}+\frac{\varepsilon}{2}, \frac{3}{4}+\frac{\varepsilon}{2}
\end{array} \mid-t^{2}\right) \\
& =\sum_{m=0}^{\infty} \frac{\Gamma\left(m-\frac{1}{2}+\varepsilon\right) \Gamma\left(m+\frac{\varepsilon}{2}\right) \Gamma(m+\varepsilon)}{\Gamma\left(m+\frac{3}{2}-\frac{\varepsilon}{2}\right) \Gamma\left(m+\frac{1}{4}+\frac{\varepsilon}{2}\right) \Gamma\left(m+\frac{3}{4}+\frac{\varepsilon}{2}\right)} \frac{\Gamma\left(\frac{3}{2}-\frac{\varepsilon}{2}\right) \Gamma\left(\frac{1}{4}+\frac{\varepsilon}{2}\right) \Gamma\left(\frac{3}{4}+\frac{\varepsilon}{2}\right)}{\Gamma\left(-\frac{1}{2}+\varepsilon\right) \Gamma\left(\frac{\varepsilon}{2}\right) \Gamma(\varepsilon)}\left(-t^{2}\right)^{m}
\end{aligned}
$$

Splitting the cases $m=0$ and $m \neq 0$, we have:

$$
\begin{aligned}
& F_{2}^{(2)}(t)=\frac{(1-\varepsilon)}{(1-2 \varepsilon)}\left[1-\frac{2 \varepsilon^{2} \hat{K}_{2}}{t^{1-\varepsilon}} I_{2}^{(2)}(t)\right]=1-\frac{(1-\varepsilon)}{(1-2 \varepsilon)} \frac{2 \varepsilon^{2} \hat{K}_{2}}{t^{1-\varepsilon}} \tilde{I}_{2}^{(2)}(t) \\
& I_{2}^{(2)}(t)=\int_{0}^{1} d p \frac{p^{3 \varepsilon-2}}{(1-p)^{1 / 2+\varepsilon}} f_{2}^{(2)}(p t), \quad \tilde{I}_{2}^{(2)}(t)=\int_{0}^{1} d p \frac{p^{3 \varepsilon-2}}{(1-p)^{1 / 2+\varepsilon}} \tilde{f}_{2}^{(2)}(p t) \\
& F_{3}^{(2)}(t)=1+\frac{4 \varepsilon^{2}(1-\varepsilon) \hat{K}_{2}}{(1-2 \varepsilon) t^{1-\varepsilon}} \tilde{I}_{3}^{(2)}(t), \quad \tilde{I}_{3}^{(2)}(t)=\int_{0}^{1} d p \frac{p^{3 \varepsilon-3}}{(1-p)^{-1 / 2+\varepsilon}} \tilde{f}_{2}^{(2)}(p t)
\end{aligned}
$$

where

$$
f_{2}^{(2)}(p t)=\left(1+t^{2} p^{2}\right)^{1 / 2-\varepsilon} J^{(2)}(p t), \quad \tilde{f}_{2}^{(2)}(p t)=f_{2}^{(2)}(p t)-\frac{(p t)^{1-\varepsilon}}{1-\varepsilon} .
$$

The form $\tilde{f}_{2}^{(2)}(p t)$ is conveninet at small $p$ behaviour, because it is $\sim O\left(p^{3-\varepsilon}\right)$. Indeed, st small $t$, the integral $\int_{0}^{1} d s /\left[s^{\varepsilon}\left(1+s^{2} t^{2}\right)^{\frac{1}{2}-\varepsilon}\right]$ has the following form

$$
\int_{0}^{1} \frac{d s}{s^{\varepsilon}} \frac{1}{\left(1+s^{2} t^{2}\right)^{\frac{1}{2}-\varepsilon}} \approx \frac{1}{(1-\varepsilon)}+O\left(t^{2}\right)
$$

and, thus,

$$
\tilde{J}^{(2)}(p t)=J^{(2)}(p t)-\frac{1}{\left(1+t^{2} p^{2}\right)^{1 / 2-\varepsilon}} \frac{(p t)^{1-\varepsilon}}{(1-\varepsilon)} \sim O\left((p t)^{3-\varepsilon}\right) .
$$


The third type of hypergeometric functions. The third ${ }_{4} F_{3}$-hypergeometric function admits the following series representation,

$$
\begin{aligned}
F_{1}^{(3)}(t) & \equiv{ }_{4} F_{3}\left(\begin{array}{c}
1, \frac{3}{2}+\frac{\varepsilon}{2}, 1+\varepsilon, \frac{3}{2}+\varepsilon \\
2-\frac{\varepsilon}{2}, \frac{5}{4}+\frac{\varepsilon}{2}, \frac{7}{4}+\frac{\varepsilon}{2}
\end{array} \mid-t^{2}\right) \\
& =\sum_{m=0}^{\infty} \frac{\Gamma\left(m+\frac{3}{2}+\frac{\varepsilon}{2}\right) \Gamma\left(m+\frac{3}{2}+\varepsilon\right) \Gamma(m+1+\varepsilon)}{\Gamma\left(m+2-\frac{\varepsilon}{2}\right) \Gamma\left(m+\frac{5}{4}+\frac{\varepsilon}{2}\right) \Gamma\left(m+\frac{7}{4}+\frac{\varepsilon}{2}\right)} \frac{\Gamma\left(2-\frac{\varepsilon}{2}\right) \Gamma\left(\frac{5}{4}+\frac{\varepsilon}{2}\right) \Gamma\left(\frac{7}{4}+\frac{\varepsilon}{2}\right)}{\Gamma\left(\frac{3}{2}+\frac{\varepsilon}{2}\right) \Gamma(1+\varepsilon) \Gamma\left(\frac{3}{2}+\varepsilon\right)}\left(-t^{2}\right)^{m} .
\end{aligned}
$$

The previous sum simplifies to,

$$
\sum_{m=0}^{\infty} \frac{\Gamma\left(m+1+\frac{\varepsilon}{2}\right) \Gamma(2 m+2+2 \varepsilon)}{\Gamma\left(m+\frac{3}{2}-\frac{\varepsilon}{2}\right) \Gamma\left(2 m+\frac{3}{2}+\varepsilon\right)} \frac{\Gamma\left(\frac{3}{2}-\frac{\varepsilon}{2}\right) \Gamma\left(\frac{1}{2}+\varepsilon\right)}{\Gamma\left(1+\frac{\varepsilon}{2}\right) \Gamma(1+2 \varepsilon)}\left(-t^{2}\right)^{m} .
$$

By considering the following representations for ratios of gamma functions,

$$
\frac{\Gamma(2 m+2+2 \varepsilon)}{\Gamma\left(2 m+\frac{5}{2}+\varepsilon\right)}=\int_{0}^{1} d p \frac{p^{2 m+1+2 \varepsilon}(1-p)^{-1 / 2-\varepsilon}}{\Gamma\left(\frac{1}{2}-\varepsilon\right)},
$$

we find,

$$
F_{1}^{(3)}(t)=\int_{0}^{1} d p \frac{p^{1+2 \varepsilon}(1-p)^{-1 / 2-\varepsilon}}{\Gamma\left(\frac{1}{2}-\varepsilon\right)} \sum_{m=0}^{\infty} \frac{\Gamma\left(m+\frac{3}{2}+\frac{\varepsilon}{2}\right)}{\Gamma\left(m+2-\frac{\varepsilon}{2}\right)} \frac{\Gamma\left(2-\frac{\varepsilon}{2}\right) \Gamma\left(\frac{5}{2}+\varepsilon\right)}{\Gamma\left(\frac{3}{2}+\frac{\varepsilon}{2}\right) \Gamma(2+2 \varepsilon)}\left(-(t p)^{2}\right)^{m} .
$$

As in the previous sections, we consider first the limit of the right hand side of the previous equation, which is equivalent to eq. (A.7). In the general case,

$$
\begin{aligned}
\sum_{m=0}^{\infty} \frac{\Gamma\left(m+\frac{3}{2}+\frac{\varepsilon}{2}\right)}{\Gamma\left(m+2-\frac{\varepsilon}{2}\right)}\left(-(t p)^{2}\right)^{m} & =\sum_{m=1}^{\infty} \frac{\Gamma\left(m+\frac{1}{2}+\frac{\varepsilon}{2}\right)}{\Gamma\left(m+1-\frac{\varepsilon}{2}\right)}\left(-(t p)^{2}\right)^{m-1} \\
& =-\frac{\Gamma\left(\frac{1}{2}+\frac{\varepsilon}{2}\right)}{\Gamma\left(1-\frac{\varepsilon}{2}\right)(t p)^{2}}\left[{ }_{2} F_{1}\left(1, \frac{1}{2}+\frac{\varepsilon}{2} ; 1-\frac{\varepsilon}{2} ;-p^{2} t^{2}\right)-1\right] .
\end{aligned}
$$

By using eq. (A.9) we obtain,

$$
{ }_{2} F_{1}\left(1, \frac{1}{2}+\frac{\varepsilon}{2} ; 1-\frac{\varepsilon}{2} ;-p^{2} t^{2}\right)=\frac{1}{\left(1+t^{2} p^{2}\right)^{1 / 2+\frac{\varepsilon}{2}}}{ }_{2} F_{1}\left(\frac{1}{2}+\frac{\varepsilon}{2},-\frac{\varepsilon}{2} ; 1-\frac{\varepsilon}{2} ; q\right) .
$$

The last ${ }_{2} F_{1}$-function admits the following representation,

$$
\begin{aligned}
{ }_{2} F_{1}\left(\frac{1}{2}+\frac{\varepsilon}{2},-\frac{\varepsilon}{2} ; 1-\frac{\varepsilon}{2} ; q\right) & =\sum_{m=0}^{\infty} \frac{\Gamma\left(m+\frac{1}{2}+\frac{\varepsilon}{2}\right)}{m ! \Gamma\left(\frac{1}{2}+\frac{\varepsilon}{2}\right)} \frac{\left(-\frac{\varepsilon}{2}\right)}{m-\frac{\varepsilon}{2}} q^{m} \\
& =1-\frac{\varepsilon}{2} \sum_{m=1}^{\infty} \frac{\Gamma\left(m+\frac{1}{2}+\frac{\varepsilon}{2}\right)}{m ! \Gamma\left(\frac{1}{2}+\frac{\varepsilon}{2}\right)} \frac{1}{m-\frac{\varepsilon}{2}} q^{m}
\end{aligned}
$$

By using the integral representation $1 /\left(m-\frac{\varepsilon}{2}\right)=\int_{0}^{1} d z z^{m-1-\frac{\varepsilon}{2}}$, we find $\left(z_{1}=z q\right)$,

$$
\begin{aligned}
{ }_{2} F_{1}\left(\frac{1}{2}+\frac{\varepsilon}{2},-\frac{\varepsilon}{2} ; 1-\frac{\varepsilon}{2} ; q\right) & =1-\frac{\varepsilon}{2} \int_{0}^{1} \frac{d z}{z^{1+\frac{\varepsilon}{2}}}\left[\frac{1}{(1-z q)^{\frac{1}{2}+\frac{\varepsilon}{2}}}-1\right] \\
& =1-\frac{\varepsilon}{2} \int_{0}^{q} \frac{d z_{1} q^{\frac{\varepsilon}{2}}}{z_{1}^{1+\frac{\varepsilon}{2}}}\left[\frac{1}{\left(1-z_{1}\right)^{\frac{1}{2}+\frac{\varepsilon}{2}}}-1\right]
\end{aligned}
$$


so that eq. (A.8) reads,

$$
\sum_{m=0}^{\infty} \frac{\Gamma\left(m+\frac{3}{2}+\frac{\varepsilon}{2}\right)}{\Gamma\left(m+2-\frac{\varepsilon}{2}\right)}\left(-(t p)^{2}\right)^{m}=-\frac{\Gamma\left(\frac{1}{2}+\frac{\varepsilon}{2}\right)}{\Gamma\left(1-\frac{\varepsilon}{2}\right)(t p)^{2}}\left[\frac{1}{\left(1+t^{2} p^{2}\right)^{1 / 2+\frac{\varepsilon}{2}}}\left\{1-\frac{\varepsilon}{2} J_{1}^{(3)}(q)\right\}-1\right],
$$

where $J^{(3)}(q)$ is defined in eq. (3.7).

The final result for $F_{3}(t)$ is,

$$
F_{1}^{(3)}(t)=-\frac{(2-\varepsilon)(3+2 \varepsilon)}{4(1+\varepsilon) t^{2}} \hat{K}_{2} I_{1}^{(3)}(t), \quad I_{1}^{(3)}(t)=\int_{0}^{1} d p p^{\varepsilon}(1-p)^{-1 / 2-\varepsilon} f_{1}^{(3)}(p t),
$$

where

$$
f_{1}^{(3)}(p t)=\frac{1}{\left(1+t^{2} p^{2}\right)^{1 / 2+\frac{\varepsilon}{2}}}\left\{1-\frac{\varepsilon}{2} J^{(3)}(q)\right\}-1 .
$$

The third ${ }_{4} F_{3}$-hypergeometric functions of $J_{1,1,2}$ and $J_{1,1,1}, F_{2}^{(3)}(t)$ and $F_{3}^{(3)}(t)$, admit the following series representations,

$$
\begin{aligned}
F_{2}^{(3)}(t) & \equiv{ }_{4} F_{3}\left(\begin{array}{c}
1, \frac{1}{2}+\frac{\varepsilon}{2}, 1+\varepsilon, \frac{1}{2}+\varepsilon \\
2-\frac{\varepsilon}{2}, \frac{3}{4}+\frac{\varepsilon}{2}, \frac{5}{4}+\frac{\varepsilon}{2}
\end{array} \mid-t^{2}\right) \\
& =\sum_{m=0}^{\infty} \frac{\Gamma\left(m+\frac{1}{2}+\frac{\varepsilon}{2}\right) \Gamma\left(m+\frac{1}{2}+\varepsilon\right) \Gamma(m+1+\varepsilon)}{\Gamma\left(m+2-\frac{\varepsilon}{2}\right) \Gamma\left(m+\frac{3}{4}+\frac{\varepsilon}{2}\right) \Gamma\left(m+\frac{5}{4}+\frac{\varepsilon}{2}\right)} \frac{\Gamma\left(2-\frac{\varepsilon}{2}\right) \Gamma\left(\frac{3}{4}+\frac{\varepsilon}{2}\right) \Gamma\left(\frac{5}{4}+\frac{\varepsilon}{2}\right)}{\Gamma\left(\frac{1}{2}+\frac{\varepsilon}{2}\right) \Gamma(1+\varepsilon) \Gamma\left(\frac{1}{2}+\varepsilon\right)}\left(-t^{2}\right)^{m}, \\
F_{3}^{(3)}(t) & \equiv{ }_{4} F_{3}\left(\begin{array}{c}
1, \frac{1}{2}+\frac{\varepsilon}{2}, \varepsilon, \frac{1}{2}+\varepsilon \\
2-\frac{\varepsilon}{2}, \frac{3}{4}+\frac{\varepsilon}{2}, \frac{5}{4}+\frac{\varepsilon}{2}
\end{array} \mid-t^{2}\right) \\
& =\sum_{m=0}^{\infty} \frac{\Gamma\left(m+\frac{1}{2}+\frac{\varepsilon}{2}\right) \Gamma\left(m+\frac{1}{2}+\varepsilon\right) \Gamma(m+\varepsilon)}{\Gamma\left(m+2-\frac{\varepsilon}{2}\right) \Gamma\left(m+\frac{3}{4}+\frac{\varepsilon}{2}\right) \Gamma\left(m+\frac{5}{4}+\frac{\varepsilon}{2}\right)} \frac{\Gamma\left(2-\frac{\varepsilon}{2}\right) \Gamma\left(\frac{3}{4}+\frac{\varepsilon}{2}\right) \Gamma\left(\frac{5}{4}+\frac{\varepsilon}{2}\right)}{\Gamma\left(\frac{1}{2}+\frac{\varepsilon}{2}\right) \Gamma(\varepsilon) \Gamma\left(\frac{1}{2}+\varepsilon\right)}\left(-t^{2}\right)^{m} .
\end{aligned}
$$

Proceeding in a similar way we obtain,

$$
\begin{aligned}
& F_{2}^{(3)}(t)=\frac{(2-\varepsilon)(1+2 \varepsilon)}{2(1-\varepsilon) t^{2}} \hat{K}_{2} \int_{0}^{1} d p \frac{p^{2 \varepsilon-2}}{(1-p)^{1 / 2+\varepsilon}}\left[\left(1+t^{2} p^{2}\right)^{1 / 2-\frac{\varepsilon}{2}}\left\{1-\frac{\varepsilon}{2} J_{2}^{(3)}(q)\right\}-1\right], \\
& F_{3}^{(3)}(t)=\frac{2 \varepsilon(2-\varepsilon)(1+2 \varepsilon)}{(1-\varepsilon)(1-2 \varepsilon) t^{2}} \hat{K}_{2} \int_{0}^{1} d p \frac{p^{2 \varepsilon-3}}{(1-p)^{-1 / 2+\varepsilon}}\left[\left(1+t^{2} p^{2}\right)^{1 / 2-\frac{\varepsilon}{2}}\left\{1-\frac{\varepsilon}{2} J_{2}^{(3)}(q)\right\}-1\right],
\end{aligned}
$$

where

$$
J_{2}^{(3)}(q)=q^{\frac{\epsilon}{2}} \int_{0}^{q} d z\left((1-z)^{-\frac{\epsilon}{2}+\frac{1}{2}}-1\right) z^{-\frac{\epsilon}{2}-1}
$$

\section{B Relations between integrals $J_{2}^{(j)}(q)$ and $J^{(j)}(q)(j=1,3)$}

It is very convenient to present the results for $F_{j}^{(1)}(t)$ and $F_{j}^{(3)}(t)(j=2,3)$ shown in the eqs. (A.18) and (A.51), respectively, as integrals whose subintegral expressions depend on the universal structures $J^{(1)}(q)$ and $J^{(3)}(q)$. This is the subject of this appendix. In particular, this makes it possible to express the integrals contributing to $F_{j}^{(1)}(t)$ and $F_{j}^{(3)}(t)$ $(j=2,3)$, through the corresponding integrals appearing in $F_{1}^{(1)}(t)$ and $F_{1}^{(3)}(t)$ shown in eqs. (A.15) and (A.47) (see the evaluation in appendix C below), which strongly helps in building eMPL representations for $J_{1,1,2}(t)$ and $J_{1,1,1}(t)$. 
We first express integral $J_{2}^{(1)}(q)$ as,

$$
J_{2}^{(1)}(q)=\int_{0}^{q} \frac{d z q^{\varepsilon}}{z^{1+\varepsilon}}\left[\frac{1-z}{\sqrt{1-z}}-1\right]=J^{(1)}(q)-\bar{J}^{(1)}(q),
$$

where

$$
\bar{J}^{(1)}(q)=\int_{0}^{q} \frac{d z q^{\varepsilon}}{z^{\varepsilon}} \frac{1}{\sqrt{1-z}} .
$$

Secondly, by performing an integration by parts,

$$
J_{2}^{(1)}(q)=-\left.\frac{1}{\varepsilon} \frac{q^{\varepsilon}}{z^{\varepsilon}}[\sqrt{1-z}-1]\right|_{0} ^{q}+\frac{1}{\varepsilon} \int_{0}^{q} \frac{d z q^{\varepsilon}}{z^{\varepsilon}} \frac{(-1 / 2)}{\sqrt{1-z}}=\frac{1}{\varepsilon}[1-\sqrt{1-q}]-\frac{1}{2 \varepsilon} \bar{J}^{(1)}(q),
$$

and, thus, from (B.3),

$$
\bar{J}^{(1)}(q)=2[1-\sqrt{1-q}]-2 \varepsilon J_{2}^{(1)}(q) .
$$

By combining (B.1) and (B.4) we arrive at,

$$
(1-2 \varepsilon) J_{2}^{(1)}(q)=J^{(1)}(q)-2[1-\sqrt{1-q}] .
$$

Results for $F_{2}^{(1)}(t)$ and $F_{3}^{(1)}(t)$ can be rewritten as,

$$
\begin{aligned}
& F_{2}^{(1)}(t)=\frac{(1-\varepsilon)}{(1-2 \varepsilon) t^{2}} \hat{K}_{1} \int_{0}^{1} \frac{d p p^{\varepsilon-2}}{(1-p)^{1 / 2+\varepsilon}}\left[\left(1+t^{2} p^{2}\right)^{1 / 2}\left\{1-2 \varepsilon \sqrt{1-q}-\varepsilon J^{(1)}(q)\right\}-(1-2 \varepsilon)\right], \\
& F_{3}^{(1)}(t)=\frac{2 \varepsilon(1-\varepsilon)}{(1-2 \varepsilon)^{2} t^{2}} \hat{K}_{1} \int_{0}^{1} \frac{d p p^{\varepsilon-3}}{(1-p)^{\varepsilon-1 / 2}}\left[\left(1+t^{2} p^{2}\right)^{1 / 2}\left\{1-2 \varepsilon \sqrt{1-q}-\varepsilon J^{(1)}(q)\right\}-(1-2 \varepsilon)\right],
\end{aligned}
$$

where $J^{(1)}(q)$ is defined in eq. (3.7).

We can simplify these results by observing that,

$$
q=\frac{t^{2} p^{2}}{1+t^{2} p^{2}}, \quad 1-q=\frac{1}{1+t^{2} p^{2}}
$$

and, thus,

$$
\begin{gathered}
{\left[\left(1+t^{2} p^{2}\right)^{1 / 2}\left\{1-2 \varepsilon \sqrt{1-q}-\varepsilon J^{(1)}(q)\right\}-(1-2 \varepsilon)\right]} \\
=\left[\left(1+t^{2} p^{2}\right)^{1 / 2}\left\{1-\varepsilon J^{(1)}(q)\right\}-1\right] \equiv f_{2}^{(1)}(p t) .
\end{gathered}
$$

The final results for $F_{2}^{(1)}(t)$ and $F_{2}^{(1)}(t)$ read,

$$
\begin{array}{llrl}
F_{2}^{(1)}(t) & =\frac{(1-\varepsilon)}{\left({ }^{(} 1-2 \varepsilon\right) t^{2}} \hat{K}_{1} I_{2}^{(1)}(t), & I_{2}^{(1)}(t) & =\int_{0}^{1} d p p^{\varepsilon-2}(1-p)^{-1 / 2-\varepsilon} f_{2}^{(1)}(p t), \\
F_{3}^{(1)}(t) & =\frac{2 \varepsilon(1-\varepsilon)}{(1-2 \varepsilon)^{2} t^{2}} \hat{K}_{1} I_{3}^{(1)}(t), & I_{3}^{(1)}(t) & =\int_{0}^{1} d p p^{\varepsilon-3}(1-p)^{1 / 2-\varepsilon} f_{2}^{(1)}(p t) .
\end{array}
$$

For small $p$ values one can see from eq. (B.8) that,

$$
f_{2}^{(1)}(p t)=\frac{(1-2 \varepsilon)}{(1-\varepsilon)} \frac{(p t)^{2}}{2}+O\left(p^{4}\right),
$$


therefore integral $I_{3}^{(1)}(t)$ is singular. We define a new integral, $\tilde{I}_{3}^{(1)}(t)$, by extracting the leading asymptotic from $f_{2}^{(1)}(p t)$. We have,

$$
\tilde{f}_{2}^{(1)}(p t)=f_{2}^{(1)}(p t)-\frac{(1-2 \varepsilon)}{(1-\varepsilon)} \frac{(p t)^{2}}{2},
$$

and,

$$
I_{3}^{(1)}(t)=\tilde{I}_{3}^{(1)}(t)+\frac{(1-2 \varepsilon)^{2}}{2 \varepsilon(1-\varepsilon)} \frac{t^{2}}{\hat{K}_{1}}
$$

where,

$$
\tilde{I}_{3}^{(1)}(t)=\int_{0}^{1} d p p^{\varepsilon-3}(1-p)^{1 / 2-\varepsilon} \tilde{f}_{2}^{(1)}(p t)
$$

and,

$$
F_{3}^{(1)}(t)=1+\frac{2 \varepsilon(1-\varepsilon)}{(1-2 \varepsilon)^{2} t^{2}} \hat{K}_{1} \tilde{I}_{3}^{(1)}(t),
$$

We now represent integral $J_{2}^{(3)}(q)$ through $J^{(3)}(q)$. First, as for (B.1) we can represent integral $J_{2}^{(3)}(q)$ as,

$$
J_{2}^{(3)}(q)=J_{1}^{(3)}(q)-\bar{J}^{(3)}(q),
$$

where

$$
\bar{J}^{(3)}(q)=\int_{0}^{q} \frac{d z q^{\varepsilon / 2}}{z^{\varepsilon / 2}} \frac{1}{(1-z)^{(1-\varepsilon) / 2}} .
$$

We then perform an integration by parts for $J_{2}^{(3)}(q)$,

$$
\begin{aligned}
J_{2}^{(3)}(q) & \left.=-\frac{2}{\varepsilon} \frac{q^{\varepsilon / 2}}{z^{\varepsilon / 2}}\left[(1-z)^{(1-\varepsilon) / 2}-1\right]\right]_{0}^{q}+\frac{2}{\varepsilon} \int_{0}^{q} \frac{d z q^{\varepsilon / 2}}{z^{\varepsilon / 2}} \frac{(-1 / 2)(1-\varepsilon)}{(1-z)^{(1-\varepsilon) / 2}} \\
& =\frac{2}{\varepsilon}\left[1-(1-q)^{(1-\varepsilon) / 2}\right]-\frac{1-\varepsilon}{\varepsilon} \bar{J}^{(3)}(q),
\end{aligned}
$$

and, thus,

$$
\bar{J}^{(3)}(q)=\frac{1}{1-\varepsilon}\left(2\left[1-(1-q)^{(1-\varepsilon) / 2}\right]-\varepsilon J_{2}^{(3)}(q)\right) .
$$

By combining (B.15) and (B.18),

$$
J_{2}^{(3)}(q)=\frac{1-\varepsilon}{1-2 \varepsilon} J^{(3)}(q)-\frac{2}{1-2 \varepsilon}\left[1-(1-q)^{(1-\varepsilon) / 2}\right] .
$$

For $F_{2}^{(3)}(t)$ and $F_{3}^{(3)}(t)$ we have,

$$
\begin{aligned}
F_{2}^{(3)}(t)= & \frac{(2-\varepsilon)(1+2 \varepsilon)}{2(1-\varepsilon)(1-2 \varepsilon) t^{2}} \hat{K}_{2} \int_{0}^{1} d p \frac{p^{2 \varepsilon-2}}{(1-p)^{1 / 2+\varepsilon}}\left[\left(1+t^{2} p^{2}\right)^{1 / 2-\frac{\varepsilon}{2}}\right. \\
& \left.\times\left\{1-\varepsilon-\varepsilon(1-q)^{(1-\varepsilon) / 2}-\frac{\varepsilon}{2}(1-\varepsilon) J^{(3)}(y)\right\}-(1-2 \varepsilon)\right], \\
F_{3}^{(3)}(t)= & \frac{2 \varepsilon(2-\varepsilon)(1+2 \varepsilon)}{(1-\varepsilon)(1-2 \varepsilon)^{2} t^{2}} \hat{K}_{2} \int_{0}^{1} d p \frac{p^{2 \varepsilon-3}}{(1-p)^{-1 / 2+\varepsilon}}\left[\left(1+t^{2} p^{2}\right)^{1 / 2-\frac{\varepsilon}{2}}\right. \\
& \left.\times\left\{1-\varepsilon-\varepsilon(1-q)^{(1-\varepsilon) / 2}-\frac{\varepsilon}{2}(1-\varepsilon) J^{(3)}(q)\right\}-(1-2 \varepsilon)\right],
\end{aligned}
$$

where $J^{(3)}(q)$ is defined in eq. (3.7). 
Again, by using (B.7) we can simplify these results,

$$
\begin{gathered}
{\left[\left(1+t^{2} p^{2}\right)^{1 / 2-\frac{\varepsilon}{2}}\left\{1-\varepsilon-\varepsilon(1-q)^{(1-\varepsilon) / 2}-\frac{\varepsilon}{2}(1-\varepsilon) J_{1}^{(3)}(q)\right\}-(1-2 \varepsilon)\right]} \\
=(1-\varepsilon)\left[\left(1+t^{2} p^{2}\right)^{1 / 2-\frac{\varepsilon}{2}}\left\{1-\frac{\varepsilon}{2} J^{(3)}(q)\right\}-1\right] \equiv(1-\varepsilon) f_{2}^{(3)}(p t) .
\end{gathered}
$$

We obtain,

$$
\begin{aligned}
F_{2}^{(3)}(t) & =\frac{(2-\varepsilon)(1+2 \varepsilon)}{2(1-2 \varepsilon) t^{2}} \hat{K}_{2} I_{2}^{(3)}(t), & I_{2}^{(3)}(t) & =\int_{0}^{1} d p \frac{p^{2 \varepsilon-2}}{(1-p)^{1 / 2+\varepsilon}} f_{2}^{(3)}(p t), \\
F_{3}^{(3)}(t) & =\frac{2 \varepsilon(2-\varepsilon)(1+2 \varepsilon)}{(1-2 \varepsilon)^{2} t^{2}} \hat{K}_{2} I_{3}^{(3)}(t), & I_{3}^{(3)}(t) & =\int_{0}^{1} d p \frac{p^{2 \varepsilon-3}}{(1-p)^{-1 / 2+\varepsilon}} f_{2}^{(3)}(p t) .
\end{aligned}
$$

As for $F_{3}^{(1)}(t)$, at small $p$ values one can see that,

$$
f_{2}^{(3)}(p t)=\frac{(1-2 \varepsilon)}{(2-\varepsilon)}(p t)^{2}+O\left(p^{4}\right) .
$$

Therefore we define a finite integral $\tilde{I}_{3}^{(3)}(t)$ as,

$$
\tilde{f}_{2}^{(3)}(p t)=f_{2}^{(3)}(p t)-\frac{(1-2 \varepsilon)}{(2-\varepsilon)}(p t)^{2},
$$

and,

$$
I_{3}^{(3)}(t)=\tilde{I}_{3}^{(3)}(t)+\frac{(1-2 \varepsilon)^{2}}{2 \varepsilon(2-\varepsilon)(1+2 \varepsilon)} \frac{t^{2}}{\hat{K}_{2}}
$$

where,

$$
\tilde{I}_{3}^{(3)}(t)=\int_{0}^{1} d p p^{2 \varepsilon-3}(1-p)^{1 / 2-\varepsilon} \tilde{f}_{2}^{(3)}(p t)
$$

and,

$$
F_{3}^{(3)}(t)=1+\frac{2 \varepsilon(2-\varepsilon)(1+2 \varepsilon)}{(1-2 \varepsilon)^{2} t^{2}} \hat{K}_{2} \tilde{I}_{3}^{(3)}(t)
$$

\section{Improved representations}

After the variable change $p=1-x^{2}$, the results obtained for $I_{2}^{(i)}(t), \tilde{I}_{2}^{(2)}(t), \tilde{I}_{3}^{(2)}(t)$ and $\tilde{I}_{3}^{(i)}(t)(i=1,3)$, eqs. (B.9), (B.13), (A.33), (A.34), (B.22) and (B.26) respectively, contain integration kernels of the form $x^{a} / y(x), a>2$ and are therefore not optimal for expressing them in terms of eMPLs. Here we present alternative representations for the integrals $I_{2}^{(i)}(t), \tilde{I}_{2}^{(2)}(t), \tilde{I}_{3}^{(2)}(t)$ and $\tilde{I}_{3}^{(i)}(t)$, which can be expressed in terms of $I_{1}^{(i)}(t)$ and $I_{1}^{(2)}(t)$, shown in eqs. (A.15), (A.29) and (A.47), and simpler terms. These representations depend on kernels with at most simple poles and $x^{2} / y(x)$. 
Integrals $I_{2}^{(1)}(t)$ and $\tilde{I}_{3}^{(1)}(t)$. Using the following property,

$$
\frac{1}{p(1-p)}=\frac{1}{p} \frac{1}{1-p}
$$

we can split the result (B.9) for $I_{2}^{(1)}(t)$ as the combination of two parts:

$$
I_{2}^{(1)}(t)=\int_{0}^{1} \frac{d p}{p^{1-\varepsilon}(1-p)^{1 / 2+\varepsilon}} f_{2}^{(1)}(p t)+\int_{0}^{1} \frac{d p(1-p)^{1 / 2-\varepsilon}}{p^{2-\varepsilon}} f_{2}^{(1)}(p t)=\hat{I}_{21}^{(1)}(t)+\hat{I}_{22}^{(1)}(t)
$$

where $f_{2}^{(1)}(p t)$ is defined in eq. (B.8).

We note that,

$$
f_{2}^{(1)}(p t)=f_{1}^{(1)}(p t)+\frac{t^{2} p^{2}}{\left(1+t^{2} p^{2}\right)^{1 / 2}}\left\{1-\varepsilon J^{(1)}(q)\right\}
$$

where $f_{1}^{(1)}(p t)$ is defined in eq. (A.14).

Then,

$$
\begin{aligned}
\hat{I}_{21}^{(1)}(t) & =I_{1}^{(1)}(t)+t^{2} \int_{0}^{1} \frac{d p p^{1+\varepsilon}(1-p)^{1 / 2-\varepsilon}}{\left(1+t^{2} p^{2}\right)^{1 / 2}}\left\{1-\varepsilon J^{(1)}(q)\right\}=I_{1}^{(1)}(t)+t^{2} I_{21}^{(1)}(t), \\
\hat{I}_{22}^{(1)}(t) & =\int_{0}^{1} \frac{d p(1-p)^{1 / 2-\varepsilon}}{p^{2-\varepsilon}} f_{1}^{(1)}(p)+t^{2} \int_{0}^{1} \frac{d p p^{\varepsilon}(1-p)^{1 / 2-\varepsilon}}{\left(1+t^{2} p^{2}\right)^{1 / 2}}\left\{1-\varepsilon J^{(1)}(q)\right\} \\
& =\int_{0}^{1} \frac{d p(1-p)^{1 / 2-\varepsilon}}{p^{2-\varepsilon}} f_{1}^{(1)}(p)+t^{2} I_{22}^{(1)}(t) .
\end{aligned}
$$

We now integrate $\hat{I}_{22}^{(1)}(t)$ by parts as,

$$
\int_{0}^{1} \frac{d p}{p^{\alpha+1}} F(p)=-\frac{1}{\alpha}\left[\left.\frac{F(p)}{p^{\alpha}}\right|_{0} ^{1}-\int_{0}^{1} \frac{d p}{p^{\alpha}} \frac{d F(p)}{d p}\right]
$$

where $F(p)$ is some function and $\alpha=1-\varepsilon$. We have,

$$
\begin{aligned}
\hat{I}_{22}^{(1)}(t)=-\frac{1}{1-\varepsilon}[ & \left.\frac{(1-p)^{1 / 2-\varepsilon}}{p^{1-\varepsilon}} f_{2}^{(1)}(p)\right|_{0} ^{1}+\left(\frac{1}{2}-\varepsilon\right) \hat{I}_{21}^{(1)}(t) \\
& \left.-t^{2} I_{21}^{(1)}(t)+\varepsilon \int_{0}^{1} \frac{d p(1-p)^{1 / 2-\varepsilon}}{p^{1-\varepsilon}\left(1+t^{2} p^{2}\right)^{-1 / 2}} \frac{d J^{(1)}(q)}{d p}\right],
\end{aligned}
$$

where,

$$
\left.\frac{(1-p)^{1 / 2-\varepsilon}}{p^{1-\varepsilon}} f_{2}^{(1)}(p)\right|_{0} ^{1}=0
$$

and,

$$
\frac{d J^{(1)}(q)}{d p}=\frac{2}{p\left(1+p^{2} t^{2}\right)}\left[\varepsilon J^{(1)}(q)+\sqrt{1+p^{2} t^{2}}-1\right]=-\frac{2}{p\left(1+p^{2} t^{2}\right)^{1 / 2}} f_{1}^{(1)}(p) .
$$


The last term in (C.7) can be expressed as,

$$
\begin{aligned}
\varepsilon \int_{0}^{1} \frac{d p(1-p)^{1 / 2-\varepsilon}}{p^{1-\varepsilon}\left(1+t^{2} p^{2}\right)^{-1 / 2}} \frac{d J^{(1)}(q)}{d p} & =-2 \varepsilon \int_{0}^{1} \frac{d p(1-p)^{1 / 2-\varepsilon}}{p^{2-\varepsilon}} f_{1}^{(1)}(p) \\
& =-2 \varepsilon\left[\hat{I}_{22}^{(1)}(t)-t^{2} I_{22}^{(1)}(t)\right]
\end{aligned}
$$

and, by using (C.5), the r.h.s. of (C.7) takes the following form,

$$
-\frac{1}{1-\varepsilon}\left[\left(\frac{1}{2}-\varepsilon\right) \hat{I}_{21}^{(1)}(t)-(1-2 \varepsilon) t^{2} I_{22}^{(1)}(t)-2 \varepsilon \hat{I}_{22}^{(1)}(t)\right],
$$

which leads to,

$$
\hat{I}_{22}^{(1)}(t)=\frac{1-2 \varepsilon}{2(1-3 \varepsilon)}\left[-\hat{I}_{21}^{(1)}(t)+2 t^{2} I_{22}^{(1)}(t)\right] .
$$

Therefore, integral $I_{2}^{(1)}(t)$ can be represented as,

$$
I_{2}^{(1)}(t)=\frac{1}{2(1-3 \varepsilon)}\left[(1-4 \varepsilon) \hat{I}_{21}^{(1)}(t)+2(1-2 \varepsilon) t^{2} I_{22}^{(1)}(t)\right],
$$

and, by using (C.3), we can rewrite (C.13) in the following form,

$$
I_{2}^{(1)}(t)=\frac{1}{2(1-3 \varepsilon)}\left[(1-4 \varepsilon)\left(I_{1}^{(1)}(t)+t^{2} I_{21}^{(1)}(t)\right)+2(1-2 \varepsilon) t^{2} I_{22}^{(1)}(t)\right] .
$$

After the replacement $p=1-x^{2}$ (see eq. (4.25)), we have,

$$
\begin{aligned}
& I_{21}^{(1)}(t) \sim \int_{0}^{1} \frac{d p p^{\varepsilon}(1-p)^{1 / 2-\varepsilon}}{\left(1+t^{2} p^{2}\right)^{1 / 2}}=2 \int_{0}^{1} \frac{d x x^{2}}{t y}\left[\frac{1-x^{2}}{x^{2}}\right]^{\varepsilon}, \\
& I_{22}^{(1)}(t) \sim \int_{0}^{1} \frac{d p p^{1+\varepsilon}(1-p)^{-1 / 2-\varepsilon}}{\left(1+t^{2} p^{2}\right)^{1 / 2}}=2 \int_{0}^{1} \frac{d x\left(1-x^{2}\right)}{t y}\left[\frac{1-x^{2}}{x^{2}}\right]^{\varepsilon} .
\end{aligned}
$$

We now consider integral $\tilde{I}_{3}^{(1)}(t)$, eq. (B.13). Repeating the derivation for $I_{2}^{(1)}(t)$, we obtain,

$$
\tilde{I}_{3}^{(1)}(t)=\frac{1-2 \varepsilon}{2(2-3 \varepsilon)}\left[2 t^{2}\left(I_{1}^{(1)}(t)-I_{31}^{(1)}(t)\right)-I_{2}^{(1)}(t)+\frac{(5-6 \varepsilon)}{(1-\varepsilon)} \frac{t^{2}}{\hat{K}_{1}}\right]
$$

where,

$$
I_{31}^{(1)}(t)=\int_{0}^{1} \frac{d p p^{\varepsilon}(1-p)^{-1 / 2-\varepsilon}}{\left(1+t^{2} p^{2}\right)^{1 / 2}}\left\{1-\varepsilon J_{1}^{(1)}(q)\right\}
$$

with,

$$
\int_{0}^{1} \frac{d p p^{\varepsilon}}{(1-p)^{1 / 2+\varepsilon}\left(1+t^{2} p^{2}\right)^{1 / 2}}=2 \int_{0}^{1} \frac{d x}{t y}\left[\frac{1-x^{2}}{x^{2}}\right]^{\varepsilon} .
$$

Since $I_{22}^{(1)}(t)=I_{31}^{(1)}(t)-I_{21}^{(1)}(t)$, eq. (C.14) can be replaced by (4.51) with $i=1$ in the main text. 
Integrals $\boldsymbol{I}_{\mathbf{2}}^{(\mathbf{3})}(\boldsymbol{t})$ and $\tilde{\boldsymbol{I}}_{\mathbf{3}}^{(\mathbf{3})}(\boldsymbol{t})$. Using (C.1), we can split the result (B.9) for $I_{2}^{(3)}(t)$ as:

$$
I_{2}^{(3)}(t)=\int_{0}^{1} \frac{d p}{p^{1-2 \varepsilon}(1-p)^{1 / 2+\varepsilon}} f_{2}^{(3)}(p)+\int_{0}^{1} \frac{d p(1-p)^{1 / 2-\varepsilon}}{p^{2-2 \varepsilon}} f_{2}^{(3)}(p)=\hat{I}_{21}^{(3)}(t)+\hat{I}_{22}^{(3)}(t),
$$

where $f_{2}^{(3)}(t p)$ is defined in eq. (B.21).

We note that,

$$
f_{2}^{(3)}(p)=f_{1}^{(3)}(p)+\frac{t^{2} p^{2}}{\left(1+t^{2} p^{2}\right)^{(1+\varepsilon) / 2}}\left\{1-\frac{\varepsilon}{2} J^{(3)}(q)\right\}
$$

where $f_{1}^{(3)}(p t)$ is defined in eq. (A.48).

Then,

$$
\begin{aligned}
\hat{I}_{21}^{(3)}(t) & =I_{1}^{(3)}(t)+t^{2} \int_{0}^{1} \frac{d p p^{2 \varepsilon}(1-p)^{1 / 2-\varepsilon}}{\left(1+t^{2} p^{2}\right)^{(1+\varepsilon) / 2}}\left\{1-\frac{\varepsilon}{2} J^{(3)}(q)\right\}=I_{1}^{(3)}(t)+t^{2} I_{21}^{(3)}(t) \\
\hat{I}_{22}^{(3)}(t) & =\int_{0}^{1} \frac{d p(1-p)^{1 / 2-\varepsilon}}{p^{2-2 \varepsilon}} f_{1}^{(3)}(p)+t^{2} \int_{0}^{1} \frac{d p p^{2 \varepsilon}(1-p)^{1 / 2-\varepsilon}}{\left(1+t^{2} p^{2}\right)^{(1+\varepsilon) / 2}}\left\{1-\frac{\varepsilon}{2} J^{(3)}(q)\right\} \\
& =\int_{0}^{1} \frac{d p(1-p)^{1 / 2-\varepsilon}}{p^{2-2 \varepsilon}} f_{1}^{(3)}(p)+t^{2} I_{22}^{(3)}(t)
\end{aligned}
$$

Now we integrate integral $\hat{I}_{22}^{(3)}(t)$ by parts as in eq. (C.6) with $\alpha=1-2 \varepsilon$. We have,

$$
\begin{aligned}
\hat{I}_{22}^{(3)}(t)=-\frac{1}{1-2 \varepsilon}[ & {\left[\left.\frac{(1-p)^{1 / 2-\varepsilon}}{p^{1-2 \varepsilon}} f_{2}^{(3)}(p)\right|_{0} ^{1}+\left(\frac{1}{2}-\varepsilon\right) \hat{I}_{21}^{(3)}(t)\right.} \\
& \left.-(1-\varepsilon) t^{2} I_{22}^{(3)}(t)+\frac{\varepsilon}{2} \int_{0}^{1} \frac{d p(1-p)^{1 / 2-\varepsilon}}{p^{1-2 \varepsilon}\left(1+t^{2} p^{2}\right)^{-(1-\varepsilon) / 2}} \frac{d J^{(3)}(q)}{d p}\right],
\end{aligned}
$$

with,

$$
\left.\frac{(1-p)^{1 / 2-\varepsilon}}{p^{1-2 \varepsilon}} f_{2}^{(3)}(p)\right|_{0} ^{1}=0
$$

and,

$$
\frac{d J^{(3)}(q)}{d p}=-\frac{2}{p\left(1+p^{2} t^{2}\right)^{(1-\varepsilon) / 2}} f_{1}^{(3)}(p)
$$

Therefore, the last term in (C.24) can be represented as,

$$
\begin{aligned}
\frac{\varepsilon}{2} \int_{0}^{1} \frac{d p(1-p)^{1 / 2-\varepsilon}}{p^{1-2 \varepsilon}\left(1+t^{2} p^{2}\right)^{-(1-\varepsilon) / 2}} \frac{d J^{(3)}(q)}{d p} & =-\varepsilon \int_{0}^{1} \frac{d p(1-p)^{1 / 2-\varepsilon}}{p^{2-2 \varepsilon}} f_{1}^{(3)}(p) \\
& =-\varepsilon\left[\hat{I}_{22}^{(3)}(t)-t^{2} I_{22}^{(3)}(t)\right]
\end{aligned}
$$

and, using (C.23), the r.h.s. of (C.24) takes the following form:

$$
-\frac{1}{1-2 \varepsilon}\left[\left(\frac{1}{2}-\varepsilon\right) \hat{I}_{21}^{(3)}(t)-(1-2 \varepsilon) t^{2} I_{22}^{(3)}(t)-\varepsilon \hat{I}_{22}^{(3)}(t)\right],
$$


which leads to,

$$
\hat{I}_{22}^{(3)}(t)=\frac{1-2 \varepsilon}{2(1-3 \varepsilon)}\left[-\hat{I}_{21}^{(3)}(t)+2 t^{2} I_{22}^{(3)}(t)\right] .
$$

Therefore integral $I_{2}^{(3)}(t)$ can be represented as,

$$
I_{2}^{(3)}(t)=\frac{1}{2(1-3 \varepsilon)}\left[(1-4 \varepsilon) \hat{I}_{21}^{(3)}(t)+2(1-2 \varepsilon) t^{2} I_{22}^{(3)}(t)\right],
$$

and, by using (C.21), we have,

$$
I_{2}^{(3)}(t)=\frac{1}{2(1-3 \varepsilon)}\left[(1-4 \varepsilon)\left(I_{1}^{(3)}(t)+t^{2} I_{21}^{(3)}(t)\right)+2(1-2 \varepsilon) t^{2} I_{22}^{(3)}(t)\right] .
$$

After the replacement $p=1-x^{2}$, we have,

$$
\begin{aligned}
& I_{21}^{(3)}(t) \sim \int_{0}^{1} \frac{d p p^{2 \varepsilon}(1-p)^{1 / 2-\varepsilon}}{\left(1+t^{2} p^{2}\right)^{(1+\varepsilon) / 2}}=2 \int_{0}^{1} \frac{d x x^{2}}{t \hat{y}}\left[\frac{\left(1-x^{2}\right)^{2}}{x^{2}(t y)}\right]^{\varepsilon}, \\
& I_{22}^{(3)}(t) \sim \int_{0}^{1} \frac{d p p^{1+2 \varepsilon}(1-p)^{-1 / 2-\varepsilon}}{\left(1+t^{2} p^{2}\right)^{(1+\varepsilon) / 2}}=2 \int_{0}^{1} \frac{d x\left(1-x^{2}\right)}{t y}\left[\frac{\left(1-x^{2}\right)^{2}}{x^{2}(t y)}\right]^{\varepsilon} .
\end{aligned}
$$

Now we consider integral $\tilde{I}_{3}^{(3)}(t)$, eq. (B.26). Proceeding as above we have,

$$
\tilde{I}_{3}^{(3)}(t)=\frac{1-2 \varepsilon}{2(2-3 \varepsilon)}\left[2 t^{2}\left(I_{1}^{(3)}(t)-I_{31}^{(3)}(t)\right)-I_{2}^{(3)}(t)\right],
$$

where,

$$
I_{31}^{(3)}(t)=\int_{0}^{1} \frac{d p p^{2 \varepsilon}(1-p)^{-1 / 2-\varepsilon}}{\left(1+t^{2} p^{2}\right)^{(1+\varepsilon) / 2}}\left\{1-\frac{\varepsilon}{2} J_{1}^{(3)}(q)\right\}
$$

with,

$$
\int_{0}^{1} \frac{d p p^{2 \varepsilon}}{(1-p)^{1 / 2+\varepsilon}\left(1+t^{2} p^{2}\right)^{(1+\varepsilon) / 2}}=2 \int_{0}^{1} \frac{d x}{t y}\left[\frac{\left(1-x^{2}\right)^{2}}{x^{2}(t y)}\right]^{\varepsilon} .
$$

Since $I_{22}^{(3)}(t)=I_{31}^{(3)}(t)-I_{21}^{(3)}(t)$, eq. (C.31) can be replaced by (4.51) with $i=3$ in the main text.

Integrals $\tilde{\boldsymbol{I}}_{\mathbf{2}}^{(\mathbf{2})}(\boldsymbol{t})$ and $\tilde{\boldsymbol{I}}_{\mathbf{3}}^{(\mathbf{2})}(\boldsymbol{t})$. Using (C.1), we can split the result (A.33) for $\tilde{I}_{2}^{(2)}(t)$ as the combination of two parts:

$$
\tilde{I}_{2}^{(2)}(t)=\int_{0}^{1} \frac{d p}{p^{1-3 \varepsilon}(1-p)^{1 / 2+\varepsilon}} \tilde{f}_{2}^{(2)}(t p)+\int_{0}^{1} \frac{d p(1-p)^{1 / 2-\varepsilon}}{p^{2-2 \varepsilon}} \tilde{f}_{2}^{(2)}(t p)=\tilde{I}_{21}^{(2)}(t)+\tilde{I}_{22}^{(2)}(t) .
$$

It is convenient to express $\tilde{I}_{21}^{(2)}(t)$ as,

$$
\tilde{I}_{21}^{(2)}(t)=\hat{I}_{21}^{(2)}(t)-\frac{2 t^{1-\varepsilon}}{(1-\varepsilon)(1+2 \varepsilon)} \frac{1}{\hat{K}_{2}}, \quad \hat{I}_{21}^{(2)}(t)=I_{1}^{(2)}(t)+t^{2} I_{21}^{(2)}(t),
$$


where $f_{2}^{(2)}=\left(1+t^{2} p^{2}\right) f_{1}^{(2)}$ and,

$$
I_{21}^{(2)}(t)=\int_{0}^{1} \frac{d p p^{1+3 \varepsilon}}{(1-p)^{1 / 2+\varepsilon}\left(1+t^{2} p^{2}\right)^{1 / 2+\varepsilon}} J^{(2)}(t p),
$$

with,

$$
\int_{0}^{1} \frac{d p p^{3 \varepsilon+1}}{(1-p)^{1 / 2-\varepsilon}\left(1+t^{2} p^{2}\right)^{1 / 2+\varepsilon}}=2 \int_{0}^{1} \frac{d x\left(1-x^{2}\right)}{t y}\left[\frac{\left(1-x^{2}\right)^{3}}{x^{2}(t y)^{2}}\right]^{\varepsilon} .
$$

We now consider the second part of the r.h.s. of (C.37). We integrate the term $\tilde{I}_{22}^{(2)}(t)$ by parts as in eq. (C.6) with $\alpha=1-3 \varepsilon$. We have,

$$
\begin{aligned}
\tilde{I}_{22}^{(2)}(t)=\frac{1}{1-3 \varepsilon}[ & -\left.\frac{(1-p)^{1 / 2-\varepsilon}}{p^{1-3 \varepsilon}} \tilde{f}_{2}^{(2)}(p t)\right|_{0} ^{1}-(1 / 2-\varepsilon) \tilde{I}_{21}^{(2)}(t) \\
& +(1-2 \varepsilon) t^{2} \int_{0}^{1} \frac{d p p^{3 \varepsilon}(1-p)^{1 / 2-\varepsilon}}{\left(1+t^{2} p^{2}\right)^{1 / 2+\varepsilon}} J^{(2)}(t p) \\
& \left.+\int_{0}^{1} \frac{d p(1-p)^{1 / 2-\varepsilon}}{p^{1-3 \varepsilon}\left(1+t^{2} p^{2}\right)^{-(1-\varepsilon) / 2}} \frac{d J^{(2)}(t p)}{d p}-\int_{0}^{1} \frac{d p(1-p)^{1 / 2-\varepsilon}}{p^{1-3 \varepsilon}} \frac{t^{1-\varepsilon}}{p^{\varepsilon}}\right],
\end{aligned}
$$

with,

$$
\left.\frac{(1-p)^{1 / 2-\varepsilon}}{p^{1-3 \varepsilon}} \tilde{f}^{(2)}(p)\right|_{0} ^{1}=0
$$

and,

$$
\frac{d J^{(2)}(t p)}{d p}=\frac{t^{1-\varepsilon}}{p^{\varepsilon}\left(1+p^{2} t^{2}\right)^{1 / 2-\varepsilon}},
$$

and the last terms in the r.h.s. of (C.41) cancel exactly. Eq. (C.41) can be represented as,

$$
\frac{1}{1-3 \varepsilon}\left[-(1 / 2-\varepsilon) \tilde{I}_{21}^{(2)}(t)+(1-2 \varepsilon) t^{2} I_{22}^{(2)}(t)\right]
$$

where,

$$
I_{22}^{(2)}(t)=\int_{0}^{1} \frac{d p p^{3 \varepsilon}(1-p)^{1 / 2-\varepsilon}}{\left(1+t^{2} p^{2}\right)^{1 / 2+\varepsilon}} J^{(2)}(t p)
$$

is such that,

$$
\int_{0}^{1} \frac{d p p^{3 \varepsilon}(1-p)^{1 / 2-\varepsilon}}{\left(1+t^{2} p^{2}\right)^{1 / 2+\varepsilon}}=2 \int_{0}^{1} \frac{d x x^{2}}{t y}\left[\frac{\left(1-x^{2}\right)^{3}}{x^{2}(t y)^{2}}\right]^{\varepsilon} .
$$

Therefore we have that,

$$
\begin{aligned}
\tilde{I}_{2}^{(2)}(t) & =\frac{1}{2(1-3 \varepsilon)}\left[(1-4 \varepsilon) \tilde{I}_{21}^{(2)}(t)+2(1-2 \varepsilon) t^{2} I_{22}^{(2)}(t)\right] \\
& =\frac{1}{2(1-3 \varepsilon)}\left[(1-4 \varepsilon)\left(I_{1}^{(2)}(t)+t^{2} I_{21}^{(2)}(t)-\frac{2 t^{1-\varepsilon}}{(1-\varepsilon)(1+2 \varepsilon)} \frac{1}{\hat{K}_{2}}\right)+2(1-2 \varepsilon) t^{2} I_{22}^{(2)}(t)\right] .
\end{aligned}
$$


Now we consider the integral $\tilde{I}_{3}^{(2)}(t)$ shown in (A.34). Proceeding as above we have for $\tilde{I}_{3}^{(2)}(t)$,

$$
\tilde{I}_{3}^{(2)}(t)=\frac{1-2 \varepsilon}{2(2-3 \varepsilon)}\left[2 t^{2}\left(I_{1}^{(2)}(t)-I_{31}^{(2)}(t)\right)-\tilde{I}_{3}^{(2)}(t)\right]
$$

where,

$$
I_{31}^{(2)}(t)=\int_{\delta}^{1} \frac{d p p^{3 \varepsilon}(1-p)^{-1 / 2-\varepsilon}}{\left(1+t^{2} p^{2}\right)^{1 / 2+\varepsilon}} J^{(2)}(p)
$$

with,

$$
I_{31}^{(2)}(t) \sim \int_{0}^{1} \frac{d p p^{3 \varepsilon}(1-p)^{-1 / 2-\varepsilon}}{\left(1+t^{2} p^{2}\right)^{1 / 2+\varepsilon}}=2 \int_{0}^{1} \frac{d x}{t y}\left[\frac{\left(1-x^{2}\right)^{3}}{x^{2}(t y)^{2}}\right]^{\varepsilon} .
$$

Since $I_{22}^{(2)}(t)=I_{31}^{(2)}(t)-I_{21}^{(2)}(t)$, eq. (C.47) can be replaced by (4.58) in the main text of the paper.

\section{Leading terms of the $\varepsilon$-expansion and one-fold integrals}

In this section we derive a one-fold integral representation for the first two orders of the $\epsilon$-expansion of $J_{1,2,2}, J_{1,1,2}$ and $J_{1,1,1}$, (see eqs. (2.2)-(2.4), respectively). These representations generalise the results of refs. [6] and [56], where only the finite part of the expansion was considered. The first two $\epsilon$ orders considered here can be expressed as one-fold integrals over logarithms and dilogarithms with algebraic prefactors. A similar analysis shows that to arbitrary order of the dimensional regulator the result is in terms of one-fold integrals over higher weight MPLs.

Inner integrals. We start by considering integral $J^{(2)}(p)$, defined in eq. (3.7), at order $\epsilon^{0}$,

$$
J^{(2)}(p t, \varepsilon=0)=\int_{0}^{t p} \frac{d s}{\sqrt{1+s^{2}}},
$$

which can be evaluated directly by means of the variable change,

$$
s_{2}=\frac{\sqrt{1+s^{2}}-s}{\sqrt{1+s^{2}}+s}
$$

leading to,

$$
J^{(2)}(p t, \varepsilon=0)=\frac{1}{2} \int_{R_{2}}^{1} \frac{d s_{2}}{s_{2}}=-\frac{1}{2} \log R_{2} \equiv J_{0}^{(2)}(p),
$$

with

$$
R_{2}=\frac{\sqrt{1+t^{2} p^{2}}-t p}{\sqrt{1+t^{2} p^{2}}+t p}=\frac{1-\sqrt{q}}{1+\sqrt{q}} .
$$

By means of the same variable change, we evaluate the next $\epsilon$ order,

$$
J^{(2)}(p t)=\frac{1}{2^{1+\varepsilon}} \int_{R_{2}}^{1} \frac{d s_{2}}{s_{2}^{1+\varepsilon / 2}} \frac{\left(1+s_{2}\right)^{2 \varepsilon}}{\left(1-s_{2}\right)^{2 \varepsilon}}=J_{0}^{(2)}(p t)+\varepsilon J_{1}^{(2)}(p t)+\mathcal{O}\left(\epsilon^{2}\right),
$$


where $J_{0}^{(2)}(p t)$ is given in (D.3) and,

$$
J_{1}^{(2)}(p)=\frac{1}{8} \log ^{2} R_{2}+\zeta_{2}+\operatorname{Li}_{2}\left(-R_{2}\right)-\frac{1}{2} \operatorname{Li}_{2}\left(R_{2}\right) .
$$

We now consider integrals $J^{(1)}(p)$ and $J^{(3)}(p)$, defined in eq. (3.7), at order $\epsilon^{0}$,

$$
J^{(1)}(p, \varepsilon=0)=J^{(3)}(p, \varepsilon=0)=\int_{0}^{q} \frac{d z}{z}\left(\frac{1}{\sqrt{1-z}}-1\right) .
$$

By introducing a regulator $\delta$ we have,

$$
\int_{\delta}^{y} \frac{d z}{z}=\log q-\log \delta
$$

while the remaining term can be evaluated by the variable change,

$$
z=1-s^{2}, \quad s=\frac{\left(1-s_{1}\right)}{\left(1+s_{1}\right)},
$$

and,

$$
\int_{\delta}^{q} \frac{d z}{z \sqrt{1-z}}=\int_{\delta / 4}^{R_{1}} \frac{d s_{1}}{s_{1}}=\log R_{1}-\log \frac{\delta}{4}, \quad R_{1}=\frac{1-\sqrt{1-q}}{1+\sqrt{1-q}} .
$$

The full result can be written as,

$$
J^{(i)}(p, \varepsilon=0)=\log \left(4 R_{1}\right)-\log \delta=\log \frac{4 R_{1}}{q} \equiv J_{0}^{(i)}(p), \quad(i=1,3) .
$$

At the next order we have,

$$
J^{(i)}(p)=J_{0}^{(i)}(p)+\varepsilon J_{1}^{(i)}(p)+\mathcal{O}\left(\epsilon^{2}\right), \quad(i=1,3),
$$

where $J_{0}^{(1)}(p)=J_{0}^{(3)}(p)$ are given in (D.11) and,

$$
J_{1}^{(1)}(p)=\bar{J}_{1}^{(1)}(p)-2 \operatorname{Li}_{2}\left(-R_{1}\right), \quad J_{1}^{(3)}(p)=\frac{1}{2} \bar{J}_{1}^{(1)}(p)+2 \operatorname{Li}_{2}\left(R_{1}\right)-4 \operatorname{Li}_{2}\left(-R_{1}\right),
$$

with,

$$
\bar{J}_{1}^{(1)}(p)=\log q \log \left(4 R_{1}\right)-\frac{1}{2} \log ^{2} q-\log 4 \log R_{1} .
$$

Results for the sunrise integrals. Consider eqs. (4.42) and (4.47)-(4.50). In order to obtain the $\varepsilon$-expansion of $J_{1,2,2}$ up to and including $\mathcal{O}(\epsilon)$, we use the expressions for integrals $I^{(i)}(t)(i=1,3)$ in eq. (3.5) and their integral representations in eq. (3.6). We have,

$$
\begin{aligned}
I_{1,1}^{(1)}(t) & =\int_{0}^{1} \frac{d p}{p \sqrt{1-p}}\left(\frac{1}{\sqrt{1+p^{2} t^{2}}}-1\right)\left[1+\varepsilon l_{1}+\frac{\varepsilon^{2}}{2} l_{1}^{2}\right]+\mathcal{O}\left(\epsilon^{2}\right), \\
I_{1,2}^{(1)}(t) & =\int_{0}^{1} \frac{d p}{p \sqrt{1-p}} \frac{1}{\sqrt{1+p^{2} t^{2}}}\left[J_{0}^{(1)}+\varepsilon\left(l_{1} J_{0}^{(1)}+J_{1}^{(1)}\right)\right]+\mathcal{O}\left(\epsilon^{2}\right), \\
\left(\frac{t^{2}}{2}\right)^{\varepsilon} I_{1}^{(2)}(t) & =\int_{0}^{1} \frac{d p}{p \sqrt{1-p}} \frac{1}{\sqrt{1+p^{2} t^{2}}}\left[J_{0}^{(2)}+\varepsilon\left(l_{2} J_{0}^{(2)}+J_{1}^{(2)}\right)\right]+\mathcal{O}\left(\epsilon^{2}\right), \\
\left(\frac{t}{2}\right)^{\varepsilon} I_{1,1}^{(3)}(t) & =\int_{0}^{1} \frac{d p}{p \sqrt{1-p}}\left(\frac{1}{\sqrt{1+p^{2} t^{2}}}\left[1+\varepsilon l_{32}+\frac{\varepsilon^{2}}{2} l_{32}^{2}\right]-\left[1+\varepsilon l_{31}+\frac{\varepsilon^{2}}{2} l_{31}^{2}\right]\right)+\mathcal{O}\left(\epsilon^{2}\right), \\
\left(\frac{t}{2}\right)^{\varepsilon} I_{1,2}^{(3)}(t) & =\int_{0}^{1} \frac{d p}{p \sqrt{1-p}} \frac{1}{\sqrt{1+p^{2} t^{2}}}\left[J_{0}^{(3)}+\varepsilon\left(l_{32} J_{0}^{(3)}+J_{1}^{(3)}\right)\right]+\mathcal{O}\left(\epsilon^{2}\right),
\end{aligned}
$$


where,

$$
\begin{array}{rlrl}
l_{1}=\log \left(\frac{p}{1-p}\right), & l_{2}=\log \left(\frac{p^{3} t^{2}}{2(1-p)\left(1+p^{2} t^{2}\right)}\right) & =\log \left(\frac{p q}{2(1-p)}\right), \\
l_{31}=\log \left(\frac{p^{2} t}{2(1-p)}\right), & l_{32}=\log \left(\frac{p^{2} t}{2(1-p) \sqrt{1+p^{2} t^{2}}}\right)=\log \left(\frac{p \sqrt{q}}{2(1-p)}\right),
\end{array}
$$

with $J_{0}^{(i)}$ and $J_{1}^{(i)}(i=1,2,3)$ given in eqs. (D.5), (D.6), (D.11)-(D.14).

Combining all terms, we obtain the following finite expression,

$$
\begin{aligned}
& \hat{J}_{1,2,2}= \int_{0}^{1} \frac{d p}{p \sqrt{1-p}}\left[b_{0}+\frac{1}{\sqrt{1+p^{2} t^{2}}} B_{0}+\varepsilon\left(b_{1}+\frac{1}{\sqrt{1+p^{2} t^{2}}} B_{1}\right)\right]+\mathcal{O}\left(\epsilon^{2}\right), \\
& \hat{J}_{1,1,2}^{\mathrm{reg}}=\int_{0}^{1} \frac{d p}{p^{2} \sqrt{1-p}}\left[b_{0}-(p t)+\frac{1}{\sqrt{1+p^{2} t^{2}}} B_{0}+\varepsilon\left(b_{1}-(p t)\left(1+l_{31}\right)\right.\right. \\
&\left.\left.\quad+\frac{1}{\sqrt{1+p^{2} t^{2}}} B_{1}\right)\right]+\mathcal{O}\left(\epsilon^{2}\right), \\
& \hat{J}_{1,1,1}^{\mathrm{reg}}=\int_{0}^{1} \frac{d p \sqrt{1-p}}{p^{3}}\left[b_{0}-(p t)+\frac{(p t)^{2}}{4}\left(2 b_{0}-1\right)+\frac{1}{\sqrt{1+p^{2} t^{2}}} B_{0}+\varepsilon\left(b_{1}-(p t)\left(1+l_{31}\right)\right.\right. \\
&\left.\left.+\frac{(p t)^{2}}{4}\left[b_{0}^{2}-3 b_{0}+\frac{1}{2}+l_{1}\left(2 b_{0}-1\right)\right]+\frac{1}{\sqrt{1+p^{2} t^{2}}} B_{1}\right)\right]+\mathcal{O}\left(\epsilon^{2}\right),
\end{aligned}
$$

where,

$$
\begin{aligned}
b_{0}= & \log \left(\frac{p t}{2}\right), \quad B_{0}=\log \left(R_{1} R_{2}\right), \quad b_{1}=\log \left(\frac{p t}{2}\right) \log \left(\frac{p^{3} t}{2(1-p)^{2}}\right) \\
B_{1}= & J_{1}^{(2)}+\operatorname{Li}_{2}\left(R_{1}\right)+\frac{1}{4} \log ^{2}\left(R_{1}\right)-\frac{1}{2} \log \left(R_{1} R_{2}\right) \log \left(\frac{p q}{4(1-p)}\right)+\frac{1}{4} \log \left(\frac{q}{4}\right) \log \left(R_{1}\right) \\
& -\frac{1}{8} \log ^{2}\left(\frac{q}{4}\right)-\log ^{2} 2
\end{aligned}
$$

where $J_{1}^{(2)}$ is given in eq. (D.6).

The terms $\sim(p t)$ and $\sim(p t)^{2}$ in (D.18) and (D.19) appear upon replacing $I_{i}^{(2)}(t) \rightarrow \tilde{I}_{i}^{(2)}$ $(i=2,3)$ and $I_{3}^{(j)}(t) \rightarrow \tilde{I}_{3}^{(j)}(j=1,3)$, respectively.

Up to $O\left(\varepsilon^{0}\right)$, the results $(4.42),(4.47)-(4.49)$ and (D.17)-(D.19) are in full agreement with [56]. Here, however, the results (D.17)-(D.19) are given up to $O\left(\varepsilon^{1}\right)$.

\section{E Definitions for elliptic polylogarithms}

We provide here definitions relevant to the all orders results of the main text of the paper.

Results for $\boldsymbol{L}_{\boldsymbol{i}}$ and $\boldsymbol{K}_{\boldsymbol{i}}$. In this section we provide the definitions for the various factors appearing in eqs. (4.43), (4.44) and (4.62). Specifically, the eMPLs expressions for the 
relevant logarithms are defined as,

$$
\begin{aligned}
& L_{1}=E_{4}\left({ }_{-1}^{1} ; x\right)-2 E_{4}\left(\frac{1}{0} ; x\right)+E_{4}\left(\frac{1}{1} ; x\right), \\
& L_{2}=\sum_{i=1}^{4} E_{4}\left({ }_{a_{i}}^{1} ; x\right)-2 E_{4}\left({ }_{-1}^{1} ; x\right)-2 E_{4}(\underset{1}{1} ; x)+\log \left(t^{2}+1\right)-2 \log (t), \\
& L_{3}=-\sum_{i=1}^{4} E_{4}\left(\underset{a_{i}}{1} ; x\right)+3 E_{4}\left({ }_{-1}^{1} ; x\right)-2 E_{4}(\underset{0}{1} ; x)+3 E_{4}(\underset{1}{1} ; x)-\log \left(t^{2}+1\right)+2 \log (t), \\
& L_{4}=\sum_{i=1}^{4} E_{4}\left({ }_{a_{i}}^{1} ; x\right)-E_{4}\left({ }_{-1}^{1} ; x\right)-E_{4}\left({ }_{1}^{1} ; x\right)+\log \left(t^{2}+1\right)-\log (t) \text {, } \\
& L_{5}=-\sum_{i=1}^{4} E_{4}\left(\underset{a_{i}}{1} ; x\right)+3 E_{4}(\underset{-1}{1} ; x)-2 E_{4}(\underset{0}{1} ; x)+3 E_{4}(\underset{1}{1} ; x)-\log \left(t^{2}+1\right), \\
& L_{6}=2 E_{4}\left({ }_{-1}^{1} ; x\right)-2 E_{4}\left(\begin{array}{l}
1 \\
0
\end{array} ; x\right)+2 E_{4}\left({ }_{1}^{1} ; x\right) \text {, } \\
& L_{7}=-\frac{1}{2} \sum_{i=1}^{4} E_{4}\left(\underset{a_{i}}{1} ; x\right)+2 E_{4}(\underset{-1}{1} ; x)-2 E_{4}(\underset{0}{1} ; x)+2 E_{4}\left(\begin{array}{c}
1 \\
1
\end{array} ; x\right)-\frac{1}{2} \log \left(t^{2}+1\right), \\
& L_{8}=-\sum_{i=1}^{4} E_{4}\left(\underset{a_{i}}{1} ; x\right)+3 E_{4}\left({ }_{-1}^{1} ; x\right)-2 E_{4}\left(\begin{array}{l}
1 \\
0
\end{array} ; x\right)+3 E_{4}\left(\begin{array}{l}
1 \\
1
\end{array} ; x\right)-\log \left(t^{2}+1\right)+\log (t) \text {, } \\
& L_{9}=\frac{1}{2} \sum_{i=1}^{4} E_{4}\left(\underset{a_{i}}{1} ; x\right)-E_{4}\left({ }_{-1}^{1} ; x\right)-E_{4}\left(\frac{1}{1} ; x\right)+\frac{1}{2} \log \left(t^{2}+1\right)-\log (t),
\end{aligned}
$$

while the primitives of the relevant integration kernels are defined as,

$$
\begin{aligned}
& K_{1}=E_{4}\left({ }_{-1}^{-1} ; x\right)-E_{4}\left(\begin{array}{c}
-1 \\
1
\end{array} ; x\right)-E_{4}\left({ }_{-1}^{1} ; x\right), \\
& K_{2}=E_{4}\left({ }_{-1}^{-1} ; x\right)-E_{4}\left({ }_{1}^{-1} ; x\right)-E_{4}\left(\frac{1}{1} ; x\right) \text {, } \\
& K_{3}=\sum_{i=1}^{4} E_{4}\left({ }_{a_{i}}^{1} ; x\right)+2 E_{4}\left({ }_{-1}^{-1} ; x\right)+2 E_{4}\left(\begin{array}{c}
-1 \\
1
\end{array} ; x\right)-2 E_{4}\left(\begin{array}{c}
1 \\
-1
\end{array} ; x\right), \\
& K_{4}=E_{4}\left(\begin{array}{c}
-1 \\
-1
\end{array} ; x\right)-E_{4}(\underset{1}{-1} ; x)-E_{4}\left(\begin{array}{l}
1 \\
1
\end{array} ; x\right), \\
& K_{5}=-2 E_{4}(\underset{\infty}{-1} ; x) \text {, } \\
& K_{6}=E_{4}\left({ }_{1}^{1} ; x\right)-E_{4}\left({ }_{-1}^{1} ; x\right) \text {, } \\
& K_{7}=E_{4}\left({ }_{-1}^{-1} ; x\right)-E_{4}\left(\frac{-1}{1} ; x\right)-E_{4}\left(\frac{1}{1} ; x\right) \text {, } \\
& K_{8}=-\sum_{i=1}^{4} E_{4}\left(\underset{a_{i}}{1} ; x\right)-2 E_{4}\left({ }_{-1}^{1} ; x\right)-2 E_{4}\left(\frac{1}{1} ; x\right), \\
& K_{9}=2 E_{4}\left(\begin{array}{c}
-1 \\
-1
\end{array} ; x\right)+2 E_{4}(\underset{1}{-1} ; x)+2 E_{4}\left(\begin{array}{l}
1 \\
1
\end{array} ; x\right) .
\end{aligned}
$$

For the sunrises $\hat{J}_{1,1,2}^{\text {reg }}$ and $\hat{J}_{1,1,1}^{\text {reg }}$ we have (see eq. (4.62)):

$$
\begin{aligned}
K_{31} & =\frac{1}{c_{4}} E_{4}\left(\begin{array}{l}
0 \\
0
\end{array} ; x\right), \\
K_{21} & =\frac{1}{c_{4}} E_{4}\left(\begin{array}{l}
0 \\
0
\end{array} ; x\right)-K_{21}^{(a)}-K_{21}^{(b)}, \\
K_{21}^{(a)} & =\frac{s_{1}}{2} E_{4}(\underset{\infty}{-1} ; x)-\left(\frac{s_{2}}{6 c_{4}}+\frac{4 c_{4} \eta_{1}}{\omega_{1}}\right) E_{4}\left({ }_{0}^{0} ; x\right), \quad K_{21}^{(b)}=c_{4} \hat{Z}_{4}(x),
\end{aligned}
$$


where,

$$
\hat{Z}_{4}(x) * L_{a}^{i}(x) \equiv \int d x \Phi_{4}(x) L_{a}^{i}(x)=Z_{4}(x) \star L_{a}^{i}(x),
$$

where the definition of the product of the $\star$-operator for a eMPL is provided in the next subsection.

Definition of the $\star$-operator. In order to obtain an eMPLs expression with the $\star$ operator we need to perform integrals of the form:

$$
Z_{4}(x) \star E_{4}\left(\begin{array}{c}
a_{1} a_{2} \ldots \\
b_{1} b_{2} \ldots
\end{array} ; x\right) \equiv \int d x \Phi_{4}(x) E_{4}\left(\begin{array}{c}
a_{1} a_{2} \ldots \\
b_{1} b_{2} \ldots
\end{array} ; x\right) .
$$

When $E_{4}=1$, the $\star$-operator is trivial (see eq. (4.9)). For more complicated cases, we can use the integration by parts. We have,

$$
\begin{aligned}
\int d x \Phi_{4}(x) E_{4}\left(\begin{array}{c}
a_{1} a_{2} \ldots \\
b_{1} b_{2} \ldots
\end{array} ; x\right) & =Z_{4}(x) E_{4}\left(\begin{array}{c}
a_{1} a_{2} \ldots \\
b_{1} b_{2} \ldots
\end{array} ; x\right)-\int d x Z_{4}(x) \frac{\partial}{\partial x} E_{4}\left(\begin{array}{c}
a_{1} a_{2} \ldots \\
b_{1} b_{2} \ldots
\end{array} ; x\right) \\
& =Z_{4}(x) E_{4}\left(\begin{array}{c}
a_{1} a_{2} \ldots \\
b_{1} b_{2} \ldots
\end{array} ; x\right)-\int d x Z_{4}(x) \phi_{a_{1}}\left(b_{1}, x\right) E_{4}\left(\begin{array}{c}
a_{2} \ldots \\
b_{2} \ldots
\end{array} ; x\right) .
\end{aligned}
$$

By noticing that $Z_{4}^{(1)}(x)=Z_{4}(x)$ and that $Z_{4}^{(n)}(x)$ appears only in the last integration (see discussion in the main text after eq. (4.9)), we have the following possible cases.

1. Let $a_{1}=b_{1}=0$, then,

$$
Z_{4}(x) \phi_{0}(0, x)=\frac{c_{4} Z_{4}(x)}{y(x)}=\phi_{1}(\infty, x)
$$

and, thus, the last term in r.h.s. of (E.6) evaluates to,

$$
\int d x \phi_{1}(\infty, x) E_{4}\left(\begin{array}{c}
a_{2} \ldots \\
b_{2} \ldots
\end{array} ; x\right)=E_{4}\left(\begin{array}{cc}
1 & a_{2} \ldots \\
\infty & b_{2} \ldots
\end{array} ; x\right) .
$$

2. Let $a_{1}=1$ and $b_{1}=c$, where $c$ is some constant. Then we have,

$$
Z_{4}(x) \phi_{1}(c, x)=\frac{Z_{4}(x)}{x-c}=\phi_{2}(c, x)+\Phi_{4}(x) .
$$

Thus, for the last term in r.h.s. of (E.6) we have the following results,

$$
\int d x Z_{4}(x) \phi_{1}(c, x) E_{4}\left(\begin{array}{l}
a_{2} \ldots \\
b_{2} \ldots
\end{array} ; x\right)=E_{4}\left(\begin{array}{l}
2 a_{2} \ldots \\
c b_{2} \ldots
\end{array} ; x\right)+\int d x \Phi_{4}(x) E_{4}\left(\begin{array}{l}
a_{2} \ldots \\
b_{2} \ldots
\end{array} ; x\right) .
$$

3. Let $a_{1}=-1$ and $b_{1}=c$, where $c$ is also some constant. Then we have,

$$
Z_{4}(x) \phi_{-1}(c, x)=\frac{Z_{4}(x) y(c)}{(x-c) y(x)}-\left(\delta_{c}^{0}+\delta_{c}^{1}\right) \frac{Z_{4}(x)}{x-c},
$$

where $\delta_{c}^{0}$ and $\delta_{c}^{1}$ are Cronecker symbols. Considering the results (4.4) with $Z_{4}^{(n)}(x)$, we have,

$$
Z_{4}(x) \phi_{-1}(c, x)=\phi_{-2}(c, x)-\left(\delta_{c}^{0}+\delta_{c}^{1}\right)\left[\phi_{2}(c, x)+\Phi_{4}(x)\right]
$$


Thus, for the last term in r.h.s. of (E.6) we have the following results,

$$
\begin{aligned}
& \int d x Z_{4}(x) \phi_{-1}(c, x) E_{4}\left(\begin{array}{c}
a_{2} \ldots \\
b_{2} \ldots
\end{array} ; x\right) \\
& \quad=E_{4}\left(\begin{array}{c}
-2 a_{2} \ldots \\
c b_{2} \ldots
\end{array} ; x\right)-\left(\delta_{c}^{0}+\delta_{c}^{1}\right)\left[E_{4}\left(\begin{array}{c}
2 a_{2} \ldots \\
c b_{2} \ldots
\end{array} ; x\right)+\int d x \Phi_{4}(x) E_{4}\left(\begin{array}{c}
a_{2} \ldots \\
b_{2} \ldots
\end{array} ; x\right)\right] .
\end{aligned}
$$

Therefore, in cases $\mathbf{2}$ and $\mathbf{3}$ we expressed the initial integral (E.5) as a combination of eMPLs and integrals of the form (E.5) with lower depth. Therefore these integrals can be expressed fully in terms of eMPLs by iterating the application of the IBP identities (E.6) and by using the definition of $Z_{4}(x)$, eq. (4.9).

4. Let $a_{1}=-1$ and $b_{1}=\infty$, then we have,

$$
Z_{4}(x) \phi_{-1}(\infty, x)=\frac{x Z_{4}(x)}{y(x)}=\phi_{-2}(\infty, x)+\frac{1}{c_{4}} .
$$

Thus, for the last term in r.h.s. of (E.6) we have the following results,

$$
\int d x Z_{4}(x) \phi_{-1}(\infty, x) E_{4}\left(\begin{array}{c}
a_{2} \ldots \\
b_{2} \ldots
\end{array} ; x\right)=E_{4}\left(\begin{array}{cc}
-2 & a_{2} \ldots \\
\infty & b_{2} \ldots
\end{array} ; x\right)+\frac{1}{c_{4}} \int d x E_{4}\left(\begin{array}{c}
a_{2} \ldots \\
b_{2} \ldots
\end{array} ; x\right) .
$$

The last term in the r.h.s. can be transformed by integration by part as,

$$
\int d x E_{4}\left(\begin{array}{c}
a_{2} \ldots \\
b_{2} \ldots
\end{array} ; x\right)=(x+d) E_{4}\left(\begin{array}{c}
a_{2} \ldots \\
b_{2} \ldots
\end{array} ; x\right)-\int d x(x+d) \phi_{a_{2}}\left(b_{2}, x\right) E_{4}\left(\begin{array}{l}
a_{2} \ldots \\
b_{2} \ldots
\end{array} ; x\right),
$$

where $d$ is arbitrary constant.

Finally, the following cases need to be considered.

4.1. Let $a_{2}=b_{2}=0$, then in the case $d=0$ we have,

$$
\frac{c_{4} x}{y(x)}=\phi_{-1}(\infty, x)
$$

and the last term in r.h.s. of (E.16) evaluates to,

$$
\int d x E_{4}\left(\begin{array}{cc}
0 & a_{3} \ldots \\
0 & b_{3} \ldots
\end{array} ; x\right)=E_{4}\left(\begin{array}{ccc}
-1 & 0 & a_{3} \ldots \\
\infty & 0 & b_{3} \ldots
\end{array} ; x\right) .
$$

4.2. Let $a_{2}=1$ and $b_{2}=c_{1}$, where $c_{1}$ is some constant. Then, we have, when $d=-c_{1}$,

$$
\frac{x+d}{x-c_{1}}=1 .
$$

Then, the last term in r.h.s. of (E.16) has the following form,

$$
\int d x E_{4}\left(\begin{array}{cc}
1 & a_{3} \ldots \\
c_{1} b_{3} \ldots
\end{array} ; x\right)=\left(x-c_{1}\right) E_{4}\left(\begin{array}{c}
1 a_{3} \ldots \\
c_{1} b_{3} \ldots
\end{array} ; x\right)-\int d x E_{4}\left(\begin{array}{l}
a_{3} \ldots \\
b_{3} \ldots
\end{array} ; x\right) .
$$

As in cases $\mathbf{2}$ and $\mathbf{3}$ we expressed reduced integrals in the r.h.s. of (E.16) as a combination of eMPLs and lower depth integrals. The iterative application of the IBP procedure (E.6) terminates with,

$$
\int d x=x+d_{1}
$$

where $d$ is arbitrary constant. 
4.3. Let $a_{2}=-1$ and $b_{2}=c_{2}$, where $c_{1}$ is some constant. Then, we have, when $d=-c_{2}$,

$$
\frac{x+d}{\left(x-c_{2}\right) y(x)}=\frac{1}{y(x)}=\frac{1}{c_{4}} \phi_{0}(0, x),
$$

and, thus, the last term in r.h.s. of (E.16) has the closed form,

$$
\int d x E_{4}\left(\begin{array}{c}
-1 a_{3} \ldots \\
c_{2} b_{3} \ldots
\end{array} ; x\right)=\left(x-c_{2}\right) E_{4}\left(\begin{array}{c}
-1 a_{3} \ldots \\
c_{2} b_{3} \ldots
\end{array} ; x\right)--\frac{1}{c_{4}} E_{4}\left(\begin{array}{c}
0 a_{3} \ldots \\
0 b_{3} \ldots
\end{array} ; x\right) .
$$

4.4. Let $a_{2}=-1$ and $b_{2}=\infty$, then in the case $d=0$, we have,

$$
\frac{(x+d) x}{y(x)}=\frac{x^{2}}{y(x)}=c_{4} \Phi_{4}(x)+\frac{s_{1}}{2} \frac{x}{y(x)}-\frac{s_{2}}{6} \frac{1}{y(x)}-4 c_{4}^{2} \frac{\eta_{1}}{\omega_{1}} \frac{1}{y(x)},
$$

and, thus, the last term in r.h.s. of (E.16) has the following form,

$$
\begin{aligned}
& \int d x E_{4}\left(\begin{array}{ccc}
-1 & a_{3} \ldots \\
\infty & b_{3} \ldots
\end{array} ; x\right)=\left(x+\frac{s_{1}}{2}\right) E_{4}\left(\begin{array}{ccc}
-1 & a_{3} \ldots \\
\infty & b_{3} \ldots
\end{array} ; x\right)-\left(\frac{s_{2}}{6}+4 c_{4}^{2} \frac{\eta_{1}}{\omega_{1}}\right) E_{4}\left(\begin{array}{ccc}
0 & a_{3} \ldots \\
0 & b_{3} \ldots
\end{array} ; x\right) \\
& +c_{4} \int d x \Phi(x) E_{4}\left(\begin{array}{c}
a_{3} \ldots \\
b_{3} \ldots
\end{array} ; x\right) \text {. }
\end{aligned}
$$

Therefore, we reduced any integral of the form (E.5) to the computation of integrals of the form (E.5) with lower depth. Iterating (E.6) several times and using (4.9) we can compute any integral of the form (E.5) in terms of eMPLs.

Open Access. This article is distributed under the terms of the Creative Commons Attribution License (CC-BY 4.0), which permits any use, distribution and reproduction in any medium, provided the original author(s) and source are credited.

\section{References}

[1] A.B. Goncharov, Multiple polylogarithms, cyclotomy and modular complexes, Math. Res. Lett. 5 (1998) 497 [arXiv:1105.2076] [INSPIRE].

[2] E. Remiddi and J.A.M. Vermaseren, Harmonic polylogarithms, Int. J. Mod. Phys. A 15 (2000) 725 [hep-ph/9905237] [INSPIRE].

[3] A.A. Sabry, Fourth order spectral functions for the electron propagato, Nucl. Phys. 33 (1962) 401.

[4] D.J. Broadhurst, J. Fleischer and O.V. Tarasov, Two loop two point functions with masses: Asymptotic expansions and Taylor series, in any dimension, Z. Phys. C 60 (1993) 287 [hep-ph/9304303] [INSPIRE].

[5] S. Laporta and E. Remiddi, Analytic treatment of the two loop equal mass sunrise graph, Nucl. Phys. B 704 (2005) 349 [hep-ph/0406160] [InSPIRE].

[6] B.A. Kniehl, A.V. Kotikov, A. Onishchenko and O. Veretin, Two-loop sunset diagrams with three massive lines, Nucl. Phys. B 738 (2006) 306 [hep-ph/0510235] [InSPIRE].

[7] D.H. Bailey, J.M. Borwein, D. Broadhurst and M.L. Glasser, Elliptic integral evaluations of Bessel moments, J. Phys. A 41 (2008) 205203 [arXiv:0801.0891] [InSPIRE]. 
[8] S. Müller-Stach, S. Weinzierl and R. Zayadeh, A Second-Order Differential Equation for the Two-Loop Sunrise Graph with Arbitrary Masses, Commun. Num. Theor. Phys. 6 (2012) 203 [arXiv:1112.4360] [INSPIRE].

[9] L. Adams, C. Bogner and S. Weinzierl, The two-loop sunrise graph with arbitrary masses, J. Math. Phys. 54 (2013) 052303 [arXiv: 1302.7004] [INSPIRE].

[10] S. Bloch and P. Vanhove, The elliptic dilogarithm for the sunset graph, J. Number Theor. 148 (2015) 328 [arXiv:1309.5865] [inSPIRE].

[11] E. Remiddi and L. Tancredi, Schouten identities for Feynman graph amplitudes; The Master Integrals for the two-loop massive sunrise graph, Nucl. Phys. B 880 (2014) 343 [arXiv:1311.3342] [INSPIRE].

[12] L. Adams, C. Bogner and S. Weinzierl, The two-loop sunrise graph in two space-time dimensions with arbitrary masses in terms of elliptic dilogarithms, J. Math. Phys. $\mathbf{5 5}$ (2014) 102301 [arXiv:1405.5640] [INSPIRE].

[13] L. Adams, C. Bogner and S. Weinzierl, The two-loop sunrise integral around four space-time dimensions and generalisations of the Clausen and Glaisher functions towards the elliptic case, J. Math. Phys. 56 (2015) 072303 [arXiv:1504.03255] [inSPIRE].

[14] L. Adams, C. Bogner and S. Weinzierl, The iterated structure of the all-order result for the two-loop sunrise integral, J. Math. Phys. 57 (2016) 032304 [arXiv:1512.05630] [INSPIRE].

[15] S. Bloch, M. Kerr and P. Vanhove, Local mirror symmetry and the sunset Feynman integral, Adv. Theor. Math. Phys. 21 (2017) 1373 [arXiv:1601.08181] [InSPIRE].

[16] L. Adams and S. Weinzierl, Feynman integrals and iterated integrals of modular forms, Commun. Num. Theor. Phys. 12 (2018) 193 [arXiv:1704.08895] [INSPIRE].

[17] C. Bogner, A. Schweitzer and S. Weinzierl, Analytic continuation and numerical evaluation of the kite integral and the equal mass sunrise integral, Nucl. Phys. B 922 (2017) 528 [arXiv: 1705.08952] [INSPIRE].

[18] L. Adams and S. Weinzierl, The $\varepsilon$-form of the differential equations for Feynman integrals in the elliptic case, Phys. Lett. B 781 (2018) 270 [arXiv:1802.05020] [INSPIRE].

[19] I. Hönemann, K. Tempest and S. Weinzierl, Electron self-energy in QED at two loops revisited, Phys. Rev. D 98 (2018) 113008 [arXiv:1811.09308] [InSPIRE].

[20] S. Bloch, M. Kerr and P. Vanhove, A Feynman integral via higher normal functions, Compos. Math. 151 (2015) 2329 [arXiv:1406.2664] [INSPIRE].

[21] M. Søgaard and Y. Zhang, Elliptic Functions and Maximal Unitarity, Phys. Rev. D 91 (2015) 081701 [arXiv:1412.5577] [INSPIRE].

[22] L. Tancredi, Integration by parts identities in integer numbers of dimensions. A criterion for decoupling systems of differential equations, Nucl. Phys. B 901 (2015) 282 [arXiv: 1509. 03330] [INSPIRE].

[23] A. Primo and L. Tancredi, On the maximal cut of Feynman integrals and the solution of their differential equations, Nucl. Phys. B 916 (2017) 94 [arXiv:1610.08397] [INSPIRE].

[24] E. Remiddi and L. Tancredi, Differential equations and dispersion relations for Feynman amplitudes. The two-loop massive sunrise and the kite integral, Nucl. Phys. B 907 (2016) 400 [arXiv: 1602.01481] [INSPIRE]. 
[25] L. Adams, C. Bogner, A. Schweitzer and S. Weinzierl, The kite integral to all orders in terms of elliptic polylogarithms, J. Math. Phys. 57 (2016) 122302 [arXiv:1607.01571] [INSPIRE].

[26] R. Bonciani, V. Del Duca, H. Frellesvig, J.M. Henn, F. Moriello and V.A. Smirnov, Two-loop planar master integrals for Higgs $\rightarrow 3$ partons with full heavy-quark mass dependence, JHEP 12 (2016) 096 [arXiv: 1609.06685] [INSPIRE].

[27] A. von Manteuffel and L. Tancredi, A non-planar two-loop three-point function beyond multiple polylogarithms, JHEP 06 (2017) 127 [arXiv:1701.05905] [INSPIRE].

[28] L. Adams, E. Chaubey and S. Weinzierl, Simplifying Differential Equations for Multiscale Feynman Integrals beyond Multiple Polylogarithms, Phys. Rev. Lett. 118 (2017) 141602 [arXiv: 1702 . 04279] [INSPIRE].

[29] J. Ablinger et al., Iterated Elliptic and Hypergeometric Integrals for Feynman Diagrams, J. Math. Phys. 59 (2018) 062305 [arXiv: 1706.01299] [InSPIRE].

[30] A. Primo and L. Tancredi, Maximal cuts and differential equations for Feynman integrals. An application to the three-loop massive banana graph, Nucl. Phys. B 921 (2017) 316 [arXiv: 1704.05465] [INSPIRE].

[31] G. Passarino, Elliptic Polylogarithms and Basic Hypergeometric Functions, Eur. Phys. J. C 77 (2017) 77 [arXiv:1610.06207] [inSPIRE].

[32] E. Remiddi and L. Tancredi, An Elliptic Generalization of Multiple Polylogarithms, Nucl. Phys. B 925 (2017) 212 [arXiv:1709.03622] [InSPIRE].

[33] J.L. Bourjaily, A.J. McLeod, M. Spradlin, M. von Hippel and M. Wilhelm, Elliptic Double-Box Integrals: Massless Scattering Amplitudes beyond Polylogarithms, Phys. Rev. Lett. 120 (2018) 121603 [arXiv:1712.02785] [INSPIRE].

[34] M. Hidding and F. Moriello, All orders structure and efficient computation of linearly reducible elliptic Feynman integrals, JHEP 01 (2019) 169 [arXiv:1712.04441] [INSPIRE].

[35] J. Broedel, C. Duhr, F. Dulat and L. Tancredi, Elliptic polylogarithms and iterated integrals on elliptic curves. Part I: general formalism, JHEP 05 (2018) 093 [arXiv: 1712.07089] [INSPIRE].

[36] J. Broedel, C. Duhr, F. Dulat and L. Tancredi, Elliptic polylogarithms and iterated integrals on elliptic curves II: an application to the sunrise integral, Phys. Rev. D 97 (2018) 116009 [arXiv: 1712.07095] [INSPIRE].

[37] J. Broedel, C. Duhr, F. Dulat, B. Penante and L. Tancredi, Elliptic symbol calculus: from elliptic polylogarithms to iterated integrals of Eisenstein series, JHEP 08 (2018) 014 [arXiv: 1803.10256] [INSPIRE].

[38] R.N. Lee, A.V. Smirnov and V.A. Smirnov, Solving differential equations for Feynman integrals by expansions near singular points, JHEP 03 (2018) 008 [arXiv:1709.07525] [INSPIRE].

[39] R.N. Lee, A.V. Smirnov and V.A. Smirnov, Evaluating 'elliptic' master integrals at special kinematic values: using differential equations and their solutions via expansions near singular points, JHEP 07 (2018) 102 [arXiv: 1805.00227] [INSPIRE].

[40] L. Adams, E. Chaubey and S. Weinzierl, Planar Double Box Integral for Top Pair Production with a Closed Top Loop to all orders in the Dimensional Regularization Parameter, Phys. Rev. Lett. 121 (2018) 142001 [arXiv:1804.11144] [INSPIRE]. 
[41] L. Adams, E. Chaubey and S. Weinzierl, Analytic results for the planar double box integral relevant to top-pair production with a closed top loop, JHEP 10 (2018) 206 [arXiv: 1806.04981] [INSPIRE].

[42] J. Broedel, C. Duhr, F. Dulat, B. Penante and L. Tancredi, Elliptic Feynman integrals and pure functions, JHEP 01 (2019) 023 [arXiv: 1809.10698] [INSPIRE].

[43] J.L. Bourjaily, A.J. McLeod, M. von Hippel and M. Wilhelm, Bounded Collection of Feynman Integral Calabi-Yau Geometries, Phys. Rev. Lett. 122 (2019) 031601 [arXiv: 1810.07689] [INSPIRE].

[44] J.L. Bourjaily, A.J. McLeod, M. von Hippel and M. Wilhelm, Rationalizing Loop Integration, JHEP 08 (2018) 184 [arXiv:1805.10281] [INSPIRE].

[45] M. Besier, D. Van Straten and S. Weinzierl, Rationalizing roots: an algorithmic approach, Commun. Num. Theor. Phys. 13 (2019) 253 [arXiv:1809.10983] [InSPIRE].

[46] P. Mastrolia and S. Mizera, Feynman Integrals and Intersection Theory, JHEP 02 (2019) 139 [arXiv: 1810.03818] [INSPIRE].

[47] J. Ablinger, J. Blümlein, P. Marquard, N. Rana and C. Schneider, Automated Solution of First Order Factorizable Systems of Differential Equations in One Variable, Nucl. Phys. B 939 (2019) 253 [arXiv:1810.12261] [INSPIRE].

[48] H. Frellesvig et al., Decomposition of Feynman Integrals on the Maximal Cut by Intersection Numbers, JHEP 05 (2019) 153 [arXiv:1901.11510] [INSPIRE].

[49] H. Frellesvig, M. Hidding, L. Maestri, F. Moriello and G. Salvatori, The complete set of two-loop master integrals for Higgs + jet production in QCD, JHEP 06 (2020) 093 [arXiv: 1911.06308] [INSPIRE].

[50] R. Bonciani et al., Evaluating a family of two-loop non-planar master integrals for Higgs + jet production with full heavy-quark mass dependence, JHEP 01 (2020) 132 [arXiv: 1907.13156] [INSPIRE].

[51] F. Moriello, Generalised power series expansions for the elliptic planar families of Higgs + jet production at two loops, JHEP 01 (2020) 150 [arXiv:1907.13234] [INSPIRE].

[52] J. Broedel, C. Duhr, F. Dulat, B. Penante and L. Tancredi, Elliptic polylogarithms and Feynman parameter integrals, JHEP 05 (2019) 120 [arXiv: 1902.09971] [INSPIRE].

[53] J. Blümlein, Large scale analytic calculations in quantum field theories, arXiv:1905.02148 [INSPIRE].

[54] J. Broedel and A. Kaderli, Functional relations for elliptic polylogarithms, J. Phys. A 53 (2020) 245201 [arXiv: 1906.11857] [inSPIRE].

[55] C. Bogner, S. Müller-Stach and S. Weinzierl, The unequal mass sunrise integral expressed through iterated integrals on $\overline{\mathcal{M}}_{1,3}$, Nucl. Phys. B 954 (2020) 114991 [arXiv:1907.01251] [INSPIRE].

[56] B.A. Kniehl, A.V. Kotikov, A.I. Onishchenko and O.L. Veretin, Two-loop diagrams in non-relativistic QCD with elliptics, Nucl. Phys. B 948 (2019) 114780 [arXiv:1907.04638] [INSPIRE].

[57] J. Broedel, C. Duhr, F. Dulat, R. Marzucca, B. Penante and L. Tancredi, An analytic solution for the equal-mass banana graph, JHEP 09 (2019) 112 [arXiv:1907.03787] [INSPIRE]. 
[58] S. Abreu, M. Becchetti, C. Duhr and R. Marzucca, Three-loop contributions to the $\rho$ parameter and iterated integrals of modular forms, JHEP 02 (2020) 050

[arXiv: 1912.02747] [INSPIRE].

[59] C. Duhr and L. Tancredi, Algorithms and tools for iterated Eisenstein integrals, JHEP 02 (2020) 105 [arXiv: 1912.00077] [INSPIRE].

[60] A. Klemm, C. Nega and R. Safari, The l-loop Banana Amplitude from GKZ Systems and relative Calabi-Yau Periods, JHEP 04 (2020) 088 [arXiv: 1912.06201] [INSPIRE].

[61] K. Bönisch, F. Fischbach, A. Klemm, C. Nega and R. Safari, Analytic structure of all loop banana integrals, JHEP 05 (2021) 066 [arXiv: 2008.10574] [INSPIRE].

[62] M. Walden and S. Weinzierl, Numerical evaluation of iterated integrals related to elliptic Feynman integrals, Comput. Phys. Commun. 265 (2021) 108020 [arXiv:2010.05271] [INSPIRE].

[63] M. Bezuglov, Calculation of master integrals in terms of elliptic multiple polylogarithms, Int. J. Mod. Phys. A 35 (2020) 2050063 [arXiv:2003.05367] [inSPIRE].

[64] M.A. Bezuglov, A.I. Onishchenko and O.L. Veretin, Massive kite diagrams with elliptics, Nucl. Phys. B 963 (2021) 115302 [arXiv:2011.13337] [INSPIRE].

[65] R.N. Lee and A.I. Onishchenko, Master integrals for bipartite cuts of three-loop photon self energy, JHEP 04 (2021) 177 [arXiv: 2012.04230] [INSPIRE].

[66] S. Weinzierl, Iterated integrals related to Feynman integrals associated to elliptic curves, in Antidifferentiation and the Calculation of Feynman Amplitudes, (2020) [arXiv: 2012.08429] [INSPIRE].

[67] M.A. Bezuglov, Integral representation for three-loop banana graph, arXiv:2104.14681 [INSPIRE].

[68] F.C. Brown and L. Andrey, Multiple Elliptic Polylogarithms, arXiv:1110.6917.

[69] A. Beilinson and A. Levin, The Elliptic Polylogarithms, Proc. of Symp. in Pure Math. 55 Part II (1994) 123.

[70] A. Levin and G. Racinet, Towards multiple elliptic polylogarithms, math/0703237.

[71] M.Y. Kalmykov and B.A. Kniehl, Towards all-order Laurent expansion of generalized hypergeometric functions around rational values of parameters, Nucl. Phys. B 809 (2009) 365 [arXiv:0807.0567] [INSPIRE].

[72] W.-L. Sang, F. Feng, Y. Jia and S.-R. Liang, Next-to-next-to-leading-order QCD corrections to $\chi_{c 0,2} \rightarrow \gamma \gamma$, Phys. Rev. D 94 (2016) 111501 [arXiv:1511.06288] [INSPIRE].

[73] L.-B. Chen, Y. Liang and C.-F. Qiao, Two-Loop integrals for CP-even heavy quarkonium production and decays, JHEP 06 (2017) 025 [arXiv: 1703.03929] [INSPIRE].

[74] F. Feng, Y. Jia and W.-L. Sang, Next-to-Next-to-Leading-Order QCD Corrections to the Hadronic width of Pseudoscalar Quarkonium, Phys. Rev. Lett. 119 (2017) 252001 [arXiv: 1707.05758] [INSPIRE].

[75] L.-B. Chen, Y. Liang and C.-F. Qiao, NNLO QCD corrections to $\gamma+\eta_{c}\left(\eta_{b}\right)$ exclusive production in electron-positron collision, JHEP 01 (2018) 091 [arXiv:1710.07865] [INSPIRE]. 
[76] L.-B. Chen, J. Jiang and C.-F. Qiao, Two-Loop integrals for CP-even heavy quarkonium production and decays: Elliptic Sectors, JHEP 04 (2018) 080 [arXiv:1712.03516] [INSPIRE].

[77] L. Yang, W.-L. Sang, H.-F. Zhang, Y.-D. Zhang and M.-Z. Zhou, Study on $\eta_{c 2}\left(\eta_{b 2}\right)$ electromagnetic decay into double photons, Phys. Rev. D 103 (2021) 034018 [arXiv:2010.14364] [INSPIRE].

[78] R. Bonciani, A. Ferroglia, T. Gehrmann and C. Studerus, Two-Loop Planar Corrections to Heavy-Quark Pair Production in the Quark-Antiquark Channel, JHEP 08 (2009) 067 [arXiv:0906.3671] [INSPIRE].

[79] M. Czakon, A. Mitov and G.F. Sterman, Threshold Resummation for Top-Pair Hadroproduction to Next-to-Next-to-Leading Log, Phys. Rev. D 80 (2009) 074017 [arXiv:0907.1790] [INSPIRE].

[80] R. Bonciani, A. Ferroglia, T. Gehrmann, A. von Manteuffel and C. Studerus, Two-Loop Leading Color Corrections to Heavy-Quark Pair Production in the Gluon Fusion Channel, JHEP 01 (2011) 102 [arXiv:1011.6661] [INSPIRE].

[81] M. Beneke, P. Falgari, S. Klein and C. Schwinn, Hadronic top-quark pair production with NNLL threshold resummation, Nucl. Phys. B 855 (2012) 695 [arXiv:1109.1536] [INSPIRE].

[82] M. Cacciari, M. Czakon, M. Mangano, A. Mitov and P. Nason, Top-pair production at hadron colliders with next-to-next-to-leading logarithmic soft-gluon resummation, Phys. Lett. B 710 (2012) 612 [arXiv:1111.5869] [INSPIRE].

[83] M. Czakon, P. Fiedler and A. Mitov, Total Top-Quark Pair-Production Cross Section at Hadron Colliders Through $O\left(\alpha_{S}^{4}\right)$, Phys. Rev. Lett. 110 (2013) 252004 [arXiv:1303.6254] [INSPIRE].

[84] R. Bonciani, A. Ferroglia, T. Gehrmann, A. von Manteuffel and C. Studerus, Light-quark two-loop corrections to heavy-quark pair production in the gluon fusion channel, JHEP 12 (2013) 038 [arXiv: 1309.4450] [INSPIRE].

[85] M. Beneke, Y. Kiyo, P. Marquard, A. Penin, J. Piclum and M. Steinhauser, Next-to-Next-to-Next-to-Leading Order QCD Prediction for the Top Antitop S-Wave Pair Production Cross Section Near Threshold in $e^{+} e^{-}$Annihilation, Phys. Rev. Lett. 115 (2015) 192001 [arXiv:1506.06864] [INSPIRE].

[86] M. Czakon, D. Heymes and A. Mitov, High-precision differential predictions for top-quark pairs at the LHC, Phys. Rev. Lett. 116 (2016) 082003 [arXiv:1511.00549] [INSPIRE].

[87] M. Czakon, D. Heymes, A. Mitov, D. Pagani, I. Tsinikos and M. Zaro, Top-pair production at the LHC through NNLO QCD and NLO EW, JHEP 10 (2017) 186 [arXiv:1705.04105] [INSPIRE].

[88] M. Beneke, A. Maier, T. Rauh and P. Ruiz-Femenia, Non-resonant and electroweak NNLO correction to the $e^{+} e^{-}$top anti-top threshold, JHEP 02 (2018) 125 [arXiv:1711.10429] [INSPIRE].

[89] L.-B. Chen and J. Wang, Master integrals of a planar double-box family for top-quark pair production, Phys. Lett. B 792 (2019) 50 [arXiv: 1903.04320] [INSPIRE].

[90] M. Becchetti, R. Bonciani, V. Casconi, A. Ferroglia, S. Lavacca and A. von Manteuffel, Master Integrals for the two-loop, non-planar QCD corrections to top-quark pair production in the quark-annihilation channel, JHEP 08 (2019) 071 [arXiv: 1904.10834] [INSPIRE]. 
[91] S. Di Vita, T. Gehrmann, S. Laporta, P. Mastrolia, A. Primo and U. Schubert, Master integrals for the NNLO virtual corrections to $q \bar{q} \rightarrow t \bar{t}$ scattering in $Q C D$ : the non-planar graphs, JHEP 06 (2019) 117 [arXiv:1904.10964] [INSPIRE].

[92] R. Bonciani, F. Buccioni, N. Rana and A. Vicini, NNLO mixed EW-QCD corrections to single vector boson production, in 14th International Symposium on Radiative Corrections: Application of Quantum Field Theory to Phenomenology, (2019), DOI [arXiv:1912.10951] [INSPIRE].

[93] A.M. Cooper-Sarkar, M. Czakon, M.A. Lim, A. Mitov and A.S. Papanastasiou,

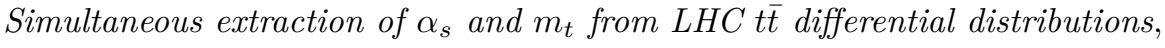
arXiv: 2010.04171 [INSPIRE].

[94] R. Bonciani, F. Buccioni, N. Rana and A. Vicini, Next-to-Next-to-Leading Order Mixed QCD-Electroweak Corrections to on-Shell Z Production, Phys. Rev. Lett. 125 (2020) 232004 [arXiv: 2007.06518] [INSPIRE].

[95] B.A. Kniehl and A.A. Penin, Order alpha**3 ln (1/alpha) corrections to positronium decays, Phys. Rev. Lett. 85 (2000) 1210 [Erratum ibid. 85 (2000) 3065] [hep-ph/0004267] [INSPIRE].

[96] G.S. Adkins, N.M. McGovern, R.N. Fell and J. Sapirstein, Two loop corrections to the decay rate of parapositronium, Phys. Rev. A 68 (2003) 032512 [hep-ph/0305251] [INSPIRE].

[97] A. Czarnecki, K. Melnikov and A. Yelkhovsky, alpha**2 corrections to parapositronium decay, Phys. Rev. Lett. 83 (1999) 1135 [hep-ph/9904478] [inSPIRE].

[98] A. Czarnecki, K. Melnikov and A. Yelkhovsky, $\alpha^{2}$ corrections to parapositronium decay: A detailed description, Phys. Rev. A 61 (2000) 052502 [hep-ph/9910488] [INSPIRE].

[99] B.A. Kniehl, A.V. Kotikov and O.L. Veretin, Orthopositronium lifetime: Analytic results in $O(\alpha)$ and $O\left(\alpha^{3} \ln \alpha\right)$, Phys. Rev. Lett. 101 (2008) 193401 [arXiv:0806.4927] [INSPIRE].

[100] B.A. Kniehl, A.V. Kotikov and O.L. Veretin, Orthopositronium lifetime at $O(\alpha)$ and $O\left(\alpha^{3} \ln \alpha\right)$ in closed form, Phys. Rev. A 80 (2009) 052501 [arXiv:0909.1431] [INSPIRE].

[101] B.A. Kniehl, A.V. Kotikov and O.L. Veretin, Irrational constants in positronium decays, in 9th DESY Workshop on Elementary Particle Theory: Loops and Legs in Quantum Field Theory, (2008) [arXiv: 0811.0306] [INSPIRE].

[102] A.V. Kotikov, Differential equations method: New technique for massive Feynman diagrams calculation, Phys. Lett. B 254 (1991) 158 [INSPIRE].

[103] A.V. Kotikov, Differential equations method: The calculation of vertex type Feynman diagrams, Phys. Lett. B 259 (1991) 314 [InSPIRE].

[104] A.V. Kotikov, Differential equation method: The calculation of $N$ point Feynman diagrams, Phys. Lett. B 267 (1991) 123 [Erratum ibid. 295 (1992) 409] [INSPIRE].

[105] Z. Bern, L.J. Dixon and D.A. Kosower, Dimensionally regulated pentagon integrals, Nucl. Phys. B 412 (1994) 751 [hep-ph/9306240] [INSPIRE].

[106] E. Remiddi, Differential equations for Feynman graph amplitudes, Nuovo Cim. A 110 (1997) 1435 [hep-th/9711188] [INSPIRE].

[107] A.V. Kotikov, New method of massive Feynman diagrams calculation, Mod. Phys. Lett. A 6 (1991) 677 [INSPIRE]. 
[108] B.A. Kniehl and A.V. Kotikov, Counting master integrals: integration-by-parts procedure with effective mass, Phys. Lett. B 712 (2012) 233 [arXiv:1202.2242] [INSPIRE].

[109] A.V. Kotikov, About calculation of massless and massive Feynman integrals, Particles $\mathbf{3}$ (2020) 394 [arXiv: 2004.06625] [INSPIRE].

[110] A.V. Kotikov, Differential equations and Feynman integrals, in Antidifferentiation and the Calculation of Feynman Amplitudes, (2021) [arXiv:2102.07424] [INSPIRE].

[111] O.V. Tarasov, Hypergeometric representation of the two-loop equal mass sunrise diagram, Phys. Lett. B 638 (2006) 195 [hep-ph/0603227] [INSPIRE].

[112] B.A. Kniehl, A.V. Kotikov, A.I. Onishchenko and O.L. Veretin, Two-loop diagrams in non-relativistic $Q C D$ with elliptics: exact results, in preparation.

[113] A.V. Smirnov, FIESTA4: Optimized Feynman integral calculations with GPU support, Comput. Phys. Commun. 204 (2016) 189 [arXiv:1511.03614] [inSPIRE].

[114] J. Fleischer, A.V. Kotikov and O.L. Veretin, The differential equation method: Calculation of vertex type diagrams with one nonzero mass, Phys. Lett. B 417 (1998) 163 [hep-ph/9707492] [INSPIRE].

[115] J. Fleischer, A.V. Kotikov and O.L. Veretin, Analytic two loop results for selfenergy type and vertex type diagrams with one nonzero mass, Nucl. Phys. B 547 (1999) 343 [hep-ph/9808242] [INSPIRE].

[116] J. Fleischer, M.Y. Kalmykov and A.V. Kotikov, Two loop selfenergy master integrals on-shell, Phys. Lett. B 462 (1999) 169 [Erratum ibid. 467 (1999) 310] [hep-ph/9905249] [INSPIRE].

[117] A. Kotikov, J.H. Kühn and O. Veretin, Two-Loop Formfactors in Theories with Mass Gap and Z-Boson Production, Nucl. Phys. B 788 (2008) 47 [hep-ph/0703013] [InSPIRE].

[118] A.I. Davydychev and M.Y. Kalmykov, Massive Feynman diagrams and inverse binomial sums, Nucl. Phys. B 699 (2004) 3 [hep-th/0303162] [INSPIRE]. 